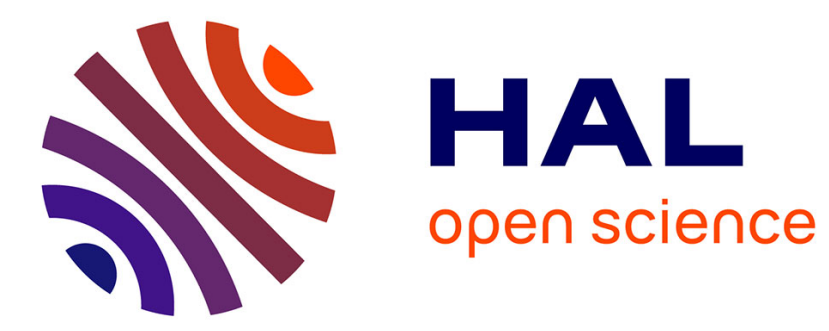

\title{
Behaviour of the Landau-Lifschitz equation in a ferromagnetic wire
}

David Sanchez

\section{To cite this version:}

David Sanchez. Behaviour of the Landau-Lifschitz equation in a ferromagnetic wire. Mathematical Methods in the Applied Sciences, 2009, 32 (2), pp.167 - 205. 10.1002/mma.1030 . hal-01872074

\section{HAL Id: hal-01872074 \\ https://hal.science/hal-01872074}

Submitted on 11 Sep 2018

HAL is a multi-disciplinary open access archive for the deposit and dissemination of scientific research documents, whether they are published or not. The documents may come from teaching and research institutions in France or abroad, or from public or private research centers.
L'archive ouverte pluridisciplinaire HAL, est destinée au dépôt et à la diffusion de documents scientifiques de niveau recherche, publiés ou non, émanant des établissements d'enseignement et de recherche français ou étrangers, des laboratoires publics ou privés. 


\title{
Behaviour of the Landau-Lifschitz equation in a ferromagnetic wire
}

\author{
DAVID SANCHEZ \\ Mathématiques Appliquées de Bordeaux, UMR 5466, Université Bordeaux 1, \\ 351 Cours de la Libération, 33405 Talence cedex, France.
}

\begin{abstract}
Following a suggestion from A. Thiaville and J. Miltat whose work and experiments are about ferromagnetic thin layers and nanowires we study in this paper the behaviour of the Landau-Lifschitz equation in a straight ferromagnetic wire. As the diameter of the domain and the exchange coefficient in the equation simultaneously tend to zero we perform an asymptotic expansion to precise the solution for well-prepared initial conditions and are lead to consider $2 D$ exterior problems.
\end{abstract}

Résumé. Suite à une suggestion d'A. Thiaville et J. Miltat dont les travaux et expériences portent sur les couches minces et les nanofils ferromagnétiques, nous étudions dans cet article le comportement de l'équation de Landau-Lifschitz dans un fil ferromagnétique rectiligne. Alors que le diamètre du domaine et le coefficient d'échange dans l'équation tendent simultanément vers zéro, nous effectuons un développement asymptotique, précisons la solution pour des données initiales bien préparées et sommes amenés à traiter des problèmes extérieurs en dimension 2 .

\section{Introduction}

Further to $[22,23]$ in which we study the behaviour of the solutions of the Landau-Lifschitz equation in a thin layer, A. Thiaville and J. Miltat whose work and experiments are about ferromagnetic material asked if models and asymptotic expansions could be proved in the case they were interested, i.e. ferromagnetic nanowires and particularly circular-based ones [25, 26].

We then consider here a ferromagnetic wire $\omega_{\varepsilon}$ whose diameter is of order $\varepsilon$ and placed in the vacuum. The ferromagnetic material is characterized by a spontaneous magnetization that is modelized by an unitary vector field $u^{\varepsilon}$ called the magnetic moment and defined on the domain $\omega_{\varepsilon}$ where the ferromagnetic material is confined. This magnetic moment links the magnetic field $\mathcal{H}\left(u^{\varepsilon}\right)$ and the magnetic induction $B$ by the relation $B=\mathcal{H}\left(u^{\varepsilon}\right)+\overline{u^{\varepsilon}}$ where $\overline{u^{\varepsilon}}$ is the extension of $u^{\varepsilon}$ by 0 outside $\omega_{\varepsilon}(c f[4])$. Its evolution is described by the Landau-Lifschitz equation (cf $[15])$ :

$$
\left\{\begin{array}{l}
\frac{\partial u^{\varepsilon}}{\partial t}=u^{\varepsilon} \wedge \mathcal{H}_{e f f}-u^{\varepsilon} \wedge\left(u^{\varepsilon} \wedge \mathcal{H}_{e f f}\right) \quad \text { in } \omega_{\varepsilon}, \\
\frac{\partial u^{\varepsilon}}{\partial \nu}=0 \quad \text { on } \partial \omega_{\varepsilon}, \\
u^{\varepsilon}(t=0, x)=u_{0}(x) \quad \text { in } \omega_{\varepsilon},
\end{array}\right.
$$

where $\nu$ denotes the outward unitary normal on $\partial \omega_{\varepsilon}$ and $\mathcal{H}_{e f f}=\varepsilon^{2} \Delta u^{\varepsilon}+\mathcal{H}\left(u^{\varepsilon}\right)+\varphi\left(u^{\varepsilon}\right)$ is the effective field composed of the exchange term $\varepsilon^{2} \Delta u^{\varepsilon}$ modelling the spin-like interactions in the ferromagnetic medium, $\varphi\left(u^{\varepsilon}\right)$ is an anisotropic field that takes into account the geometry of the

AMS Subject Classifications: 35Q60, 78M35, 34E20. 
material and $\mathcal{H}\left(u^{\varepsilon}\right)$ is the magnetic field. In the following we neglect the anisotropy field since it only induces more computations and has no mathematical influence on the results we obtain (at least for classical anisotropy fields deriving from quadratic anistropy energy). We moreover assume that we always are at the electromagnetic equilibrium:

$$
\begin{cases}\operatorname{div}\left(\mathcal{H}\left(u^{\varepsilon}\right)+\bar{u}^{\varepsilon}\right)=0 & \text { in } \mathbb{R}^{3}, \\ \operatorname{curl} \mathcal{H}\left(u^{\varepsilon}\right)=0 & \text { in } \mathbb{R}^{3},\end{cases}
$$

Many works take an interest in the behaviour of this magnetic moment: when the exchange coefficient is fixed and the domain $\omega_{\varepsilon}=\Omega$ does not depend on $\varepsilon$, Carbou and Fabrie prove in [7] the local existence of a strong $\mathbb{H}^{2}(\Omega)$ solution of (1.1). When the exchange coefficient $\varepsilon^{2}$ goes to zero and $\omega_{\varepsilon}=\Omega$, Carbou, Fabrie and Guès prove in [9] that the time of existence goes to infinity when $\varepsilon$ goes to zero for an initial data in $\mathbb{H}^{5}(\Omega)$ (instead of $\mathbb{H}^{2}(\Omega)$ ) by proving that a boundary layer whose characteristic thickness is of order $\varepsilon$ appears. Moreover they obtain that the solution $u^{\varepsilon}$ tends to the solution of the hyperbolic system formally obtained by taking $\varepsilon=0$ in (1.1). Their theorem however does not give a first order asymptotic expansion of the solution. These results are established in the case where the domain $\omega_{\varepsilon}$ does not depend on $\varepsilon$. Interesting phenomena happen when the ferromagnetic material lies in small domains such as thin layers and nanowires. In these cases we expect a much simpler behaviour and we need to justify the models in use. In the case of thin layers, Carbou studies in [6] by means of energy estimates the limit of the magnetic field $\mathcal{H}\left(u^{\varepsilon}\right)$ when the thickness $\varepsilon$ of the domain goes to 0 and the exchange coefficient is fixed. In a previous work [22] we consider the case of a flat periodic thin layer in the vacuum when the exchange coefficient is $\varepsilon^{2}$ and the thickness of the layer is $\varepsilon$. In this critical case where the thickness of the thin layer and of the boundary layer are of the same order we justify a first-order asymptotic expansion of the magnetic moment and of the magnetic field for small values of $\varepsilon$ and find again the classical physical description of such a case. In [23] we extend these results to a non-flat geometry by considering a thin layer of ferromagnetic material spread on a perfect conductor.

In this paper we consider the other kind of studied domain, i.e. the case of a periodic straight ferromagnetic wire placed in the the case of a straight ferromagnetic wire placed in the vacuum. We want to establish and justify the passage from the $3 D$ case to the $1 D$ case. As for the case of thin layers, we expect to have an interaction between the boundary layers that arise from the exchange coefficient and whose size is of order $\varepsilon$, and the small dimension of the domain, also of order $\varepsilon$. A rescaling shows that there is no boundary layer in the rescaled domain and that the Landau-Lifschitz equation becomes anisotropic. The small size of the domain also influences the magnetostatic equations as we will see it later and leads us to work with $\varepsilon$-dependent domains and to precise the dependence on $\varepsilon$ of the Sobolev embeddings.

Remark 1.1. We consider in the following a periodic straight wire pointing at the direction $e_{3}$. The assumption of the periodic wire allows us to forget the formation of a boundary layer at the ends of the wire. The domain we then consider writes $\omega_{\varepsilon}=\varepsilon \Omega \times I_{1}$ where $\Omega$ is an open bounded regular star-shaped set in $\mathbb{R}^{2}$ and $I_{1}=(0,1)$ is the periodic domain. These results remain valid in $\varepsilon \Omega \times \mathbb{R}$ if we use the Fourier transform.

In the following we denote $\mathbb{H}_{p}^{l}\left(I_{1}\right)$ the subspace of $\mathbb{H}^{l}\left(I_{1}\right)$ whose functions are periodic on $I_{1}$, and by $\mathbb{L}_{p}^{2}\left(I_{1}\right)$ the closure of $\mathbb{H}_{p}^{1}\left(I_{1}\right)$ in $\mathbb{L}^{2}\left(I_{1}\right)$. The spaces $\mathbb{H}^{k}(V) \otimes \mathbb{H}^{p}\left(I_{1}\right)$ are the classical tensor spaces endowed with the norm $\|u\|=\|\| u(\cdot, z)\left\|_{\mathbb{H}^{k}(V)}\right\|_{H^{p}\left(I_{1}\right)}$. 
We also use the following decompositions : for $V \in \mathbb{R}^{3}$, we let $V_{l}=\left(V, e_{3}\right)$ the longitudinal component of $V$ and $V_{\tau}=V-V_{l} e_{3}$ the transversal component. We let $\nabla_{\tau}$ the transversal component of $\nabla$, i.e. $\nabla_{\tau}=\left(\partial_{x_{1}}, \partial_{x_{2}}, 0\right)^{t}$ and

$$
\operatorname{div}_{\tau}=\left(\nabla_{\tau}, \cdot\right), \quad \operatorname{curl}_{\tau}=\left(\nabla_{\tau} \wedge \cdot\right), \quad \Delta_{\tau}=\operatorname{div}_{\tau} \nabla_{\tau}
$$

In order to perform the asymptotic expansion and obtain the profiles we work on a rescaled form of the Landau-Lifschitz equation. We obtain the following result:

Theorem 1.1. Let $u_{0}^{\varepsilon}$ an initial data satisfying $\frac{\partial u_{0}^{\varepsilon}}{\partial \nu}=0$ on $\partial \omega_{\varepsilon}$ and which can be written in the form:

$$
u_{0}^{\varepsilon}(x)=U_{0}^{0}\left(\frac{\sigma}{\varepsilon}, z\right), \quad \forall x=(\varepsilon \sigma, z) \in \omega_{\varepsilon},
$$

where $U_{0}^{0} \in \mathbb{H}^{6}(\Omega) \otimes \mathbb{H}_{p}^{7}\left(I_{1}\right)$ and $\left|U_{0}^{0}\right| \equiv 1$. There exists $T_{0}>0$ independent from $\varepsilon$ and there exists two profiles $U^{0}, U^{1}$ fulfilling

- $U^{0} \in \bigcap_{k=0}^{3} \mathbb{W}^{k, \infty}\left(0, T_{0} ; \mathbb{H}^{6-2 k}(\Omega) \otimes \mathbb{H}_{p}^{7}\left(I_{1}\right)\right) \cap \mathbb{H}^{k}\left(0, T_{0} ; \mathbb{H}^{7-2 k}(\Omega) \otimes \mathbb{H}_{p}^{7}\left(I_{1}\right)\right)$ is solution of:

$$
\left\{\begin{array}{l}
\frac{\partial U^{0}}{\partial t}-\Delta_{\tau} U^{0}=\left|\nabla_{\tau} U^{0}\right|^{2} U^{0}+U^{0} \wedge \Delta_{\tau} U^{0}+U^{0} \wedge H^{0}-U^{0} \wedge\left(U^{0} \wedge H^{0}\right) \quad \text { in } \Omega \times I_{1}, \\
\frac{\partial U^{0}}{\partial \nu}=0 \quad \text { on } \partial \Omega \times I_{1}, \\
U^{0}(t=0)=U_{0}^{0} \quad \text { in } \Omega \times I_{1}, \\
H^{0}=\mathcal{H}\left(U^{0}\right) \quad \text { in } \Omega \times I_{1},
\end{array}\right.
$$

where $-H^{0}$ is the projection of $U^{0}$ on the transversal gradient field,

$$
\text { - } U^{1} \in \bigcap_{k=0}^{2}\left(\mathbb{W}^{k, \infty}\left(0, T_{0} ; \mathbb{H}^{5-2 k}(\Omega) \otimes \mathbb{H}_{p}^{6}\left(\mathbb{R}_{z}\right)\right) \cap \mathbb{H}^{k}\left(0, T_{0} ; \mathbb{H}^{6-2 k}(\Omega) \otimes \mathbb{H}_{p}^{6}\left(\mathbb{R}_{z}\right)\right)\right) \text {, }
$$

such that the regular solution of Eq. (1.1)-(1.2) exists on $\left[0, T_{0}\right]$ and has an asymptotic expansion in the form:

$$
u^{\varepsilon}(t, x)=U^{0}\left(t, \frac{\sigma}{\varepsilon}, z\right)+\varepsilon U^{1}\left(t, \frac{\sigma}{\varepsilon}, z\right)+\varepsilon^{2} r^{\varepsilon}(t, x), \quad x=(\varepsilon \sigma, z) \in \omega_{\varepsilon}, t \in\left[0, T_{0}\right],
$$

where for all $2 \leq p \leq+\infty, r^{\varepsilon} \in \mathbb{L}^{\infty}\left(0, T_{0} ; \mathbb{L}^{p}\left(\omega_{\varepsilon}\right)\right)$ and :

$$
\sup _{t \in\left[0, T_{0}\right]}\left\|r^{\varepsilon}\right\|_{\mathbb{L}^{p}\left(\omega_{\varepsilon}\right)}=\mathcal{O}\left(\varepsilon^{2 / p}\right) .
$$

In the case where the section of the wire is a disk we enhance this result in the following way:

Theorem 1.2. Let $\Omega=D(0,1)$ the unitary disk and $\omega_{\varepsilon}=\varepsilon \Omega \times I_{1}$. Let $u_{0}^{\varepsilon}$ an initial data which can be written in the form:

$$
u_{0}^{\varepsilon}(x)=U_{0}^{0}(z), \quad \forall x=(\varepsilon \sigma, z) \in \omega_{\varepsilon},
$$

where $U_{0}^{0} \in \mathbb{H}_{p}^{7}\left(I_{1}\right)$ is invariant in the section of the wire and $\left|U_{0}^{0}\right| \equiv 1$. If $T^{\varepsilon}$ is the maximum time of existence of the regular solution of (1.1)-(1.2) then $\lim _{\varepsilon \rightarrow 0} T^{\varepsilon}=+\infty$ and for all $0<$ $T<T^{\varepsilon}$ there exists two profiles $U^{0}, U^{1}$ fulfilling 
- $U^{0} \in \mathcal{C}^{\infty}\left(\mathbb{R}_{t}^{+} ; \mathbb{H}_{p}^{7}(\Omega)\right)$ is solution of the following ordinary differential equation:

$$
\left\{\begin{array}{l}
\frac{\partial U^{0}}{\partial t}=U^{0} \wedge H^{0}-U^{0} \wedge\left(U^{0} \wedge H^{0}\right) \quad \text { in } I_{1}, \\
U^{0}(t=0)=U_{0}^{0} \quad \text { in } I_{1},
\end{array}\right.
$$

where $H^{0}=-\frac{1}{2} U_{\tau}^{0}$ is a local operator.

- $U^{1} \in \bigcap_{k=0}^{2}\left(\mathbb{W}^{k, \infty}\left(0, T ; \mathbb{H}^{5-2 k}(\Omega) \otimes \mathbb{H}_{p}^{6}\left(\mathbb{R}_{z}\right)\right) \cap \mathbb{H}^{k}\left(0, T ; \mathbb{H}^{6-2 k}(\Omega) \otimes \mathbb{H}_{p}^{6}\left(\mathbb{R}_{z}\right)\right)\right), \forall T>0$

such that $u^{\varepsilon}$ has an asymptotic expansion in the form:

$$
u^{\varepsilon}(t, x)=U^{0}(t, z)+\varepsilon U^{1}\left(t, \frac{\sigma}{\varepsilon}, z\right)+\varepsilon^{2} r^{\varepsilon}(t, x), \quad x=(\varepsilon \sigma, z) \in \omega_{\varepsilon}, t \in\left[0, T_{0}\right],
$$

where for all $2 \leq p \leq+\infty, r^{\varepsilon} \in \mathbb{L}^{\infty}\left(0, T^{\varepsilon} ; \mathbb{L}^{p}\left(\omega_{\varepsilon}\right)\right)$ and :

$$
\sup _{t \in\left[0, T^{\varepsilon}\right]}\left\|r^{\varepsilon}\right\|_{\mathbb{L}^{p}\left(\omega_{\varepsilon}\right)}=\mathcal{O}\left(\varepsilon^{2 / p}\right) .
$$

\section{Remark 1.2.}

- In the case where the section of the wire is a disk we prove that the magnetic field is a local operator at the order $\varepsilon^{0}$. We then obtain a monodimensional model for the wire which is an ordinary differential equation with a $1 D$ parameter.

- The limit model we obtain has the expected behaviour and matches the physical observations.

This paper is organized in the following way. In the first part we formally build the asymptotic expansion of $u^{\varepsilon}$ and we prove the local existence and the regularity of its terms. Consequently we study the operator $\mathcal{H}$ and are lead to obtain regular solutions to $2 D$ exterior problem for which we introduce weighted Sobolev spaces. In the second part we introduce anisotropic and $\varepsilon$-dependent norms adapted to the size of the domain and we give all the $\varepsilon$-dependent Sobolev embeddings and energy estimates in the space $\omega_{\varepsilon}$ that we need in the last parts. The equation satisfied by the remainder terms of $u^{\varepsilon}$ and $H^{\varepsilon}$ are explained in the third part while the fourth contains the energy estimates that conclude the proof of theorem 1.1.

\section{Formal asymptotic expansion}

As the diameter of the wire is of order $\varepsilon$ we want to get rid of the $\varepsilon$ parameter and explain the behaviour in the small domain. If we only rescale the equations inside the ferromagnetic wire, we are lead to solve the magnetostatic equations in a non simply connected exterior domain which depends on $\varepsilon$. We instead perform a rescaling in the whole space, thus transforming the magnetostatic equations in the exterior domain.

We will now search an asymptotic expansion of $u^{\varepsilon}$ and $H^{\varepsilon}=\mathcal{H}\left(u^{\varepsilon}\right)$ in the form:

- in $\omega_{\varepsilon}$,

$$
\begin{aligned}
& u^{\varepsilon}(t, x)=U^{0}\left(t, \frac{\sigma}{\varepsilon}, z\right)+\varepsilon U^{1}\left(t, \frac{\sigma}{\varepsilon}, z\right)+\ldots \\
& H^{\varepsilon}(t, x)=H^{0}\left(t, \frac{\sigma}{\varepsilon}, z\right)+\varepsilon H^{1}\left(t, \frac{\sigma}{\varepsilon}, z\right)+\ldots
\end{aligned}
$$


- in $\left(\mathbb{R}^{2} \times I_{1}\right) \backslash \omega_{\varepsilon}$,

$$
H^{\varepsilon}(t, x)=H^{0}\left(t, \frac{\sigma}{\varepsilon}, z\right)+\varepsilon H^{1}\left(t, \frac{\sigma}{\varepsilon}, z\right)+\ldots
$$

where $x=(\sigma, z) \in \mathbb{R}^{2} \times I_{1}$.

Let us perform some preliminary calculus on the curl and divergence operator. If we have $V(x)=\sum_{i \geq 0} V^{i}\left(\frac{\sigma}{\varepsilon}, z\right)$

$$
\begin{aligned}
& \operatorname{curl} V=\frac{1}{\varepsilon} \operatorname{curl}_{\tau} V^{0}+\sum_{i \geq 0} \varepsilon^{i}\left(\frac{\partial}{\partial z}\left(e_{3} \wedge V^{i}\right)+\operatorname{curl}_{\tau} V^{i+1}\right) \\
& \operatorname{div} V=\frac{1}{\varepsilon} \operatorname{div}_{\tau} V^{0}+\sum_{i \geq 0} \varepsilon^{i}\left(\frac{\partial}{\partial z} V_{l}^{i}+\operatorname{div}_{\tau} V^{i+1}\right)
\end{aligned}
$$

where $\operatorname{curl}_{\tau} V=\nabla_{\tau} \wedge V=\left(\nabla_{\tau} V_{3}\right)^{\perp}+\left(\begin{array}{c}0 \\ 0 \\ \operatorname{curl}_{2 D} V_{T}\end{array}\right)$, and $A^{\perp}=A \wedge e_{3}$.

\subsection{Equations of the profiles}

As it is proved in [7], the equation (1.1) is equivalent for regular enough solutions to:

$$
\frac{\partial u^{\varepsilon}}{\partial t}-\varepsilon^{2} \Delta u^{\varepsilon}=\varepsilon^{2}\left|\nabla u^{\varepsilon}\right|^{2} u^{\varepsilon}+\varepsilon^{2} u^{\varepsilon} \wedge \Delta u^{\varepsilon}+u^{\varepsilon} \wedge \mathcal{H}\left(u^{\varepsilon}\right)-u^{\varepsilon} \wedge\left(u^{\varepsilon} \wedge \mathcal{H}\left(u^{\varepsilon}\right)\right) .
$$

Since this equation isolates the dissipative term we will use it in the following. We then substitute to $u^{\varepsilon}, \mathcal{H}\left(u^{\varepsilon}\right)$ their asymptotic expansions (2.1) and (2.2) in the equations (1.2) and (2.4) to obtain the expression fulfilled by the profiles for $(\sigma, z)$ in and outside $\omega_{\varepsilon}$.

As the equations (1.2) are linear, each $H^{i}$ fulfills outside $\omega_{\varepsilon}$ :

$$
\begin{cases}\operatorname{div}_{\tau} H_{\tau}^{i}+\partial_{z} H_{l}^{i-1}=0 & \text { in } \Omega^{\prime} \times I_{1} \\ \operatorname{curl}_{2 D} H_{\tau}^{i}=0 & \text { in } \Omega^{\prime} \times I_{1} \\ \nabla_{\tau} H_{l}^{i}=\partial_{z} H_{\tau}^{i-1} & \text { in } \Omega^{\prime} \times I_{1}\end{cases}
$$

where $\Omega^{\prime}=\mathbb{R}^{2} \backslash \Omega$. As we will also determine the equations fulfilled by $H^{i}$ in $\Omega \times I_{1}$, we need some transmission conditions on the boundary $\partial \Omega \times I_{1}$ that we deduce from Eq. (1.2):

$$
\begin{cases}{\left[H^{i} \wedge \nu\right]=0} & \text { on } \partial \omega_{\varepsilon} \\ {\left[\left(H^{i}+\bar{U}^{i}\right) \cdot \nu\right]=0} & \text { on } \partial \omega_{\varepsilon}\end{cases}
$$

where $[f]$ denotes the jump of $f$ at the interface $\partial \omega_{\varepsilon}$. Changing the notation it also writes:

$$
\begin{cases}{\left[H_{l}^{i}\right]=0} & \text { on } \partial \Omega \times I_{1}, \\ {\left[H_{\tau}^{i} \wedge \nu\right]=0} & \text { on } \partial \Omega \times I_{1}, \\ {\left[\left(H_{\tau}^{i}+\bar{U}_{\tau}^{i}\right) \cdot \nu\right]=0} & \text { on } \partial \Omega \times I_{1} .\end{cases}
$$


The Neumann condition on $u^{\varepsilon}$ also becomes $\frac{\partial U^{i}}{\partial \nu}=0$ for all $x=(\sigma, z) \in \partial \omega_{\varepsilon}=(\varepsilon \partial \Omega) \times I_{1}$ and $i \in \mathbb{N}$.

Terms in $\varepsilon^{-1}:$ in $\Omega \times I_{1}$

$$
\left\{\begin{array}{l}
\operatorname{div}_{\tau}\left(H_{\tau}^{0}+U_{\tau}^{0}\right)=0, \\
\operatorname{curl}_{2 D} H_{\tau}^{0}=0 \\
\nabla_{\tau} H_{l}^{0}=0
\end{array}\right.
$$

From the last equation and the first transmission condition in (2.6) we deduce that $H_{l}^{0} \equiv$ $H_{l}^{0}(t, z)$. As we do not impose an external magnetic field and as we are looking for a solution in $\mathbb{L}^{2}\left(\mathbb{R}^{3}\right)$, we set $H_{l}^{0} \equiv 0$. The other components of $H^{0}$, i.e. $H_{\tau}^{0}$, fulfills then

$$
\begin{cases}H^{0} \in \mathbb{L}^{2}\left(\mathbb{R}^{2} \times I_{1}\right), & \\ \operatorname{div}_{\tau}\left(H_{\tau}^{0}+U_{\tau}^{0}\right)=0 & \text { on } \mathbb{R}^{2} \times I_{1}, \\ \operatorname{curl}_{2 D} H_{\tau}^{0}=0 & \text { on } \mathbb{R}^{2} \times I_{1} .\end{cases}
$$

As we do not impose an external magnetic field, we obtain that $-H^{0}$ is the projection of $U_{\tau}^{0}$ and then of $U^{0}$ - on the vector field of transversal gradients.

Terms in $\varepsilon^{0}:$ in $\Omega \times I_{1}$,

$$
\begin{aligned}
\frac{\partial U^{0}}{\partial t}-\Delta_{\tau} U^{0}= & \left|\nabla_{\tau} U^{0}\right|^{2} U^{0}+U^{0} \wedge \Delta_{\tau} U^{0}+U^{0} \wedge H^{0}-U^{0} \wedge\left(U^{0} \wedge H^{0}\right) . \\
& \left\{\begin{array}{l}
\operatorname{div}_{\tau}\left(H_{\tau}^{1}+U_{\tau}^{1}\right)+\partial_{z}\left(H_{l}^{0}+U_{l}^{0}\right)=0 \\
\operatorname{curl}_{2 D} H_{\tau}^{1}=0 \\
\nabla_{\tau} H_{l}^{1}=\partial_{z} H_{\tau}^{0}
\end{array}\right.
\end{aligned}
$$

Terms in $\varepsilon:$ in $\Omega \times I_{1}$,

$$
\begin{aligned}
\frac{\partial U^{1}}{\partial t}-\Delta_{\tau} U^{1}= & \left|\nabla_{\tau} U^{0}\right|^{2} U^{1}+2\left(\nabla_{\tau} U^{0} \cdot \nabla_{\tau} U^{1}\right) U^{0}+U^{0} \wedge \Delta_{\tau} U^{1}+U^{1} \wedge \Delta_{\tau} U^{0} \\
& +U^{0} \wedge H^{1}+U^{1} \wedge H^{0}-U^{0} \wedge\left(U^{0} \wedge H^{1}\right)-U^{0} \wedge\left(U^{1} \wedge H^{0}\right) \\
& -U^{1} \wedge\left(U^{0} \wedge H^{0}\right) . \\
& \left\{\begin{array}{l}
\operatorname{div}_{\tau}\left(H_{\tau}^{2}+U_{\tau}^{2}\right)+\partial_{z}\left(H_{l}^{1}+U_{l}^{1}\right)=0 \\
\operatorname{curl}_{2 D} H_{\tau}^{2}=0 \\
\nabla_{\tau} H_{l}^{2}=\partial_{z} H_{\tau}^{1} .
\end{array}\right.
\end{aligned}
$$

Terms in $\varepsilon^{2}$ : in $\Omega \times I_{1}$,

$$
\begin{aligned}
\frac{\partial U^{2}}{\partial t}-\Delta_{\tau} U^{2}= & \left|\nabla_{\tau} U^{0}\right|^{2} U^{2}+2\left(\nabla_{\tau} U^{0} \cdot \nabla_{\tau} U^{2}\right) U^{0}+U^{0} \wedge \Delta_{\tau} U^{2}+U^{2} \wedge \Delta_{\tau} U^{0} \\
& +U^{0} \wedge H^{2}+U^{2} \wedge H^{0}-U^{0} \wedge\left(U^{0} \wedge H^{2}\right)-U^{0} \wedge\left(U^{2} \wedge H^{0}\right) \\
& -U^{2} \wedge\left(U^{0} \wedge H^{0}\right) \\
& +U_{z z}^{0}+\left|U_{z}^{0}\right|^{2} U^{0}+U^{0} \wedge U_{z z}^{0}+U^{1} \wedge \Delta_{\tau} U^{1}+\left|\nabla_{\tau} U^{1}\right|^{2} U^{0} \\
& +2\left(\nabla_{\tau} U^{0} \cdot \nabla_{\tau} U^{1}\right) U^{1}+U^{1} \wedge H^{1}-U^{0} \wedge\left(U^{1} \wedge H^{1}\right) \\
& -U^{1} \wedge\left(U^{0} \wedge H^{1}\right)-U^{1} \wedge\left(U^{1} \wedge H^{0}\right)
\end{aligned}
$$




$$
\left\{\begin{array}{l}
\operatorname{div}_{\tau}\left(H_{\tau}^{3}+U_{\tau}^{3}\right)+\partial_{z}\left(H_{l}^{2}+U_{l}^{2}\right)=0 \\
\operatorname{curl}_{2 D} H_{\tau}^{3}=0 \\
\nabla_{\tau} H_{l}^{3}=\partial_{z} H_{\tau}^{2}
\end{array}\right.
$$

Termes d'ordre $\varepsilon^{3}$ : dans $\Omega \times I_{1}$

$$
\begin{aligned}
\frac{\partial U^{3}}{\partial t}-\Delta_{\tau} U^{3}= & \left|\nabla_{\tau} U^{0}\right|^{2} U^{3}+2\left(\nabla_{\tau} U^{0} \cdot \nabla_{\tau} U^{3}\right) U^{0}+U^{0} \wedge \Delta_{\tau} U^{3}+U^{3} \wedge \Delta_{\tau} U^{0} \\
& +U^{0} \wedge H^{3}+U^{3} \wedge H^{0}-U^{0} \wedge\left(U^{0} \wedge H^{3}\right)-U^{0} \wedge\left(U^{3} \wedge H^{0}\right) \\
& -U^{3} \wedge\left(U^{0} \wedge H^{0}\right) \\
& +\partial_{z}^{2} U^{1}+\left|\nabla_{\tau} U^{1}\right|^{2} U^{0}+2\left(\nabla_{\tau} U^{0} \cdot \nabla_{\tau} U^{1}\right) U^{2}+2\left(\nabla_{\tau} U^{0} \cdot \nabla_{\tau} U^{2}\right) U^{1} \\
& +2\left(\nabla_{\tau} U^{1} \cdot \nabla_{\tau} U^{2}\right) U^{0}+\left|\partial_{z} U^{0}\right|^{2} U^{1}+2\left(\partial_{z} U^{0} \cdot \partial_{z} U^{1}\right) U^{0} \\
& +U^{1} \wedge \partial_{z}^{2} U^{0}+U^{0} \wedge \partial_{z}^{2} U^{1}+U^{1} \wedge \Delta_{\tau} U^{2}+U^{2} \wedge \Delta_{\tau} U^{1} \\
& +U^{1} \wedge H^{2}+U^{2} \wedge H^{1}-U^{0} \wedge\left(U^{1} \wedge H^{2}\right)-U^{0} \wedge\left(U^{2} \wedge H^{1}\right) \\
& -U^{1} \wedge\left(U^{0} \wedge H^{2}\right)-U^{1} \wedge\left(U^{2} \wedge H^{0}\right)-U^{2} \wedge\left(U^{0} \wedge H^{1}\right) \\
& -U^{1} \wedge\left(U^{2} \wedge H^{0}\right)-U^{1} \wedge\left(U^{1} \wedge H^{1}\right) .
\end{aligned}
$$

\subsection{Existence of the asymptotic expansion}

\subsubsection{Behaviour of $H$}

In the following we have to solve the exterior problem:

$$
\left\{\begin{array}{l}
\operatorname{div}(H+\bar{u})=f \quad \text { in } \mathbb{R}^{2}, \\
\operatorname{curl} H=0 \quad \text { in } \mathbb{R}^{2},
\end{array}\right.
$$

where $u$ is only defined in a regular open bounded subset $\Omega$ of $\mathbb{R}^{2}$ and $\bar{u}$ is the extension of $u$ by 0 outside $\Omega$.

Following the work of M.N. Le Roux [16] on the $2 D$ exterior problem we introduce weighted Sobolev spaces adapted to the solving of the problem:

Definition 2.1. Let $\rho=\left(1+|x|^{2}\right)^{1 / 2}$. We define the weighted Sobolev spaces $\mathbf{W}_{\alpha}^{l}\left(\mathbb{R}^{2}\right)$ by:

$$
\begin{aligned}
& \mathbf{W}_{1}^{1}\left(\mathbb{R}^{2}\right)=\left\{u \in \mathcal{D}^{\prime}\left(\mathbb{R}^{2}\right), \frac{1}{\rho(1+\ln \rho)} u \in \mathbb{L}^{2}\left(\mathbb{R}^{2}\right), \nabla u \in \mathbb{L}^{2}\left(\mathbb{R}^{2}\right)\right\}, \\
& \mathbf{W}_{2}^{2}\left(\mathbb{R}^{2}\right)=\left\{u \in \mathcal{D}^{\prime}\left(\mathbb{R}^{2}\right), \frac{1}{\rho^{2}(1+\ln \rho)} u \in \mathbb{L}^{2}\left(\mathbb{R}^{2}\right), \frac{1}{\rho(1+\ln \rho)} \nabla u \in \mathbb{L}^{2}\left(\mathbb{R}^{2}\right), D^{2} u \in \mathbb{L}^{2}\left(\mathbb{R}^{2}\right)\right\}, \\
& \mathbf{W}_{\alpha}^{l}\left(\mathbb{R}^{2}\right)=\left\{u \in \mathcal{D}^{\prime}\left(\mathbb{R}^{2}\right), \frac{1}{\rho^{\alpha-|\beta|}(1+\ln \rho)} \partial^{\beta} u \in \mathbb{L}^{2}\left(\mathbb{R}^{2}\right),|\beta| \leq l\right\},
\end{aligned}
$$

where $l \in \mathbb{N}$ and $\alpha>l$.

To solve the exterior problem, we need equivalent norms on the previous spaces:

Proposition 2.1. There exists a constant $C$ such that, for all $u \in \mathbf{W}_{1}^{1}\left(\mathbb{R}^{2}\right)$, we have:

$$
\left\|\frac{u}{\rho(1+\ln \rho)}\right\|_{\mathbb{L}^{2}\left(\mathbb{R}^{2}\right)} \leq C\|\nabla u\|_{\mathbb{L}^{2}\left(\mathbb{R}^{2}\right)} .
$$

By the same way, for all $u \in \mathbf{W}_{\alpha}^{1}\left(\mathbb{R}^{2}\right), \alpha>1$ we have:

$$
\left\|\frac{u}{\rho^{\alpha}(1+\ln \rho)}\right\|_{\mathbb{L}^{2}\left(\mathbb{R}^{2}\right)} \leq C\left\|\frac{1}{\rho^{\alpha-1}(1+\ln \rho)} \nabla u\right\|_{\mathbb{L}^{2}\left(\mathbb{R}^{2}\right)} .
$$


Proof. we use a generalized Hardy inequality proved by Bolley and Camus in [2]. See also Nédélec [20].

Proposition 2.2. The $2 D$ magnetostatic equations

$$
\begin{cases}\operatorname{div}(\mathcal{H}(u)+\bar{u})=0 & \text { in } \mathbb{R}^{2}, \\ \operatorname{curl}_{2 D} \mathcal{H}(u)=0 & \text { in } \mathbb{R}^{2}, \\ {[(\mathcal{H}(u)+\bar{u}) \cdot \nu]=0} & \text { on } \partial \Omega\end{cases}
$$

where $u \in \mathbb{H}^{n}(\Omega), n \in \mathbb{N}^{*}$, has an unique solution $\mathcal{H}(u) \in \mathbb{H}^{s}\left(\mathbb{R}^{2}\right)$. Moreover there exists $\varphi \in \mathbb{W}_{1}^{1}\left(\mathbb{R}^{2}\right)$ such that $\mathcal{H}(u)=-\nabla \varphi$ and

$$
\|\varphi\|_{\mathbb{W}_{1}^{1}\left(\mathbb{R}^{2}\right)} \leq C\|u\|_{\mathbb{L}^{2}(\Omega)}, \quad\|\mathcal{H}(u)\|_{\mathbb{H}^{n}\left(\mathbb{R}^{2}\right)} \leq C\|u\|_{\mathbb{H}^{n}\left(\mathbb{R}^{2}\right)} .
$$

Proof. We combine ideas taken from $[7,9,14]$ for the existence and regularity of the solutions with the space $\mathbf{W}_{1}^{1}\left(\mathbb{R}^{2}\right)$ [16]. We follow the same steps as in $[7,9]$ :

Step 1: As curl $\mathcal{H}(u)=0$ in $\mathbb{R}^{2}$ there exists a regular function $\varphi$ such that $\mathcal{H}(u)=-\nabla \varphi$ and $[\varphi]_{\mid \partial \Omega}=0$ where $[f]$ denotes the jump of $f$ at the interface $\partial \Omega . \varphi$ fulfills:

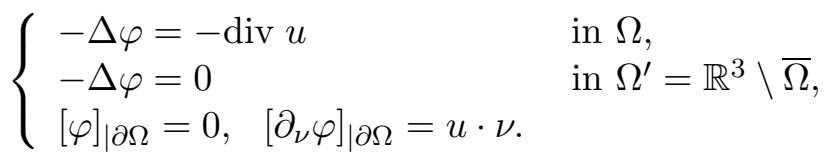

Step 2: Let $\Omega_{1}$ an open bounded subset of $\mathbb{R}^{2}$ containing $\Omega$. By surjectivity of the trace operator, there exists $\psi_{1} \in \mathbb{H}^{n+1}\left(\Omega_{1} \backslash \Omega\right)$ such that

$$
\left\|\psi_{1}\right\|_{\mathbb{H}^{n+1}\left(\Omega_{1} \backslash \Omega\right)} \leq C\|u\|_{\mathbb{H}^{n}(\Omega)},
$$

and

$$
\psi_{1 \mid \partial \Omega}=0, \psi_{1 \mid \partial \Omega_{1}^{\prime}}=0, \partial_{\nu} \psi_{1 \mid \partial \Omega_{1}^{\prime}}=0, \partial_{\nu} \psi_{1 \mid \partial \Omega}=u \cdot \nu,
$$

where $\Omega_{1}^{\prime}=\mathbb{R}^{2} \backslash \Omega_{1}$. We let $\varphi=\varphi_{1}+\psi_{1}$ and we have to solve:

$$
\begin{cases}-\Delta \varphi_{1}=-\operatorname{div} u & \text { in } \Omega \\ -\Delta \varphi_{1}=\Delta \psi_{1} & \text { in } \Omega_{1} \backslash \Omega \\ -\Delta \varphi_{1}=0 & \text { in } \Omega_{1}^{\prime}, \\ {\left[\varphi_{1}\right]_{\mid \partial \Omega_{i}}=0,\left[\partial_{\nu} \varphi_{1}\right]_{\mid \partial \Omega_{i}}=0, i=0,1,} & \end{cases}
$$

where $\Omega_{0}=\Omega$. We let:

$$
f_{1}=-\mathbf{1}_{\Omega} \operatorname{div} u, \quad f_{2}=\mathbf{1}_{\Omega_{2} \backslash \Omega} \Delta \psi_{1}, \quad F=f_{1}+f_{2} .
$$

We are lead to solve $-\Delta \varphi_{1}=F$ in $\mathbb{R}^{2}$ where $F$ is regular and with compact support. Thanks to an integration by parts, Prop. 2.1 and the Lax-Milgram theorem we obtain the existence and uniqueness of $\varphi_{1} \in \mathbf{W}_{1}^{1}\left(\mathbb{R}^{2}\right)$ hence the existence of $\varphi \in \mathbf{W}_{1}^{1}\left(\mathbb{R}^{2}\right)$ and of $H \in \mathbb{L}^{2}\left(\mathbb{R}^{2}\right)$. Moreover we have:

$$
\|\varphi\|_{\mathbf{W}_{1}^{1}\left(\mathbb{R}^{2}\right)} \leq C\|u\|_{\mathbb{L}^{2}\left(\mathbb{R}^{2}\right)}
$$

Classical regularity results on the solution of the Laplace operator ( $c f[3])$ improve the estimates:

$$
\|\nabla \varphi\|_{\mathbb{H}^{1}\left(\mathbb{R}^{2}\right)}=\|\mathcal{H}(u)\|_{\mathbb{H}^{1}\left(\mathbb{R}^{2}\right)} \leq C\|u\|_{\mathbb{L}^{2}\left(\mathbb{R}^{2}\right)} .
$$

Step 3: By following [7, 14], Step 2 and with the linearity of Eq. (2.15), we get the regularity result. 
Proposition 2.3. The $2 D$ exterior problem

$$
\begin{cases}\operatorname{div} H=\mathbf{1}_{\Omega} f & \text { in } \mathbb{R}^{2} \\ \operatorname{curl}_{2 D} H=0 & \text { in } \mathbb{R}^{2} \\ {[H \cdot \nu]=0, \quad[H \wedge \nu]=0} & \text { on } \partial \Omega\end{cases}
$$

where $f \in \mathbb{H}^{s}(\Omega), s \in \mathbb{N}$, has a unique solution $H \in \mathbb{H}^{s}\left(\mathbb{R}^{2}\right)$ with $H=-\nabla \varphi$ with $\varphi \in \mathbf{W}_{1}^{1}\left(\mathbb{R}^{2}\right)$. Moreover, we have:

$$
\|H\|_{\mathbb{H}^{s+1}\left(\mathbb{R}^{2}\right)} \leq C\|f\|_{\mathbb{H}^{s}(\Omega)}, \quad\|\varphi\|_{\mathbf{W}_{1}^{1}\left(\mathbb{R}^{2}\right)} \leq C\|f\|_{\mathbb{L}^{2}\left(\mathbb{R}^{2}\right)}
$$

Proof. We combine ideas taken from [7, 9, 14] for the existence and regularity of the solutions with the space $\mathbf{W}_{1}^{1}\left(\mathbb{R}^{2}\right)[16]$. The proof follows the same steps as in Lemma 2.15 except the integration by parts in Step 2.

In the following, we are also lead to solve:

$$
\begin{cases}\operatorname{div} H=f & \text { in } \mathbb{R}^{2} \\ \operatorname{curl}_{2 D} H=0 & \text { in } \mathbb{R}^{2} \\ {[H \cdot \nu]=0, \quad[H \wedge \nu]=0} & \text { on } \partial \Omega\end{cases}
$$

where $f$ is such that $-\nabla f$ is either solution of Eq. (2.15), either solution of Eq. (2.16). The first problem also writes:

$$
\left\{\begin{array}{l}
\operatorname{div}(\Delta H+\bar{u})=0 \quad \text { in } \mathbb{R}^{2} \\
\operatorname{curl}_{2 D} H=0 \quad \text { in } \mathbb{R}^{2} \\
{[H \cdot \nu]=0, \quad[(\Delta H+\bar{u}) \cdot \nu]=0}
\end{array}\right.
$$

We have

Proposition 2.4. The $2 D$ exterior problem (2.17) where $u \in \mathbb{H}^{n}(\Omega), n \in \mathbb{N}$, has an unique solution $H=-\nabla \varphi$ such that $\varphi \in \mathbf{W}_{2}^{2}\left(\mathbb{R}^{2}\right)$ and $\nabla H \in \mathbb{H}^{n}\left(\mathbb{R}^{2}\right)$. Moreover, we have

$$
\|\varphi\|_{\mathbf{W}_{2}^{2}\left(\mathbb{R}^{2}\right)} \leq C\|u\|_{\mathbb{L}^{2}(\Omega)}, \quad\|\nabla H\|_{\mathbb{H}^{n}\left(\mathbb{R}^{2}\right)} \leq C\|u\|_{\mathbb{H}^{n}(\Omega)} .
$$

Proof. The proof follows the same scheme as Prop. 2.2. Prop 2.1 gives the equivalent norms to gain the continuity and the coercivity in the Lax-Milgram theorem at Step 2.

The second problem writes:

$$
\begin{cases}\operatorname{div} \Delta H=\mathbf{1}_{\Omega} f & \text { in } \mathbb{R}^{2} \\ \operatorname{curl}_{2 D} H=0 & \text { in } \mathbb{R}^{2} \\ {[H \cdot \nu]=0, \quad[(\Delta H) \cdot \nu]=0} & \text { on } \partial \Omega\end{cases}
$$

We have :

Proposition 2.5. The 2D exterior problem (2.18) where $f \in \mathbb{H}^{n}\left(\mathbb{R}^{2}\right), n \in \mathbb{N}$, has an unique solution $H=-\nabla \varphi$ such that $\varphi \in \mathbf{W}_{2}^{2}\left(\mathbb{R}^{2}\right)$ and $\nabla H \in \mathbb{H}^{n}\left(\mathbb{R}^{2}\right)$. Moreover, we have:

$$
\|\varphi\|_{\mathbf{W}_{2}^{2}\left(\mathbb{R}^{2}\right)} \leq C\|f\|_{\mathbb{L}^{2}(\Omega)}, \quad\|\nabla H\|_{\mathbb{H}^{n}\left(\mathbb{R}^{2}\right)} \leq C\|f\|_{\mathbb{H}^{n}\left(\mathbb{R}^{2}\right)} .
$$

Proof. The proof follows the same scheme as the ones of the previous propositions. 
In the case where the magnetic domain is the unitary disk $D(0,1)$ and the magnetic moment $u$ is a constant $2 D$ vector field on $\Omega=D(0,1)$, the magnetostatic equations write:

$$
\begin{cases}\operatorname{div} \mathcal{H}(u)=0 & \text { in } \Omega \cup \Omega^{\prime}, \\ \operatorname{curl} \mathcal{H}(u)=0 & \text { in } \mathbb{R}^{2}, \\ {[\mathcal{H}(u) \cdot \nu]=u \cdot \nu} & \text { on } \partial \Omega,\end{cases}
$$

where $\Omega^{\prime}=\mathbb{R}^{2} \backslash \bar{\Omega}$ and we have:

Proposition 2.6. The equations (2.19) have an unique solution $\mathcal{H}(u)$ in $\mathbb{R}^{2}$, constant on $\Omega=$ $D(0,1)$ and which is given by:

$$
\mathcal{H}(u)(x)=-\frac{u}{2}\left[1-\mathbf{1}_{\Omega^{\prime}}(x)\right]+\frac{1}{2} \mathbf{1}_{\Omega^{\prime}}(x)\left[\frac{x_{1}^{2}-x_{2}^{2}}{|x|^{4}}\left(\begin{array}{c}
u_{1} \\
-u_{2}
\end{array}\right)+\frac{2 x_{1} x_{2}}{|x|^{4}}\left(\begin{array}{l}
u_{2} \\
u_{1}
\end{array}\right)\right],
$$

where $x=\left(x_{1}, x_{2}\right)$ and $u=\left(u_{1}, u_{2}\right)^{t}$.

Proof. Since curl $\mathcal{H}(u)=0$ in $\mathbb{R}^{2}$ there exists a regular function $\varphi$ such that $\mathcal{H}(u)=-\nabla \varphi . \varphi$ then fulfills:

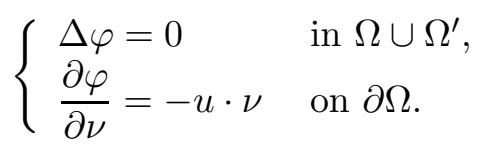

This equation has an unique solution (see [10]):

$$
\varphi(x)=\int_{\partial \Omega} \frac{1}{2 \pi} \ln |x-y|(-u \cdot \nu)(y) d \sigma(y),
$$

and then

$$
\mathcal{H}(u)(x)=\int_{\partial \Omega} \frac{1}{2 \pi}(u \cdot \nu)(y) \frac{x-y}{|x-y|^{2}} d \sigma(y) .
$$

We assume now that the unitary magnetic moment $u$ writes $u=u_{1} e_{1}+u_{2} e_{2}$ and we write the previous equation using the canonical bijection between $\mathbb{R}^{2}$ and $\mathbb{C}$.

Since $\partial \Omega=\left\{y \in \mathbb{R}^{2},|y|=1\right\}=\left\{e^{i \tau}, \tau \in[0,2 \pi[\}\right.$ we have:

$$
\begin{aligned}
\mathcal{H}(u) & =\frac{1}{2 \pi} \int_{0}^{2 \pi}\left(u_{1} \cos \tau+u_{2} \sin \tau\right) \frac{x-e^{i \tau}}{\left|x-\varepsilon^{i \tau}\right|^{2}} d \tau \\
& =\frac{1}{2 \pi} \int_{0}^{2 \pi}\left(u_{1} \frac{e^{i \tau}+e^{-i \tau}}{2}+u_{2} \frac{e^{i \tau}-e^{-i \tau}}{2 i}\right) \frac{x-e^{i \tau}}{\left(x-e^{i \tau}\right)\left(\bar{x}-e^{-i \tau}\right)} d \tau,
\end{aligned}
$$

where $\bar{x}$ is the conjugate complex of $x$. We now let $z=e^{i \tau}$ and remark that $e^{-i \tau}=z^{-1}$ and obtain:

$$
\begin{aligned}
\mathcal{H}(u)(x) & =\frac{1}{4 \pi} \int_{|z|=1}\left[u_{1}\left(z+\frac{1}{z}\right)-i u_{2}\left(z-\frac{1}{z}\right)\right] \frac{1}{\bar{x}-\frac{1}{z}} \frac{d z}{i z} \\
& =\frac{1}{4 i \pi} \int_{|z|=1} \frac{u_{1}\left(z^{2}+1\right)-i u_{2}\left(z^{2}-1\right)}{z(\bar{x} z-1)} d z .
\end{aligned}
$$

Applying the Residue theorem we have

$$
\mathcal{H}(u)=-\frac{1}{2} \operatorname{Ind}_{\partial \Omega}(0)\left[u_{1}+i u_{2}\right]+\frac{1}{2}\left[u_{1}\left(1+\frac{1}{\bar{x}^{2}}\right)-i u_{2}\left(\frac{1}{\bar{x}^{2}}-1\right)\right] \operatorname{Ind}_{\partial \Omega}\left(\frac{1}{\bar{x}}\right),
$$

where $\operatorname{Ind}_{\partial \Omega}(z)=1$ if $z \in \Omega$ and 0 otherwise. Back to the real coordinates we obtain the announced result. 


\subsubsection{Existence of $U^{0}$}

Theorem 2.7. Given $U_{0}^{0} \in \mathbb{H}^{6}(\Omega) \otimes \mathbb{H}_{p}^{7}\left(I_{1}\right)$, such that $\left|U_{0}^{0}\right| \equiv 1$ and $\frac{\partial U_{0}^{0}}{\partial \nu}=0$ on $\partial \Omega \times I_{1}$, there exists $T_{0}>0$ and an unique solution to the equation (2.20):

$$
\left\{\begin{array}{l}
\frac{\partial U^{0}}{\partial t}-\Delta_{\tau} U^{0}=\left|\nabla_{\tau} U^{0}\right|^{2} U^{0}+U^{0} \wedge \Delta_{\tau} U^{0}+U^{0} \wedge H^{0}-U^{0} \wedge\left(U^{0} \wedge H^{0}\right) \text { in } \Omega \times I_{1}, \\
\frac{\partial U^{0}}{\partial \nu}=0 \quad \text { on } \partial \Omega \times I_{1}, \\
U^{0}(t=0)=U_{0}^{0} \quad \text { in } \Omega \times I_{1},
\end{array}\right.
$$

where

$$
\left\{\begin{array}{l}
\operatorname{div}_{\tau}\left(H^{0}+\overline{U^{0}}\right)=0 \\
\operatorname{curl}_{\tau} H^{0}=0
\end{array}\right.
$$

such that $\left|U^{0}\right|=1$ and

$$
U^{0} \in \bigcap_{k=0}^{3}\left[\mathbb{W}^{k, \infty}\left(0, T_{0} ; \mathbb{H}^{6-2 k}(\Omega) \otimes \mathbb{H}_{p}^{7}\left(I_{1}\right)\right) \cap \mathbb{H}^{k}\left(0, T_{0} ; \mathbb{H}^{7-2 k}(\Omega) \otimes \mathbb{H}_{p}^{7}\left(I_{1}\right)\right)\right]
$$

Proof. We apply Prop. 2.2 and obtain that $-H^{0}$ is the projection of $U^{0}$ on the vector field transversal gradients and fulfills:

$$
\left\|H^{0}\right\|_{\mathbb{H}^{s}\left(\mathbb{R}^{2}\right)} \leq C\left\|U^{0}\right\|_{\mathbb{H}^{s}(\Omega)}
$$

In the proof of Prop. 2.2 we only consider the behaviour in the transversal direction, the $z$ coordinate being considered as a parameter. Applying the existence result to the $z$-derivatives of $H^{0}$ we obtain:

$$
\left\|H^{0}\right\|_{\mathbb{H}^{s}(\Omega) \otimes \mathbb{H}_{p}^{t}\left(I_{1}\right)} \leq C\left\|U^{0}\right\|_{\mathbb{H}^{s}(\Omega) \otimes \mathbb{H}_{p}^{t}\left(I_{1}\right)} .
$$

When we consider the $z$-coordinate as a parameter, the proof of existence follows the proof given in [7], we obtain that $U^{0} \in \mathcal{C}\left(\left[0, T_{0}\right] ; \mathbb{H}^{2}\left(\mathbb{R}^{2}\right) \otimes \mathbb{L}_{p}^{2}\left(I_{1}\right)\right) \cap \mathbb{L}^{2}\left(0, T_{0} ; \mathbb{H}^{3}\left(\mathbb{R}^{2}\right) \otimes \mathbb{L}_{p}^{2}\left(I_{1}\right)\right)$. As we need more regularity in time and space (along the transversal direction and the $z$-direction) we use the arguments provided in $[9,21]$ for the existence of the boundary layer terms: we take the derivative of $U^{0}$ with respect to $t$ and apply the previous result. The regularity in time provides regularity on $\Delta_{\tau} U^{0}$ and then enhances the result. We obtain the regularity in the parameter $z$ by taking the derivative in $z$ and applying all the previous steps. We take now the scalar product of the equation with $U^{0}$ and remark that

$$
\Delta_{\tau}\left|U^{0}\right|^{2}=2\left(U^{0} \cdot \Delta_{\tau} U^{0}+\left|\nabla_{\tau} U^{0}\right|^{2}\right)
$$

We obtain:

$$
\partial_{t}\left(\left|U^{0}\right|^{2}-1\right)-\Delta_{\tau}\left(\left|U^{0}\right|^{2}-1\right)=2\left|\nabla_{\tau} U^{0}\right|^{2}\left(\left|U^{0}\right|^{2}-1\right),
$$

which implies $\left|U^{0}\right| \equiv 1$ thanks to Gronwall's lemma as soon as it is fulfilled at $t=0$. 
Theorem 2.8. If $\Omega$ is the unitary disk $D(0,1)$ and the initial data does not depend on $\sigma \in \Omega$, i.e. $U_{0}^{0} \in \mathbb{H}_{p}^{7}\left(I_{1}\right)$ then $U^{0}$ fulfills the following ordinary differential equation with parameter $z \in I_{1}$ :

$$
\left\{\begin{array}{l}
\frac{\partial U^{0}}{\partial t}=U^{0} \wedge H^{0}-U^{0} \wedge\left(U^{0} \wedge H^{0}\right) \quad \text { in } I_{1}, \\
H^{0}=-\frac{1}{2} U_{\tau}^{0} \quad \text { in } I_{1} \\
U^{0}(t=0)=U_{0}^{0} \quad \text { in } I_{1},
\end{array}\right.
$$

which has an unique solution in $\mathcal{C}^{\infty}\left(\mathbb{R}_{t}^{+} ; \mathbb{H}_{p}^{7}\left(I_{1}\right)\right)$.

Proof. Thanks to Prop. 2.6 we have $H^{0}=-\frac{1}{2} U_{\tau}^{0}$ in $\Omega$. We now apply Th. 2.7 and have that $U^{0}$ is regular and $\left|U^{0}\right| \equiv 1$ on $\Omega$. We now prove that the solution does not depend on $\sigma \in \Omega$ (see [7]). We take the scalar product of $(2.20)$ with $-\Delta_{\tau} U^{0}$ in $\mathbb{L}^{2}(\Omega)$, integrate by parts and use (2.21) and the conservation of the norm of $U^{0}$ to obtain:

$$
\frac{1}{2} \frac{d}{d t}\left\|\nabla_{\tau} U^{0}\right\|_{\mathbb{L}^{2}(\Omega)}^{2}+\left\|\Delta_{\tau} U^{0}\right\|_{\mathbb{L}^{2}(\Omega)}^{2} \leq \int_{\Omega}\left|\nabla_{\tau} U^{0}\right|^{4} d x+C\left\|\nabla_{\tau} U^{0}\right\|_{\mathbb{L}^{2}(\Omega)}^{2} .
$$

Since $\left|\nabla_{\tau} U^{0}\right| \in \mathbb{L}^{1}\left(0, T_{0} ; \mathbb{L}^{\infty}(\Omega)\right)$ Gronwall's lemma ensures that $\nabla_{\tau} U^{0} \equiv 0$ since it is fulfilled at $t=0$. So $U^{0}$ does not depend on $\sigma \in \Omega$ and fulfills (2.22). Since $\mathbb{H}_{p}^{7}\left(I_{1}\right)$ is an algebra the Cauchy-Lipschitz theorem gives the local existence and uniqueness of $U^{0} \in \mathcal{C}^{\infty}\left(0, T_{0} ; \mathbb{H}_{p}^{7}\left(I_{1}\right)\right)$. Thanks to successive estimates on the derivates $\nabla_{\tau} U^{0}, \nabla_{\tau}^{2} U^{0}, \ldots$ we prove the global existence of $U^{0}$.

Remark 2.1. The following results state the existence of the next profiles in the asymptotic expansion and of the remainder term for the general case. They remain true in the case of Th. 2.8 for all $T_{0}>0$ since we are limited in the proofs by the existence time of $U^{0}$.

\subsubsection{Existence of $U^{1}$}

Proposition 2.9. Under the assumptions of Th. 2.7 and with the additional assumptions that $U_{0}^{1} \in \mathbb{H}^{5}(\Omega) \otimes \mathbb{H}_{p}^{6}\left(I_{1}\right)$ and $\frac{\partial U_{0}^{1}}{\partial \nu}=0$ on $\partial \Omega \times I_{1}$, there exists an unique solution to

$$
\left\{\begin{aligned}
& \frac{\partial U^{1}}{\partial t}-\Delta_{\tau} U^{1}=\left|\nabla_{\tau} U^{0}\right|^{2} U^{1}+2\left(\nabla_{\tau} U^{0} \cdot \nabla_{\tau} U^{1}\right) U^{0}+U^{0} \wedge \Delta_{\tau} U^{1}+U^{1} \wedge \Delta_{\tau} U^{0} \\
&+U^{0} \wedge H^{1}+U^{1} \wedge H^{0}-U^{0} \wedge\left(U^{0} \wedge H^{1}\right)-U^{0} \wedge\left(U^{1} \wedge H^{0}\right) \\
&-U^{1} \wedge\left(U^{0} \wedge H^{0}\right) \quad \text { in } \Omega \times I_{1}, \\
& \frac{\partial U^{1}}{\partial \nu}=0 \quad \text { on } \partial \Omega \times I_{1}, \\
& U^{1}(0, \cdot)=U_{0}^{1}(\cdot) \quad \text { in } \Omega \times I_{1},
\end{aligned}\right.
$$

where $H^{1}=\mathcal{H}\left(U^{1}\right)+Q^{1}$, solution of Eq. (2.9), fulfills

$$
\left\{\begin{array} { l l } 
{ \operatorname { d i v } _ { \tau } ( \mathcal { H } ( U ^ { 1 } ) + \overline { U ^ { 1 } } ) = 0 } & { \text { in } \mathbb { R } ^ { 2 } \times I _ { 1 } , } \\
{ \operatorname { c u r l } _ { \tau } \mathcal { H } ( U ^ { 1 } ) = 0 } & { \text { in } \mathbb { R } ^ { 2 } \times I _ { 1 } , } \\
{ [ ( \mathcal { H } ( U ^ { 1 } ) + \overline { U ^ { 1 } } ) \cdot \nu ] = 0 } & { \text { on } \partial \Omega \times I _ { 1 } , }
\end{array} \quad \left\{\begin{array}{ll}
\operatorname{div}_{\tau} Q_{\tau}^{1}+\partial_{z} \overline{U_{l}^{0}}=0 & \text { in } \mathbb{R}^{2} \times I_{1}, \\
\operatorname{curl}_{2 D} Q_{\tau}^{1}=0 & \text { in } \mathbb{R}^{2} \times I_{1}, \\
{\left[Q_{\tau}^{1} \cdot \nu\right]=0} & \text { on } \partial \Omega \times I_{1}, \\
\nabla_{\tau} Q_{l}^{1}=\partial_{z} H_{\tau}^{0} & \text { in } \mathbb{R}^{2} \times I_{1}, \\
{\left[Q_{l}^{1}\right]=0} & \text { on } \partial \Omega \times I_{1},
\end{array}\right.\right.
$$


such that

$$
U^{1} \in \bigcap_{k=0}^{2}\left[\mathbb{W}^{k, \infty}\left(0, T_{0} ; \mathbb{H}^{5-2 k}(\Omega) \otimes \mathbb{H}_{p}^{6}\left(I_{1}\right)\right) \cap \mathbb{H}^{k}\left(0, T_{0} ; \mathbb{H}^{6-2 k}(\Omega) \otimes \mathbb{H}_{p}^{6}\left(I_{1}\right)\right)\right] .
$$

Proof. We use the linearity of Eq. (2.9) to split the problem in the two exposed above. We apply Prop. 2.2 to the first exterior problem and we track the dependence on the $z$-coordinate as in the case of $H^{0}=\mathcal{H}\left(U^{0}\right)$ to obtain the existence and regularity of $\mathcal{H}\left(U^{1}\right)$. Thanks to Prop. 2.3 we obtain the existence of $Q_{\tau}^{1}$ and we track the dependence on the parameter $z$. Now we remark that $H_{\tau}^{0}=-\nabla_{\tau} \varphi^{0}$ (according to Prop. 2.2 applied to the solving of $H^{0}$ ). With the dependence on $z$, we have that $\varphi^{0} \in \mathbb{W}^{N, \infty}\left(0, T_{0} ; \mathbb{W}_{1}^{1}\left(\mathbb{R}^{2}\right) \otimes \mathbb{H}_{p}^{6}\left(I_{1}\right)\right)$ and $H^{0}$ has the same regularity as $U^{0}$. As we do not impose an external magnetic field we have that $Q_{l}^{1}=\partial_{z} \varphi^{0}$ and

$$
\begin{aligned}
& Q_{l}^{1} \in \mathbb{W}^{N, \infty}\left(0, T_{0} ; \mathbb{W}_{1}^{1}\left(\mathbb{R}^{2}\right) \otimes \mathbb{H}_{p}^{6}\left(I_{1}\right)\right), \\
& \nabla_{\tau} Q_{l}^{1} \in \bigcap_{k=0}^{3}\left[\mathbb{W}^{k, \infty}\left(0, T_{0} ; \mathbb{H}^{6-2 k}(\Omega) \otimes \mathbb{H}_{p}^{6}\left(I_{1}\right)\right) \cap \mathbb{H}^{k}\left(0, T_{0} ; \mathbb{H}^{7-2 k}(\Omega) \otimes \mathbb{H}_{p}^{6}\left(I_{1}\right)\right)\right],
\end{aligned}
$$

The proof follows then the same scheme as in $[22,23]$ and Th. 2.7.

Remark 2.2. The terms $U^{1}, U^{2}, \ldots$ in the asymptotic expansion take non null values even if their initial data is zero.

\subsubsection{Existence of $U^{2}$}

Proposition 2.10. Under the assumptions of Th. 2.7 and 2.9 and with the extra assumptions $U_{0}^{2} \in \mathbb{H}^{5}(\Omega) \otimes \mathbb{H}_{p}^{5}\left(I_{1}\right)$ and $\frac{\partial U_{0}^{2}}{\partial \nu}=0$ on $\partial \Omega$, there exists an unique solution to

$$
\left\{\begin{aligned}
& \frac{\partial U^{2}}{\partial t}-\Delta_{\tau} U^{2}=\left|\nabla_{\tau} U^{0}\right|^{2} U^{2}+2\left(\nabla_{\tau} U^{0} \cdot \nabla_{\tau} U^{2}\right) U^{0}+U^{0} \wedge \Delta_{\tau} U^{2}+U^{2} \wedge \Delta_{\tau} U^{0} \\
&+U^{0} \wedge H^{2}+U^{2} \wedge H^{0}-U^{0} \wedge\left(U^{0} \wedge H^{2}\right)-U^{0} \wedge\left(U^{2} \wedge H^{0}\right) \\
&-U^{2} \wedge\left(U^{0} \wedge H^{0}\right)+F \quad \text { in } \Omega \times I_{1}, \\
& \frac{\partial U^{2}}{\partial \nu}=0 \quad \text { on } \partial \Omega \times I_{1}, \\
& U^{2}(0, \cdot)=U_{0}^{2}(\cdot) \quad \text { in } \Omega \times I_{1},
\end{aligned}\right.
$$

where

$$
\begin{array}{r}
F=U_{z z}^{0}+\left|U_{z}^{0}\right|^{2} U^{0}+U^{0} \wedge U_{z z}^{0}+U^{1} \wedge \Delta_{\tau} U^{1}+\left|\nabla_{\tau} U^{1}\right|^{2} U^{0}+2\left(\nabla_{\tau} U^{0} \cdot \nabla_{\tau} U^{1}\right) U^{1} \\
+U^{1} \wedge H^{1}-U^{0} \wedge\left(U^{1} \wedge H^{1}\right)-U^{1} \wedge\left(U^{0} \wedge H^{1}\right)-U^{1} \wedge\left(U^{1} \wedge H^{0}\right)
\end{array}
$$

and $H^{2}=\mathcal{H}\left(U^{2}\right)+Q^{2}$, solution of Eq. (2.11), fulfills

$$
\left\{\begin{array} { l l } 
{ \operatorname { d i v } _ { \tau } ( \mathcal { H } ( U ^ { 2 } ) + \overline { U ^ { 2 } } ) = 0 } & { \text { in } \mathbb { R } ^ { 2 } \times I _ { 1 } , } \\
{ \operatorname { c u r l } _ { \tau } \mathcal { H } ( U ^ { 2 } ) = 0 } & { \text { in } \mathbb { R } ^ { 2 } \times I _ { 1 } , } \\
{ [ ( \mathcal { H } ( U ^ { 2 } ) + \overline { U ^ { 2 } } ) \cdot \nu ] = 0 } & { \text { on } \partial \Omega \times I _ { 1 } , }
\end{array} \quad \left\{\begin{array}{ll}
\operatorname{div}_{\tau} Q_{\tau}^{2}+\partial_{z}\left(H_{l}^{1}+\overline{U_{l}^{1}}\right)=0 & \text { in } \mathbb{R}^{2} \times I_{1}, \\
\operatorname{curl}_{2 D} Q_{\tau}^{2}=0 & \text { in } \mathbb{R}^{2} \times I_{1}, \\
{\left[Q_{\tau}^{2} \cdot \nu\right]=0} & \text { on } \partial \Omega \times I_{1}, \\
\nabla_{\tau} Q_{l}^{2}=\partial_{z} H_{\tau}^{1} & \text { in } \Omega \times I_{1}, \\
{\left[Q_{l}^{2}\right]=0} & \text { on } \partial \Omega \times I_{1},
\end{array}\right.\right.
$$

such that

$$
U^{2} \in \bigcap_{k=0}^{2}\left[\mathbb{W}^{k, \infty}\left(0, T_{0} ; \mathbb{H}^{5-2 k}(\Omega) \otimes \mathbb{H}_{p}^{5}\left(I_{1}\right)\right) \cap \mathbb{H}^{k}\left(0, T_{0} ; \mathbb{H}^{6-2 k}(\Omega) \otimes \mathbb{H}_{p}^{5}\left(I_{1}\right)\right)\right]
$$


Proof. We use the linearity to split Eq. (2.11). The existence and regularity of $\mathcal{H}\left(U^{2}\right)$ is given by Prop. 2.2 as in Th 2.9. The solving of $Q_{l}^{2}$ follows the same scheme as in Th. 2.9. We now split the equation on $Q_{\tau}^{2}$ in:

$$
\left\{\begin{array} { l l } 
{ \operatorname { d i v } _ { \tau } Q _ { \tau } ^ { 2 , 1 } + \partial _ { z } \overline { U _ { l } ^ { 1 } } = 0 } & { \text { in } \mathbb { R } ^ { 2 } \times I _ { 1 } , } \\
{ \operatorname { c u r l } _ { 2 D } Q _ { \tau } ^ { 2 , 1 } = 0 } & { \text { in } \mathbb { R } ^ { 2 } \times I _ { 1 } , } \\
{ [ Q _ { \tau } ^ { 2 , 1 } \cdot \nu ] = 0 } & { \text { on } \partial \Omega \times I _ { 1 } }
\end{array} \quad \left\{\begin{array}{ll}
\operatorname{div}_{\tau} Q_{\tau}^{2,2}+\partial_{z} H_{l}^{1}=0 & \text { in } \mathbb{R}^{2} \times I_{1}, \\
\operatorname{curl}_{2 D} Q_{\tau}^{2,2}=0 & \text { in } \mathbb{R}^{2} \times I_{1}, \\
{\left[Q_{\tau}^{2,2} \cdot \nu\right]=0} & \text { on } \partial \Omega \times I_{1},
\end{array}\right.\right.
$$

where $Q^{2}=Q^{2,1}+Q^{2,2}$. Prop. 2.3 and Th. 2.9 give the existence and regularity of $Q^{2,1}$ but we can not apply this proposition to the case of $Q^{2,2}$. As $H_{l}^{1}=Q_{l}^{1}=\partial_{z} \varphi^{0}, Q^{2,2}$ is solution to Eq. (2.17) with $u=\partial_{z}^{2} U^{0}$. Thanks to Prop. 2.4 and with the study of the $z$-regularity, we obtain that $Q_{\tau}^{2,2}=-\nabla_{\tau} \varphi^{2}$ with

$$
\begin{aligned}
& \varphi^{2} \in \mathbb{W}^{3, \infty}\left(0, T_{0} ; \mathbb{W}_{2}^{2}\left(\mathbb{R}^{2}\right) \otimes \mathbb{H}_{p}^{5}\left(I_{1}\right)\right) \\
& \nabla_{\tau} Q^{2,2} \in \bigcap_{k=0}^{3}\left[\mathbb{W}^{k, \infty}\left(0, T_{0} ; \mathbb{H}^{6-2 k}(\Omega) \otimes \mathbb{H}_{p}^{5}\left(I_{1}\right)\right) \cap \mathbb{H}^{k}\left(0, T_{0} ; \mathbb{H}^{7-2 k}(\Omega) \otimes \mathbb{H}_{p}^{5}\left(I_{1}\right)\right)\right] .
\end{aligned}
$$

The proof follows then the same scheme as in $[22,23]$ and Th. 2.7.

\subsubsection{Existence of $U^{3}$}

Proposition 2.11. Under the assumptions of Th. 2.7, 2.9 and 2.10 and with the additional assumptions $U_{0}^{3} \in \mathbb{H}^{5}(\Omega) \otimes \mathbb{H}_{p}^{4}\left(I_{1}\right)$ and $\frac{\partial U_{0}^{3}}{\partial \nu}=0$ on $\partial \Omega$, there exists an unique solution to

$$
\left\{\begin{aligned}
& \frac{\partial U^{3}}{\partial t}-\Delta_{\tau} U^{3}=\left|\nabla_{\tau} U^{0}\right|^{2} U^{3}+2\left(\nabla_{\tau} U^{0} \cdot \nabla_{\tau} U^{3}\right) U^{0}+U^{0} \wedge \Delta_{\tau} U^{3}+U^{3} \wedge \Delta_{\tau} U^{0} \\
&+U^{0} \wedge H^{3}+U^{3} \wedge H^{0}-U^{0} \wedge\left(U^{0} \wedge H^{3}\right)-U^{0} \wedge\left(U^{3} \wedge H^{0}\right) \\
&-U^{3} \wedge\left(U^{0} \wedge H^{0}\right)+F \quad \text { in } \Omega \times I_{1}, \\
& \frac{\partial U^{3}}{\partial \nu}=0 \quad \text { on } \partial \Omega \times I_{1}, \\
& U^{3}(0, \cdot)=U_{0}^{3}(\cdot) \quad \text { in } \Omega \times I_{1},
\end{aligned}\right.
$$

where

$$
\begin{aligned}
& F=\partial_{z}^{2} U^{1}+\left|\nabla_{\tau} U^{1}\right|{ }^{2} U^{0}+2\left(\nabla_{\tau} U^{0} \cdot \nabla_{\tau} U^{1}\right) U^{2}+2\left(\nabla_{\tau} U^{0} \cdot \nabla_{\tau} U^{2}\right) U^{1} \\
&+2\left(\nabla_{\tau} U^{1} \cdot \nabla_{\tau} U^{2}\right) U^{0}+\left|\partial_{z} U^{0}\right|^{2} U^{1}+2\left(\partial_{z} U^{0} \cdot \partial_{z} U^{1}\right) U^{0} \\
&+U^{1} \wedge \partial_{z}^{2} U^{0}+U^{0} \wedge \partial_{z}^{2} U^{1}+U^{1} \wedge \Delta_{\tau} U^{2}+U^{2} \wedge \Delta_{\tau} U^{1} \\
&+U^{1} \wedge H^{2}+U^{2} \wedge H^{1}-U^{0} \wedge\left(U^{1} \wedge H^{2}\right)-U^{0} \wedge\left(U^{2} \wedge H^{1}\right) \\
&-U^{1} \wedge\left(U^{0} \wedge H^{2}\right)-U^{1} \wedge\left(U^{2} \wedge H^{0}\right)-U^{2} \wedge\left(U^{0} \wedge H^{1}\right) \\
&-U^{1} \wedge\left(U^{2} \wedge H^{0}\right)-U^{1} \wedge\left(U^{1} \wedge H^{1}\right)
\end{aligned}
$$

and $H^{3}=\mathcal{H}\left(U^{3}\right)+Q^{3}$, solution of Eq. (2.13), fulfills

$$
\left\{\begin{array} { l l } 
{ \operatorname { d i v } _ { \tau } ( \mathcal { H } ( U ^ { 3 } ) + \overline { U ^ { 3 } } ) = 0 } & { \text { in } \mathbb { R } ^ { 2 } \times I _ { 1 } , } \\
{ \operatorname { c u r l } _ { \tau } \mathcal { H } ( U ^ { 3 } ) = 0 } & { \text { in } \mathbb { R } ^ { 2 } \times I _ { 1 } , } \\
{ [ ( \mathcal { H } ( U ^ { 3 } ) + \overline { U ^ { 3 } } ) \cdot \nu ] = 0 } & { \text { on } \partial \Omega \times I _ { 1 } , }
\end{array} \quad \left\{\begin{array}{ll}
\operatorname{div}_{\tau} Q_{\tau}^{3}+\partial_{z}\left(H_{l}^{2}+\overline{U_{l}^{2}}\right)=0 & \text { in } \mathbb{R}^{2} \times I_{1}, \\
\operatorname{curl}_{2 D} Q_{\tau}^{3}=0 & \text { on } \mathbb{R}^{2} \times I_{1}, \\
\left.Q_{\tau}^{3} \cdot \nu\right]=0 & \text { on } \partial \Omega \times I_{1}, \\
\nabla_{\tau} Q_{l}^{3}=\partial_{z} H_{\tau}^{2} & \text { in } \Omega \times I_{1}, \\
{\left[Q_{l}^{3}\right]=0} & \text { on } \partial \Omega \times I_{1},
\end{array}\right.\right.
$$

such that

$$
U^{3} \in \bigcap_{k=0}^{2}\left[\mathbb{W}^{k, \infty}\left(0, T_{0} ; \mathbb{H}^{5-2 k}(\Omega) \otimes \mathbb{H}_{p}^{4}\left(I_{1}\right)\right) \cap \mathbb{H}^{k}\left(0, T_{0} ; \mathbb{H}^{6-2 k}(\Omega) \otimes \mathbb{H}_{p}^{4}\left(I_{1}\right)\right)\right] .
$$


Proof. We use the linearity to split the equations (2.13). The existence and regularity of $\mathcal{H}\left(U^{3}\right)$ is given by Prop. 2.2 as in Th. 2.9. We follow the same scheme as in Th. 2.9 to obtain $Q_{l}^{3}$, and we split the equation on $Q_{\tau}^{3}$ in:

$$
\left\{\begin{array} { l l } 
{ \operatorname { d i v } _ { \tau } Q _ { \tau } ^ { 3 , 1 } + \partial _ { z } \overline { U _ { l } ^ { 2 } } = 0 } & { \text { in } \mathbb { R } ^ { 2 } \times I _ { 1 } , } \\
{ \operatorname { c u r l } _ { 2 D } Q _ { \tau } ^ { 3 , 1 } = 0 } & { \text { in } \mathbb { R } ^ { 2 } \times I _ { 1 } , } \\
{ [ Q _ { \tau } ^ { 3 , 1 } \cdot \nu ] = 0 } & { \text { on } \partial \Sigma \times I _ { 1 } }
\end{array} \quad \left\{\begin{array}{ll}
\operatorname{div}_{\tau} Q_{\tau}^{3,2}+\partial_{z} H_{l}^{2}=0 & \text { in } \mathbb{R}^{2} \times I_{1}, \\
\operatorname{curl}_{2 D} Q_{\tau}^{3,2}=0 & \text { in } \mathbb{R}^{2} \times I_{1}, \\
{\left[Q_{\tau}^{3,2} \cdot \nu\right]=0} & \text { on } \partial \Sigma \times I_{1},
\end{array}\right.\right.
$$

where $Q^{3}=Q^{3,1}+Q^{3,2}$. Prop. 2.3 and Th. 2.9 give the existence and regularity of $Q^{3,1}$ but wa can not apply this proposition to the case of $Q^{3,2}$. As $H_{l}^{2}=Q_{l}^{2}, Q^{3,2}$ is the sum of the solutions of the equation (2.17) with $u=\partial_{z}^{2} U^{1}$ and of the equation (2.18) with $f=\partial_{z}^{3} \bar{U}_{l}^{0}$. Thanks to Prop. 2.4 and 2.5 and with the study of the $z$-regularity, we obtain that $Q_{\tau}^{3,2}=-\nabla_{\tau} \varphi^{3}$ withavec

$$
\begin{aligned}
& \varphi^{3} \in \mathbb{W}^{3, \infty}\left(0, T_{0} ; \mathbf{W}_{2}^{2}\left(\mathbb{R}^{2}\right) \otimes \mathbb{H}_{p}^{4}\left(I_{1}\right)\right), \\
& \nabla_{\tau} Q^{3,2} \in \bigcap_{k=0}^{3}\left[\mathbb{W}^{k, \infty}\left(0, T_{0} ; \mathbb{H}^{6-2 k}\left(\mathbb{R}^{2}\right) \otimes \mathbb{H}_{p}^{4}\left(I_{1}\right)\right) \cap \mathbb{H}^{k}\left(0, T_{0} ; \mathbb{H}^{7-2 k}\left(\mathbb{R}^{2}\right) \otimes \mathbb{H}_{p}^{4}\left(I_{1}\right)\right)\right] .
\end{aligned}
$$

The proof follows then the same scheme as in $[22,23]$ and Th. 2.7.

\section{Sobolev embeddings and energy estimates in the space $\omega_{\varepsilon}$}

In the following we are lead to perform energy estimates on the remainder terms of $u^{\varepsilon}$ and $H^{\varepsilon}$. These terms will be defined respectively in $\omega_{\varepsilon}$ and $\mathbb{R}^{3}$ (i.e. there is no rescaling) and we need to track the dependence on the domain and particularly on $\varepsilon$ of the Sobolev embeddings and other classical inequalities. The following results are taken from $[22,23,24]$ where they were proved in the case of a thin layer. Thanks to a rescaling (to get rid of the $\varepsilon$ in the definition of the domain), $v(\sigma, z)=u(\varepsilon \sigma, z)$ for $u$ defined on $\omega_{\varepsilon}$, we introduce anisotropic Sobolev spaces on the domain $\omega_{\varepsilon}$. In a first time we remind some anisotropic Sobolev inequalities, the first two being proved in [24], the last one in [22] :

Theorem 3.1 (Anisotropic Agmon's inequality). Let $\left.\Omega_{0}=\right] 0,1\left[{ }^{3}\right.$. There exists an absolute constant $C$ such that

$$
\|u\|_{\mathbb{L}^{\infty}\left(\Omega_{0}\right)} \leq C\|u\|_{\mathbb{L}^{2}\left(\Omega_{0}\right)}^{\frac{1}{4}} \prod_{i=1}^{3}\left(\left\|\frac{\partial^{2} u}{\partial x_{i}^{2}}\right\|_{\mathbb{L}^{2}\left(\Omega_{0}\right)}+\left\|\frac{\partial u}{\partial x_{i}}\right\|_{\mathbb{L}^{2}\left(\Omega_{0}\right)}+\|u\|_{\mathbb{L}^{2}\left(\Omega_{0}\right)}\right)^{\frac{1}{4}},
$$

for all $u \in \mathbb{H}^{2}\left(\Omega_{0}\right)$.

Theorem 3.2 (Anisotropic Ladyzhenskaya's inequality). Let $\left.\Omega_{0}=\prod_{i=1}^{3}\right] 0, \lambda_{i}\left[\right.$ with $\lambda_{i}>0$ for $i=1,2,3$. There exists an absolute constant $C$ such that

$$
\|u\|_{\mathbb{L}^{6}(\Omega)} \leq C \prod_{i=1}^{3}\left(\left\|\frac{\partial u}{\partial x_{i}}\right\|_{\mathbb{L}^{2}(\Omega)}+\frac{1}{\lambda_{i}}\|u\|_{\mathbb{L}^{2}\left(\Omega_{0}\right)}\right)^{\frac{1}{3}}
$$

for all $u \in \mathbb{H}^{1}\left(\Omega_{0}\right)$.

Theorem 3.3. Let $\left.\Omega_{0}=\right] 0,1[3$. There exists an absolute constant $C$ such that

$$
\|u\|_{\mathbb{L}^{12}\left(\Omega_{0}\right)} \leq C \prod_{i=1}^{3}\left(\sum_{j=0}^{1}\left\|\frac{\partial^{j} u}{\partial x_{i}^{j}}\right\|_{\mathbb{L}^{2}\left(\Omega_{0}\right)}\right)^{\frac{1}{4}}\left(\sum_{j=0}^{2}\left\|\frac{\partial^{j} u}{\partial x_{i}^{j}}\right\|_{\mathbb{L}^{2}\left(\Omega_{0}\right)}\right)^{\frac{1}{12}}
$$


We introduce now the anisotropic Sobolev spaces and the anisotropic inequalities.

Definition 3.1. For $1 \leq p<+\infty$, we define the normalized Lebesgues spaces $\mathbb{L}_{\varepsilon}^{p}$ in the following way:

$$
\begin{aligned}
& \mathbb{L}_{\varepsilon}^{p}=\left\{u \in \mathcal{D}^{\prime}\left(\omega_{\varepsilon}\right), \frac{1}{\left|\omega_{\varepsilon}\right|} \int_{\omega_{\varepsilon}}|u(x)|^{p} d x<+\infty\right\}, \\
& |u|_{\varepsilon, p}=\left(\frac{1}{\left|\omega_{\varepsilon}\right|} \int_{\omega_{\varepsilon}}|u(x)|^{p} d x\right)^{1 / p} .
\end{aligned}
$$

We let $\mathbb{W}_{\varepsilon}^{0, p}=\mathbb{L}_{\varepsilon}^{p}$ and we define the anisotropic Sobolev spaces $\mathbb{W}_{\varepsilon}^{m, p}, m \in \mathbb{N}$, and their norms as follows:

$$
\begin{aligned}
& \mathbb{W}_{\varepsilon}^{m+1, p}=\left\{u \in \mathbb{L}_{\varepsilon}^{p}, \partial_{z} u \in \mathbb{W}_{\varepsilon}^{m, p} \text { and }\left(\varepsilon \nabla_{\tau}\right) u \in \mathbb{W}_{\varepsilon}^{m, p}\right\}, \\
& \|u\|_{\mathbb{W}_{\varepsilon}^{m+1, p}}=\left(|u|_{\varepsilon, p}^{p}+\left\|\partial_{z} u\right\|_{\mathbb{W}_{\varepsilon}^{m, p}}^{p}+\left\|\varepsilon \nabla_{\tau} u\right\|_{\mathbb{W}_{\varepsilon}^{m, p}}^{p}\right)^{1 / p} .
\end{aligned}
$$

When $p=2$ we let $\mathbb{H}_{\varepsilon}^{m}=\mathbb{W}_{\varepsilon}^{m, 2}$, and $\|u\|_{\varepsilon, m}=\|u\|_{\mathbb{W}_{\varepsilon}^{m, 2}}$.

When $p=+\infty$, we let:

$$
\begin{aligned}
& \mathbb{L}_{\varepsilon}^{\infty}=\left\{u \in \mathcal{D}^{\prime}\left(\omega_{\varepsilon}\right), \sup _{x \in \omega_{\varepsilon}}|u(x)|<+\infty\right\}, \\
& |u|_{\varepsilon, \infty}=\sup _{x \in \omega_{\varepsilon}}|u(x)| .
\end{aligned}
$$

With $\mathbb{W}_{\varepsilon}^{0, \infty}=\mathbb{L}_{\varepsilon}^{\infty}$, we define the anisotropic Sobolev spaces $\mathbb{W}_{\varepsilon}^{m, \infty}, m \in \mathbb{N}$, and their norms as follows:

$$
\begin{aligned}
& \mathbb{W}_{\varepsilon}^{m+1, \infty}=\left\{u \in \mathbb{L}_{\varepsilon}^{\infty}, \partial_{z} u \in \mathbb{W}_{\varepsilon}^{m, \infty} \text { and }\left(\varepsilon \nabla_{\tau}\right) u \in \mathbb{W}_{\varepsilon}^{m, \infty}\right\}, \\
& \|u\|_{\mathbb{W}_{\varepsilon}^{m+1, \infty}}=\left(|u|_{\varepsilon, \infty}+\left\|\partial_{z} u\right\|_{\mathbb{W}_{\varepsilon}^{m, \infty}}+\left\|\varepsilon \nabla_{\tau} u\right\|_{\mathbb{W}_{\varepsilon}^{m, \infty}}\right) .
\end{aligned}
$$

Corollary 3.4. There exists an $\varepsilon$-independent constant $C$ such that:

- for all $u \in \mathbb{H}_{\varepsilon}^{1}$ and $1 \leq p \leq 6$,

$$
|u|_{\varepsilon, p} \leq C|u|_{\varepsilon, 2}^{\frac{3}{p}-\frac{1}{2}}\|u\|_{\varepsilon, 1}^{\frac{3}{2}-\frac{3}{p}}
$$

- for all $u \in \mathbb{H}_{\varepsilon}^{2}$ and $2 \leq p \leq+\infty$,

$$
|u|_{\varepsilon, p} \leq C|u|_{\varepsilon, 2}^{\frac{1}{4}+\frac{3}{2 p}}\|u\|_{\varepsilon, 2}^{\frac{3}{4}-\frac{3}{2 p}}
$$

- for all $u \in \mathbb{H}_{\varepsilon}^{2}$,

$$
|u|_{\varepsilon, 12} \leq C\|u\|_{\varepsilon, 1}^{\frac{3}{4}}\|u\|_{\varepsilon, 2}^{\frac{1}{4}}
$$

Proof. Thanks to a global map of $\Omega$ we only have to prove the results in $] 0,1\left[{ }^{2} \times\right] 0,1[$.

We obtain the first estimate in the case $p=6$ thanks to theorem 3.2. An interpolation between $\mathbb{L}_{\varepsilon}^{2}$ and $\mathbb{L}_{\varepsilon}^{6}$ give the result.

With the rescaling strategy we use to introduce the anisotropic Sobolev spces we deduce from Th. 3.1 and 3.3 the two inequalities. 


\subsection{Trace and lifting}

Let us remind a definition of the spaces $\mathbb{H}^{s}(\Omega), s=k+\theta>0,0<s<1$ (see $\left.[19,18]\right)$ :

$$
\begin{aligned}
& \mathbb{H}^{s}(\Omega)=\left\{u \in \mathbb{H}^{k}(\Omega), \int_{\Omega} \int_{\Omega} \frac{\left|D^{\alpha} u(x)-D^{\alpha} u(y)\right|^{2}}{|x-y|^{n+2 s}} d x d y<+\infty, \forall|\alpha|=k\right\}, \\
& \|u\|_{\mathbb{H}^{s}(\Omega)}=\left(\|u\|_{\mathbb{H}^{k}(\Omega)}^{2}+\sum_{|\alpha|=k} \int_{\Omega} \int_{\Omega} \frac{\left|D^{\alpha} u(x)-D^{\alpha} u(y)\right|^{2}}{|x-y|^{n+2 s}} d x d y\right)^{1 / 2} .
\end{aligned}
$$

With the rescaling strategy we use for the $\mathbb{H}^{p}$ spaces we define anisotropic Sobolev spaces on the boundary $\partial \omega_{\varepsilon}=\varepsilon \Omega \times I_{1}$ (where the periodic domain $I_{1}$ has no boundary):

$$
\begin{aligned}
& \mathbb{H}_{\varepsilon}^{s}\left(\partial \omega_{\varepsilon}\right)=\left\{u \in \mathbb{H}_{\varepsilon}^{k}\left(\partial \omega_{\varepsilon}\right), \frac{1}{\left|\partial \omega_{\varepsilon}\right|^{2}} \int_{\partial \omega_{\varepsilon}} \int_{\partial \omega_{\varepsilon}} \frac{\left|D_{\varepsilon}^{\alpha} u(y)-D_{\varepsilon}^{\alpha} u\left(y^{\prime}\right)\right|^{2}}{\left(\varepsilon^{-2}\left|\sigma-\sigma^{\prime}\right|^{2}+\left|z-z^{\prime}\right|^{2}\right)^{n / 2+s}} d y d y^{\prime}<+\infty, \forall|\alpha|=k\right\}, \\
& \|u\|_{\mathbb{H}_{\varepsilon}^{s}\left(\partial \omega_{\varepsilon}\right)}=\left(\|u\|_{\mathbb{H}^{k}\left(\partial \omega_{\varepsilon}\right)}^{2}+\sum_{|\alpha|=k} \frac{1}{\left|\partial \omega_{\varepsilon}\right|^{2}} \int_{\partial \omega_{\varepsilon}} \int_{\partial \omega_{\varepsilon}} \frac{\left|D_{\varepsilon}^{\alpha} u(y)-D_{\varepsilon}^{\alpha} u\left(y^{\prime}\right)\right|^{2}}{\left(\varepsilon^{-2}\left|\sigma-\sigma^{\prime}\right|^{2}+\left|z-z^{\prime}\right|^{2}\right)^{n / 2+s}} d y d y^{\prime}\right)^{1 / 2},
\end{aligned}
$$

where $y=(\sigma, z), y^{\prime}=\left(\sigma^{\prime}, z^{\prime}\right), D_{\varepsilon}=\left(\varepsilon \nabla_{\tau}^{t}, \partial_{z}\right)^{t}$ and $\alpha \in \mathbb{N}^{k}$.

Lemma 3.5. There exists an $\varepsilon$-independent constant $C$ such that for all $\varepsilon>0$ small enough and $u \in \mathbb{H}_{\varepsilon}^{1}$,

$$
\|u\|_{\mathbb{H}_{\varepsilon}^{\frac{1}{2}}\left(\partial \omega_{\varepsilon}\right)} \leq C\|u\|_{\varepsilon, 1} .
$$

There exists an $\varepsilon$-independant constant $C$ such that for all $0<\varepsilon$ small enough and $f \in \mathbb{H}_{\varepsilon}^{\frac{1}{2}}\left(\partial \omega_{\varepsilon}\right)$, there exists a lifting $\psi \in \mathbb{H}_{\varepsilon}^{1}$ of $f$ such that:

$$
\|\psi\|_{\varepsilon, 1} \leq C\|f\|_{\mathbb{H}_{\varepsilon}^{\frac{1}{2}}\left(\partial \omega_{\varepsilon}\right)} .
$$

Proof. Anisotropic trace theorem: for all $(\sigma, x) \in \Omega \times I_{1}$ we let $v(\sigma, x)=u(\varepsilon \sigma, z)$. We now apply the classical trace theorem to $v$ and we get:

$$
\|v\|_{\mathbb{H}^{\frac{1}{2}\left(\partial \Omega \times I_{1}\right)}} \leq c\|v\|_{\mathbb{H}^{1}\left(\Omega \times I_{1}\right)},
$$

where $c$ does not depend on $\varepsilon$. We now perform some estimates on $v$ : as in Lemma 3.4 we obtain that there exists some $\varepsilon$-independant constants $C$ and $C^{\prime}$ such that:

$$
C\|u\|_{\varepsilon, 1} \leq\|v\|_{\mathbb{H}^{1}\left(\Omega \times I_{1}\right)} \leq C^{\prime}\|u\|_{\varepsilon, 1} .
$$

As the spaces $\mathbb{H}_{\varepsilon}^{\frac{1}{2}}\left(\partial \omega_{\varepsilon}\right)$ are defined such that they take into account the anisotropy of the domain, we obtain th following estimates on the norm $\mathbb{H}^{\frac{1}{2}}$ :

$$
C\|u\|_{\mathbb{H}_{\varepsilon}^{\frac{1}{2}}\left(\partial \omega_{\varepsilon}\right)} \leq\|v\|_{\mathbb{H}^{\frac{1}{2}}\left(\partial \Omega \times I_{1}\right)} \leq C^{\prime}\|u\|_{\mathbb{H}_{\varepsilon}^{\frac{1}{2}}\left(\partial \omega_{\varepsilon}\right)},
$$

which gives the result.

Anisotropic lifting theorem : In the same way we apply the classical lifting theorem to $g(\sigma, z)=f(\varepsilon \sigma, z),(\sigma, x) \in \partial \Omega \times I_{1}$ and we obtain a lifting $v \in \mathbb{H}^{1}\left(\Omega \times I_{1}\right)$. We define then $u$ by $u(\varepsilon \sigma, z)=v(\sigma, z)$ and $u$ is a lifting of $f \in \mathbb{H}^{\frac{1}{2}}\left(\partial \omega_{\varepsilon}\right)$, the previous estimates conclude the proof. 


\subsection{Some inequalities}

In the following we need the following results :

Lemma 3.6. Let $\Omega$ a regular open bounded set of $\mathbb{R}^{n}$. There exists a constant $C$ such that for all $u \in \mathbb{H}^{2}(\Omega)$ such that $\frac{\partial u}{\partial \nu}=0$ on $\partial \Omega$, we have:

$$
\|u\|_{\mathbb{H}^{2}(\Omega)} \leq C\left(\|u\|_{\mathbb{L}^{2}(\Omega)}^{2}+\|\Delta u\|_{\mathbb{L}^{2}(\Omega)}^{2}\right)^{1 / 2} .
$$

There exist a constant $C$ such that for all $u \in \mathbb{H}^{m}(\Omega), m \in \mathbb{N}^{*}$, we have:

$$
\|u\|_{\mathbb{H}^{m}(\Omega)} \leq C\left(\|u\|_{\mathbb{L}^{2}(\Omega)}+\|\operatorname{div} u\|_{\mathbb{H}^{m-1}(\Omega)}+\|\operatorname{curl} u\|_{\mathbb{H}^{m-1}(\Omega)}+\|u \cdot \nu\|_{\mathbb{H}^{m-1 / 2}(\partial \Omega)}\right) .
$$

Proof. The first inequality comes from the regularity of the operator $I-\Delta$ with domain

$$
D(I-\Delta)=\left\{u \in \mathbb{H}^{2}(\Omega), \frac{\partial u}{\partial \nu}=0 \text { sur } \partial \Omega\right\}
$$

(cf $[10])$. The second inequality is proved in [11].

Remark 3.1. The second result remains true with $u \wedge \nu$ instead of $u \cdot \nu$ and in a domain with bounded complementary instead of a bounded set.

We also use anisotropic versions of these results:

Lemma 3.7. For all $u \in \mathbb{H}_{\varepsilon}^{2}$ such that $\frac{\partial u}{\partial n}=0$ on $\partial \omega_{\varepsilon}$, there exists an $\varepsilon$-independent constant C such that

$$
\|u\|_{\varepsilon, 2} \leq C\left(|u|_{\varepsilon, 2}^{2}+\left|\partial_{z}^{2} u\right|_{\varepsilon, 2}^{2}+\left|\varepsilon^{2} \Delta_{\tau} u\right|_{\varepsilon, 2}^{2}\right)^{1 / 2}
$$

For all $u \in \mathbb{H}_{\varepsilon}^{m}, m \in \mathbb{N}^{*}$, there exists an $\varepsilon$-independent constant $C$ such that:

$$
\begin{aligned}
\|u\|_{\varepsilon, m} \leq & C\left(|u|_{\varepsilon, 2}+\left\|\partial_{z} u_{l}\right\|_{\varepsilon, m-1}+\left\|\varepsilon \operatorname{div}_{\tau} u\right\|_{\varepsilon, m-1}+\left\|\left(e_{3} \partial_{z}\right) \wedge u+\varepsilon \operatorname{curl}_{\tau} u\right\|_{\varepsilon, m-1}\right. \\
& \left.+\|u \cdot \nu\|_{\mathbb{H}_{\varepsilon}^{m-\frac{1}{2}}\left(\partial \omega_{\varepsilon}\right)}\right) .
\end{aligned}
$$

Proof. We let $v(\sigma, z)=u(\varepsilon \sigma, z)$ to consider the domain $\Omega \times I_{1}$. We then apply Lemma 3.6 to $v$ and we deduce the result thanks to Lemma 3.5.

Corollary 3.8. There exists an $\varepsilon$-independent constant $C$ such that for all $u \in \mathbb{H}_{\varepsilon}^{2}$ satisfying $\frac{\partial u}{\partial \nu}=0$ on $\partial \omega_{\varepsilon}$ we have:

$$
\left\|\left(e_{3} \partial_{z}+\varepsilon \nabla_{\tau}\right) u\right\|_{\varepsilon, 1} \leq C\left(\left|\partial_{z} u\right|_{\varepsilon, 2}+\left|\varepsilon \nabla_{\tau} u\right|_{\varepsilon, 2}+\left|\partial_{z}^{2} u\right|_{\varepsilon, 2}+\left|\varepsilon^{2} \Delta_{\tau} u\right|_{\varepsilon, 2}\right) .
$$

Moreover if $u \in \mathbb{H}_{\varepsilon}^{3}$, there exists an $\varepsilon$-independent constant $C$ such that:

$$
\left\|\left(e_{3} \partial_{z}+\varepsilon \nabla_{\tau}\right) u\right\|_{\varepsilon, 2} \leq C\left(\left|\partial_{z} u\right|_{\varepsilon, 2}+\left|\varepsilon \nabla_{\tau} u\right|_{\varepsilon, 2}+\left\|\partial_{z}^{2} u\right\|_{\varepsilon, 1}+\left\|\varepsilon^{2} \Delta_{\tau} u\right\|_{\varepsilon, 1}\right) .
$$


We let:

- in $\omega_{\varepsilon}$,

$$
\left\{\begin{array}{l}
u^{\varepsilon}(t, x)=U^{0}\left(t, \frac{\sigma}{\varepsilon}, z\right)+\varepsilon U^{1}\left(t, \frac{\sigma}{\varepsilon}, z\right)+\varepsilon^{2} U^{2}\left(t, \frac{\sigma}{\varepsilon}, z\right)+\varepsilon^{3} U^{3}\left(t, \frac{\sigma}{\varepsilon}, z\right)+\varepsilon^{3} r^{\varepsilon}(t, x), \\
H^{\varepsilon}(t, x)=H^{0}\left(t, \frac{\sigma}{\varepsilon}, z\right)+\varepsilon H^{1}\left(t, \frac{\sigma}{\varepsilon}, z\right)+\varepsilon^{2} H^{2}\left(t, \frac{\sigma}{\varepsilon}, z\right)+\varepsilon^{3} H^{3}\left(t, \frac{\sigma}{\varepsilon}, z\right)+\varepsilon^{3} Q^{\varepsilon}(t, x),
\end{array}\right.
$$

- in $\left(\mathbb{R}^{2} \times I_{1}\right) \backslash \omega_{\varepsilon}$

$$
H^{\varepsilon}(t, x)=H^{0}\left(t, \frac{\sigma}{\varepsilon}, z\right)+\varepsilon H^{1}\left(t, \frac{\sigma}{\varepsilon}, z\right)+\varepsilon^{2} H^{2}\left(t, \frac{\sigma}{\varepsilon}, z\right)+\varepsilon^{3} H^{3}\left(t, \frac{\sigma}{\varepsilon}, z\right)+\varepsilon^{3} Q^{\varepsilon}(t, x),
$$

where $r^{\varepsilon}$ and $Q^{\varepsilon}$ are the remainder terms of $u$ and $H$ and $x=(\sigma, z)$. In the following we let $\widetilde{V}(x)=V\left(\frac{\sigma}{\varepsilon}, z\right)$.

\subsection{Remainder term of $H^{\varepsilon}$}

Back in Eqs. (1.2) and (2.5) we simplify by using the equations fulfilled by the profiles and we obtain:

$$
\begin{cases}\operatorname{div}\left(Q^{\varepsilon}+\overline{r^{\varepsilon}}\right)+\partial_{z}\left(\widetilde{H_{l}^{3}}+\widetilde{\overline{U_{l}^{3}}}\right)=0 & \text { in } \mathbb{R}^{2} \times I_{1} \\ \operatorname{curl} Q^{\varepsilon}+\partial_{z}\left(e_{3} \wedge \widetilde{H^{3}}\right)=0 & \text { in } \mathbb{R}^{2} \times I_{1} \\ {\left[\left(Q^{\varepsilon}+\overline{r^{\varepsilon}}\right) \cdot \nu\right]=0} & \text { on } \partial \omega_{\varepsilon} \\ {\left[Q^{\varepsilon} \wedge \nu\right]=0} & \text { on } \partial \omega_{\varepsilon}\end{cases}
$$

We then consider the remainder $Q^{\varepsilon}$ as a function of $r^{\varepsilon}$ to get:

Theorem 4.1. Under the assumptions of Th. 2.7, 2.9, 2.10 and 2.11 and if $r^{\varepsilon} \in \mathbb{H}_{\varepsilon}^{p}, p \in \mathbb{N}$, there exists an unique $Q^{\varepsilon}=R^{\varepsilon}+S^{\varepsilon}$ such that

$$
\left\{\begin{array} { l l } 
{ \operatorname { d i v } ( R ^ { \varepsilon } + \overline { r ^ { \varepsilon } } ) = 0 } & { \text { in } \mathbb { R } ^ { 2 } \times I _ { 1 } , } \\
{ \operatorname { c u r l } R ^ { \varepsilon } = 0 } & { \text { in } \mathbb { R } ^ { 2 } \times I _ { 1 } , } \\
{ [ ( R ^ { \varepsilon } + \overline { r ^ { \varepsilon } } ) \cdot \nu ] = 0 } & { \text { on } \partial \omega _ { \varepsilon } , } \\
{ [ R ^ { \varepsilon } \wedge \nu ] = 0 } & { \text { on } \partial \omega _ { \varepsilon } , }
\end{array} \quad \left\{\begin{array}{ll}
\operatorname{div} S^{\varepsilon}+\partial_{z}\left(\widetilde{H_{l}^{3}}+\widetilde{\widetilde{U_{l}^{3}}}\right) & \text { in } \mathbb{R}^{2} \times I_{1}, \\
\operatorname{curl} S^{\varepsilon}+\partial_{z}\left(e_{3} \wedge \widetilde{H^{3}}\right) & \text { in } \mathbb{R}^{3}, \\
{\left[S^{\varepsilon} \cdot \nu\right]=0} & \text { on } \partial \omega_{\varepsilon}, \\
{\left[S^{\varepsilon} \wedge \nu\right]=0} & \text { on } \partial \omega_{\varepsilon},
\end{array}\right.\right.
$$

and there exists some $\varepsilon$ independent constant $c_{0}$ such that

$$
\begin{aligned}
& \left\|R^{\varepsilon}\right\|_{\varepsilon, p} \leq c_{0}\left\|r^{\varepsilon}\right\|_{\varepsilon, p}, \\
& \left\|S^{\varepsilon}\right\|_{\varepsilon, 3} \leq c_{0} .
\end{aligned}
$$

Proof. See Appendix A 


\subsection{Remainder term of $u^{\varepsilon}$}

According to Th. 4.1 we let:

$$
\left\{\begin{array}{l}
a^{\varepsilon}(t, x)=U^{0}\left(t, \frac{\sigma}{\varepsilon}, z\right)+\varepsilon U^{1}\left(t, \frac{\sigma}{\varepsilon}, z\right)+\varepsilon^{2} U^{2}\left(t, \frac{\sigma}{\varepsilon}, z\right)+\varepsilon^{3} U^{3}\left(t, \frac{\sigma}{\varepsilon}, z\right) \quad \text { in } \omega_{\varepsilon}, \\
b^{\varepsilon}(t, x)=U^{1}\left(t, \frac{\sigma}{\varepsilon}, z\right)+\varepsilon U^{2}\left(t, \frac{\sigma}{\varepsilon}, z\right)+\varepsilon^{2} U^{3}\left(t, \frac{\sigma}{\varepsilon}, z\right) \quad \text { in } \omega_{\varepsilon}, \\
c^{\varepsilon}(t, x)=U^{2}\left(t, \frac{\sigma}{\varepsilon}, z\right)+\varepsilon U^{3}\left(t, \frac{\sigma}{\varepsilon}, z\right) \quad \text { in } \omega_{\varepsilon}, \\
Q^{\varepsilon}=R^{\varepsilon}+S^{\varepsilon} \quad \text { in } \omega_{\varepsilon}, \\
K^{\varepsilon}(t, x)=H^{0}\left(t, \frac{\sigma}{\varepsilon}, z\right)+\varepsilon H^{1}\left(t, \frac{\sigma}{\varepsilon}, z\right)+\varepsilon^{2} H^{2}\left(t, \frac{\sigma}{\varepsilon}, z\right)+\varepsilon^{3} U^{3}\left(t, \frac{\sigma}{\varepsilon}, z\right)+\varepsilon^{3} S^{\varepsilon} \quad \text { in } \omega_{\varepsilon},
\end{array}\right.
$$

Back in Eq. (2.4) we simplify by using the equations of the profiles (2.8), (2.10), (2.12) and (2.14). We obtain :

$$
\left\{\begin{array}{l}
\frac{\partial r^{\varepsilon}}{\partial t}-\varepsilon^{2} \Delta r^{\varepsilon}=T_{1}+\cdots+T_{8}+F^{\varepsilon} \quad \text { in } \omega_{\varepsilon} \\
\frac{\partial r^{\varepsilon}}{\partial \nu}=0 \quad \text { on } \partial \omega_{\varepsilon}, \\
r^{\varepsilon}(0, x)=r_{0}^{\varepsilon}(x) \quad \text { in } \omega_{\varepsilon},
\end{array}\right.
$$

where

$$
\left\{\begin{array}{l}
T_{1}=\varepsilon^{8}\left|\nabla r^{\varepsilon}\right|^{2} r^{\varepsilon}, \\
T_{2}=\varepsilon^{5}\left(\left|\nabla r^{\varepsilon}\right|^{2} a^{\varepsilon}+2\left(\nabla r^{\varepsilon} \cdot \nabla a^{\varepsilon}\right) r^{\varepsilon}\right), \\
T_{3}=\varepsilon^{2}\left(\left|\nabla a^{\varepsilon}\right|^{2} r^{\varepsilon}+2\left(\nabla r^{\varepsilon} \cdot \nabla a^{\varepsilon}\right) a^{\varepsilon}\right), \\
T_{4}=\varepsilon^{2}\left(r^{\varepsilon} \wedge \Delta a^{\varepsilon}+a^{\varepsilon} \wedge \Delta r^{\varepsilon}+\varepsilon^{3} r^{\varepsilon} \wedge \Delta r^{\varepsilon}\right), \\
T_{5}=a^{\varepsilon} \wedge R^{\varepsilon}+r^{\varepsilon} \wedge K^{\varepsilon}+\varepsilon^{3} r^{\varepsilon} \wedge R^{\varepsilon}, \\
T_{6}=-\left[a^{\varepsilon} \wedge\left(a^{\varepsilon} \wedge R^{\varepsilon}\right)+a^{\varepsilon} \wedge\left(r^{\varepsilon} \wedge K^{\varepsilon}\right)+r^{\varepsilon} \wedge\left(a^{\varepsilon} \wedge K^{\varepsilon}\right)\right], \\
T_{7}=-\varepsilon^{3}\left[r^{\varepsilon} \wedge\left(r^{\varepsilon} \wedge K^{\varepsilon}\right)+r^{\varepsilon} \wedge\left(a^{\varepsilon} \wedge R^{\varepsilon}\right)+a^{\varepsilon} \wedge\left(r^{\varepsilon} \wedge R^{\varepsilon}\right)\right], \\
T_{8}=-\varepsilon^{6} r^{\varepsilon} \wedge\left(r^{\varepsilon} \wedge R^{\varepsilon}\right),
\end{array}\right.
$$

and $F^{\varepsilon}=\varepsilon A+a^{\varepsilon} \wedge S^{\varepsilon}-a^{\varepsilon} \wedge\left(a^{\varepsilon} \wedge S^{\varepsilon}\right)$ where $A$ is the sum of products of the profiles and their derivatives, both valued in $\left(\frac{x}{\varepsilon}, z\right)$ (See Appendix B).

According to the regularity results we obtained on the profiles, we have the following proposition:

Proposition 4.2. Under the assumptions of Th. 2.7, 2.9, 2.10 and 2.11, for all $p, 1 \leq p \leq+\infty$ and for all $0<T<T_{0}$, there exists some $\varepsilon$-independent and positive constants $C_{p}$ such that for all $\varepsilon>0, t \in[0, T]$, and $i=0,1,2$,

$$
\left\|a^{\varepsilon}(t, .)\right\|_{\mathbb{W}_{\varepsilon}^{i, p}} \leq C_{p}
$$


For all $p, 2 \leq p<+\infty$ and for all $0<T<T_{0}$ there exists some $\varepsilon$-independent positive constants $C_{p}$ such that for all $\varepsilon>0$ and $t \in[0, T]$,

$$
\left\|a^{\varepsilon}(t, .)\right\|_{\mathbb{W}_{\varepsilon}^{3, p}} \leq C_{p}
$$

For all $p, 1 \leq p \leq+\infty$ and $0<T<T_{0}$ there exists some $\varepsilon$-independent positive constants $C_{p}$ such that for all $\varepsilon>0$ and $t \in[0, T]$,

$$
\begin{aligned}
& \left\|K^{\varepsilon}(t, .)\right\|_{\mathbb{W}_{\varepsilon}^{1, p}} \leq C_{p}, \\
& \left\|F^{\varepsilon}(t, .)\right\|_{\varepsilon, 1} \leq C_{2} .
\end{aligned}
$$

Proof. Thanks to the regularity results we have some estimates on the profiles in the spaces $\mathbb{W}^{k, \infty}\left(\Omega \times I_{1}\right)$. Thanks to a rescaling we are lead to consider the anisotropic spaces $\mathbb{W}_{\varepsilon}^{k, \infty}$. An interpolation with the estimates obtained in $\mathbb{L}_{\varepsilon}^{2}$ and the estimates performed on $S^{\varepsilon}$ in Th. 4.1 give the result.

\subsection{Estimate on the remainder term}

We will now perform some estimates on the remainder term $r^{\varepsilon}$. This will provides us a proof of the existence of $u^{\varepsilon}$ for a more regular initial data $\left(\mathbb{H}^{6}(\Omega) \otimes \mathbb{H}^{7}\left(I_{1}\right)\right)$ in the case of straight wires (instead of $\mathbb{H}^{2}$ in [7]) and also an asymptotic expansion of $u^{\varepsilon}$. The loss of regularity of the initial data is typical of the asymptotic expansion method we use (see also [5, 9, 12, 21, 22, 23]). These estimates are performed on a Galerkin approximation which will allow us to justify all integration by parts. The approximation space we consider for the Landau-Lifschitz equation is built on the basis of eigenfunctions of the Laplace operator solved on the domain $\omega_{\varepsilon}=\varepsilon \Omega \times I_{1}$ and with domain $\left\{u \in \mathbb{H}_{\varepsilon}^{2}, \frac{\partial u}{\partial n}=0\right.$ on $\left.\partial \omega_{\varepsilon}\right\}$. We also use this basis for the magnetic field thanks to the projection operator (we will not detail this step). In the following we then have to prove estimates on $r_{n}^{\varepsilon}$, the projection of $r^{\varepsilon}$ on the approximation space, that are independent of $\varepsilon$. We let:

$$
\begin{aligned}
\mathbf{Q}(t)= & \left|r_{n}^{\varepsilon}(t, .)\right|_{\varepsilon, 2}^{2}+\left|\partial_{z} r_{n}^{\varepsilon}(t, .)\right|_{\varepsilon, 2}^{2}+\left|\varepsilon \nabla_{\tau} r_{n}^{\varepsilon}(t, .)\right|_{\varepsilon, 2}^{2} \\
& +\varepsilon^{2}\left(\left|\partial_{z}^{2} r_{n}^{\varepsilon}(t, .)\right|_{\varepsilon, 2}^{2}+\left|\varepsilon \nabla_{\tau} \partial_{z} r_{n}^{\varepsilon}\right|_{\varepsilon, 2}^{2}+\left|\varepsilon^{2} \Delta_{\tau} r_{n}^{\varepsilon}(t, .)\right|_{\varepsilon, 2}^{2}\right),
\end{aligned}
$$

and we assume that $\mathbf{Q}(0) \leq\left\|r_{0}^{\varepsilon}\right\|_{\varepsilon, 1}^{2}+\varepsilon^{2}\left\|r^{\varepsilon}\right\|_{\varepsilon, 2}^{2}$ is bounded.

As we consider a Galerkin approximation of $r^{\varepsilon}$ we get the existence of $\mathbf{Q}$ on a maximum time interval $\left[0, T_{n, \varepsilon}^{*}\left[\right.\right.$ where $0<T_{n, \varepsilon}^{*}<T_{0}$ and $T_{0}$ is the existence time of $U^{0}$. We want to prove here the existence of $\varepsilon_{T}$ small enough such that we have the existence of $\mathbf{Q}$ on $[0, T]$ independently of $n$ if $0<\varepsilon \leq \varepsilon_{T}$. We also show the estimates of the main result. We will proceed as it follows: We let $0<T<T_{0}$ and we perform energy estimates independtly of $n$, we take the scalar product in $\mathbb{L}_{\varepsilon}^{2}$ of Eq. (4.2) with $r^{\varepsilon}, \partial_{z}^{2} r^{\varepsilon}+\varepsilon^{2} \Delta_{\tau} r^{\varepsilon}$ and $\varepsilon^{2}\left(\partial_{z}^{4}+\varepsilon^{2} \partial_{z}^{2} \Delta_{\tau}+\varepsilon^{4} \Delta_{\tau}^{2}\right) r^{\varepsilon}$. By integrating by parts and absorbing the cumbersome terms thanks to the dissipative term $-\varepsilon^{2} \Delta r^{\varepsilon}$ in Eq. (4.2) we prove (see Appendix $\mathrm{C}$ ) that $\mathbf{Q}$ fulfills on $[0, T] \cap\left[0, T_{n, \varepsilon}^{*}[\right.$ the differential inequality:

$$
\mathbf{Q}^{\prime}(t) \leq C_{T}(1+\mathbf{Q}(t))+\varepsilon^{2} P_{T}(\mathbf{Q}(t)), \quad t \in[0, T] \cap\left[0, T_{n, \varepsilon}^{*}[,\right.
$$

where $C_{T}$ is a constant and $P_{T}$ a polynomial, both independent from $n$ and $\varepsilon$ (but not from $T$ ). We let now

$$
T^{\varepsilon}=\sup \left\{T, T<T_{0}, P(\mathbf{Q}(t)) \leq \frac{1}{\varepsilon^{2}}, \forall t \in[0, T]\right\} .
$$


For all $t<T<T^{\varepsilon}, \mathbf{Q}^{\prime} \leq C(1+\mathbf{Q})$, and

$$
\mathbf{Q}(t) \leq(1+\mathbf{Q}(0)) \exp (C t)-1
$$

thanks to the Gronwall lemma. We then deduce that $P(\mathbf{Q}(t)) \leq \frac{1}{\varepsilon^{2}}$ for all $t \leq \min \left(T_{0}, a \ln \frac{1}{\varepsilon}+b\right)$. We then have that $\lim _{\varepsilon \rightarrow 0} T_{\varepsilon}=T_{0}$, which implies $T_{n, \varepsilon}^{*} \geq T$ as soon as $\left.\left.\varepsilon \in\right] 0, \varepsilon_{T}\right]$ where $\varepsilon_{T}$ is a constant small enough. Moreover $r^{\varepsilon}$ is bounded independtly from $\varepsilon$ in $\mathbb{L}^{\infty}\left(0, T ; \mathbb{H}_{\varepsilon}^{1}\right)$ and $\varepsilon r^{\varepsilon}$ is bounded independently from $\varepsilon$ in $\mathbb{L}^{\infty}\left(0, T ; \mathbb{H}_{\varepsilon}^{2}\right)$ for all $0<T<T_{\varepsilon}$.

The anisotropic Sobolev embeddings and the renormalization in the Lebesgue spaces give the main result.

\section{Remark 4.1.}

In the case of Th. 2.8 where the domain is the unitary disk and the initial data does not depend on $\sigma \in \Omega$, this proof remains valid and moreover give that the existence time $T^{\varepsilon}$ of $r^{\varepsilon}$ goes to infinity as $\varepsilon$ goes to zero.

Acknowledgments: The author wishes to thanks his Ph.D. advisors G. Carbou and P. Fabrie for their help. He is also grateful to A. Thiaville and J. Miltat for fruitful discussions and for the suggestion of this topic.

\section{A Proof of Theorem 4.1}

Under the assumptions of Th. 2.7, 2.9, 2.10 and 2.11 and if $r^{\varepsilon} \in \mathbb{H}_{\varepsilon}^{p}, p \in \mathbb{N}$, there exists an unique $Q^{\varepsilon}=R^{\varepsilon}+S^{\varepsilon}$ such that

$$
\left\{\begin{array} { l l } 
{ \operatorname { d i v } ( R ^ { \varepsilon } + \overline { r ^ { \varepsilon } } ) = 0 } & { \text { in } \mathbb { R } ^ { 2 } \times I _ { 1 } , } \\
{ \operatorname { c u r l } R ^ { \varepsilon } = 0 } & { \text { in } \mathbb { R } ^ { 2 } \times I _ { 1 } , } \\
{ [ ( R ^ { \varepsilon } + \overline { r ^ { \varepsilon } } ) \cdot \nu ] = 0 } & { \text { on } \partial \omega _ { \varepsilon } , } \\
{ [ R ^ { \varepsilon } \wedge \nu ] = 0 } & { \text { on } \partial \omega _ { \varepsilon } , }
\end{array} \quad \left\{\begin{array}{ll}
\operatorname{div} S^{\varepsilon}+\partial_{z}\left(\widetilde{H_{l}^{3}}+\widetilde{\widetilde{U_{l}^{3}}}\right) & \text { in } \mathbb{R}^{2} \times I_{1}, \\
\operatorname{curl} S^{\varepsilon}+\partial_{z}\left(e_{3} \wedge \widetilde{H^{3}}\right) & \text { in } \mathbb{R}^{3}, \\
{\left[S^{\varepsilon} \cdot \nu\right]=0} & \text { on } \partial \omega_{\varepsilon}, \\
{\left[S^{\varepsilon} \wedge \nu\right]=0} & \text { on } \partial \omega_{\varepsilon},
\end{array}\right.\right.
$$

and there exists some $\varepsilon$ independent constant $c_{0}$ such that

$$
\begin{aligned}
& \left\|R^{\varepsilon}\right\|_{\varepsilon, p} \leq c_{0}\left\|r^{\varepsilon}\right\|_{\varepsilon, p}, \\
& \left\|S^{\varepsilon}\right\|_{\varepsilon, 3} \leq c_{0} .
\end{aligned}
$$

Proof. We use the linearity to split Eq. (4.1).

Equation on $R^{\varepsilon}$ : Since curl $R^{\varepsilon}=0$, there exists $\varphi^{\varepsilon} \in \mathbf{W}^{1}\left(\mathbb{R}^{2} \times I_{1}\right)$ (Beppo-Levi's space, $c f$. [10]) such that $R^{\varepsilon}=-\nabla \varphi^{\varepsilon}$ and $\varphi^{\varepsilon}$ is solution of:

$$
\begin{cases}-\Delta \varphi^{\varepsilon}=-\operatorname{div} r^{\varepsilon} & \text { in } \omega_{\varepsilon} \\ -\Delta \varphi^{\varepsilon}=0 & \text { in } \omega_{\varepsilon}^{\prime} \\ {\left[-\frac{\partial \varphi^{\varepsilon}}{\partial \nu}+\overline{r^{\varepsilon}} \cdot \nu\right]=0} & \text { on } \partial \omega_{\varepsilon} \\ {\left[\varphi^{\varepsilon}\right]=0} & \text { on } \partial \omega_{\varepsilon}\end{cases}
$$

For all $\Phi, \Psi \in \mathbf{W}^{1}\left(\mathbb{R}^{2} \times I_{1}\right)$, we let $\alpha(\Phi, \Psi)=\int_{\mathbb{R}^{2} \times I_{1}} \nabla \Phi \cdot \nabla \Psi d x$ and

$$
\beta(\Psi)=-\int_{\Omega} \Psi \cdot \operatorname{div} r^{\varepsilon} d x+\int_{\partial \omega_{\varepsilon}}\left(r^{\varepsilon} \cdot \nu\right) \Psi \partial \gamma
$$


The application $\alpha$ is continuous and coercive on $\mathbf{W}^{1}\left(\mathbb{R}^{2} \times I_{1}\right)$ and

$$
|\beta(\Psi)| \leq\left\|r^{\varepsilon}\right\|_{\mathbb{L}^{2}\left(\omega_{\varepsilon}\right)}\|\nabla \Psi\|_{\mathbb{L}_{p}^{2}\left(\mathbb{R}^{2} \times I_{1}\right)} .
$$

We apply now the Lax-Milgram theorem and we obtain the existence of $\varphi^{\varepsilon} \in \mathbf{W}^{1}\left(\mathbb{R}^{2} \times I_{1}\right)$ and $\left\|R^{\varepsilon}\right\|_{\mathbb{L}^{2}\left(\mathbb{R}^{2} \times I_{1}\right)}=\left\|\nabla \varphi^{\varepsilon}\right\|_{\mathbb{L}^{2}\left(\mathbb{R}^{2} \times I_{1}\right)} \leq\left\|r^{\varepsilon}\right\|_{\mathbb{L}^{2}\left(\omega_{\varepsilon}\right)}$. Back to the anisotropic Sobolev norms we have $\left|R^{\varepsilon}\right|_{\varepsilon, 2} \leq\left|r^{\varepsilon}\right|_{\varepsilon, 2}$. To enhance the regularity while taking into account the anisotropy of the spaces, we derive the equation on $R^{\varepsilon}$ in the transversal and longitudinal directions and we reapply the same arguments (we follow the strategy given in $[7,9,14]$ ). We obtain $\left\|R^{\varepsilon}\right\|_{\varepsilon, p} \leq C\left\|r^{\varepsilon}\right\|_{\varepsilon, p}$.

Equation on $S^{\varepsilon}$ : Thanks to the linearity we split again the equation in three terms, one for each one in the second member. We let $S^{\varepsilon}=S^{\varepsilon, 1}+S^{\varepsilon, 2}+S^{\varepsilon, 3}$.

Since the domain $\omega_{\varepsilon}$ is $z$-periodic, we temporarily forget what happens in this direction by decomposing the magnetic field in Fourier series, we are then lead to consider problems of the same kind as the ones solved for $H^{0}, H^{1}, H^{2}$ and $H^{3} . S^{\varepsilon, 1}$ fulfills:

$$
\begin{cases}\operatorname{div} S^{\varepsilon, 1}+\partial_{z} \widetilde{\overline{U_{l}^{3}}}=0 & \text { in } \mathbb{R}^{2} \times I_{1}, \\ \operatorname{curl} S^{\varepsilon, 1}=0 & \text { in } \mathbb{R}^{2} \times I_{1}, \\ {\left[S^{\varepsilon, 1} \cdot \nu\right]=0, \quad\left[S^{\varepsilon, 1} \wedge \nu\right]=0} & \text { on } \partial \omega_{\varepsilon} .\end{cases}
$$

There exists then $\varphi^{\varepsilon, 1}$ such that $S^{\varepsilon, 1}=-\nabla \varphi^{\varepsilon, 1}$, and by decomposing in Fourier series in the $z$ direction the equation we obtain, we get:

$$
-\Delta_{\tau} c_{n}\left(\varphi^{\varepsilon, 1}\right)+n^{2} c_{n}\left(\varphi^{\varepsilon, 1}\right)=i n c_{n}\left(\overline{U_{l}^{3}}\right) \quad \text { in } \mathbb{R}^{2},
$$

where $c_{n}(f)$ is the $n$-th Fourier's coefficient of $f$ and $n \in \mathbb{Z}$. By the same way we used in Prop. 2.3 to obtain the existence and regularity we get that $-\nabla \varphi^{\varepsilon, 1}=S^{\varepsilon, 1} \in \mathbb{L}^{\infty}\left(0, T_{0} ; \mathbb{H}_{\varepsilon}^{4}\right)$ and for all $t<T_{0}$

$$
\left\|S^{\varepsilon, 1}\right\|_{\varepsilon, k} \leq c\left\|U_{l}^{3}\right\|_{\varepsilon, k}, \quad 0 \leq k \leq 4
$$

The application $S^{\varepsilon, 2}$ fulfills:

$$
\begin{cases}\operatorname{div} S^{\varepsilon, 2}+\partial_{z} \widetilde{H_{l}^{3}}=0 & \text { in } \mathbb{R}^{2} \times I_{1}, \\ \operatorname{curl} S^{\varepsilon, 2}=0 & \text { in } \mathbb{R}^{2} \times I_{1}, \\ {\left[S^{\varepsilon, 2} \cdot \nu\right]=0, \quad\left[S^{\varepsilon, 2} \wedge \nu\right]=0} & \text { on } \partial \omega_{\varepsilon} .\end{cases}
$$

In the same way we introduce $\varphi^{\varepsilon, 2}$ such that $S^{\varepsilon, 2}=-\nabla \varphi^{\varepsilon, 2}$ and we use the Fourier series in the $z$-direction. Since $H_{l}^{3}=Q_{l}^{3}$ (cf Th. 2.11), we use the same method as in Prop. 2.4 and we take into account the Fourier variable to get some regularity for $S^{\varepsilon, 1}$. We obtain that $S^{\varepsilon, 2} \in \mathbb{L}^{\infty}\left(0, T_{0} ; \mathbb{H}_{\varepsilon}^{4}\right)$ and for all $0 \leq k \leq 4$,

$$
\left\|S^{\varepsilon, 2}(t, \cdot)\right\|_{\varepsilon, k} \leq c\left(\left\|\partial_{z}^{3} U^{0}\left(t, \frac{\dot{v}}{\varepsilon}, \cdot\right)\right\|_{\varepsilon, k}+\left\|\partial_{z}^{2} U^{1}\left(t, \frac{\dot{\varepsilon}}{\varepsilon}, \cdot\right)\right\|_{\varepsilon, k}+\left\|\partial_{z} U^{2}\left(t, \frac{\dot{-}}{\varepsilon}, \cdot\right)\right\|_{\varepsilon, k}\right) .
$$

The application $S^{\varepsilon, 3}$ fulfills:

$$
\begin{cases}\operatorname{div} S^{\varepsilon, 3}=0 & \text { in } \mathbb{R}^{2} \times I_{1}, \\ \operatorname{curl} S^{\varepsilon, 3}+\partial_{z}\left(e_{3} \wedge \widetilde{H^{3}}\right)= & \text { in } \mathbb{R}^{2} \times I_{1}, \\ {\left[S^{\varepsilon, 3} \cdot \nu\right]=0, \quad\left[S^{\varepsilon, 3} \wedge \nu\right]=0} & \text { on } \partial \omega_{\varepsilon},\end{cases}
$$


which also writes:

$$
\left\{\begin{array}{l}
\operatorname{div}_{\tau} S_{\tau}^{\varepsilon, 3}+\partial_{z} S_{l}^{\varepsilon, 3}=0, \\
\operatorname{curl}_{2 D} S_{\tau}^{\varepsilon, 3}=0, \\
\nabla_{\tau} S_{l}^{\varepsilon, 3}-\partial_{z} S^{\varepsilon, 3}=\partial_{z} H_{\tau}^{3}
\end{array}\right.
$$

Let $\varphi_{l}^{\varepsilon}$ such that $S_{\tau}^{\varepsilon, 3}=-\nabla_{\tau} \varphi_{l}^{\varepsilon}$. Since $H_{\tau}^{3}=-\nabla_{\tau} \varphi^{2}$, we have $S_{l}^{\varepsilon, 3}+\partial_{z} \varphi_{l}^{\varepsilon}=-\partial_{z} \varphi^{3}$ (there is no constant through integration since we assume that there is no external magnetic field), and finally, the divergence equation gives:

$$
-\Delta_{\tau} \varphi_{l}^{\varepsilon}-\partial_{z}^{2} \varphi_{l}^{\varepsilon}=\partial_{z}^{2} \varphi^{3} .
$$

This case is similar to the case of $S^{\varepsilon, 2}$, except that the equation fulfilled by $\varphi^{3}$ is more complicated. Following the proof of Prop. 2.3 and 2.4, the existence proof of $S^{\varepsilon, 1}$ and $S^{\varepsilon, 2}$ and with the results of Prop. 2.1, we obtain that $S^{\varepsilon, 3} \in \mathbb{L}^{\infty}\left(0, T_{0} ; \mathbb{H}_{\varepsilon}^{3}\right)$ and for $0 \leq k \leq 3$,

$$
\left\|S^{\varepsilon, 3}(t, \cdot)\right\|_{\varepsilon, k} \leq C\left(\left\|\partial_{z} U^{3}\right\|_{\varepsilon, k}+\left\|\partial_{z}^{2} U^{2}\right\|_{\varepsilon, k}+\left\|\partial_{z}^{3} U^{1}\right\|_{\varepsilon, k}+\left\|\partial_{z}^{4} U^{1}\right\|_{\varepsilon, k}\right) .
$$

\section{B Expression of $F^{\varepsilon}$}

$$
\begin{aligned}
& F^{\varepsilon}=\varepsilon\left[\partial_{z}^{2} c^{\varepsilon}+\left|\nabla_{\tau} b^{\varepsilon}\right|^{2} c^{\varepsilon}+\varepsilon^{2}\left|\nabla_{\tau} c^{\varepsilon}\right|^{2} c^{\varepsilon}-2 \varepsilon\left|\nabla_{\tau} c^{\varepsilon}\right|^{2} b^{\varepsilon}+\left|\partial_{z} b^{\varepsilon}\right|^{2} a^{\varepsilon}-2 \varepsilon\left|\partial_{z} b^{\varepsilon}\right|^{2} b^{\varepsilon}-2 \varepsilon\left|\nabla_{\tau} b^{\varepsilon}\right|^{2} U^{3}\right. \\
& +\left|\nabla_{\tau} c^{\varepsilon}\right|^{2} a^{\varepsilon}+\left|\partial_{z} a^{\varepsilon}\right|^{2} c^{\varepsilon}+\varepsilon^{2}\left|\partial_{z} b^{\varepsilon}\right|^{2} c^{\varepsilon}+2\left(\nabla_{\tau} U^{3} \cdot \nabla_{\tau} a^{\varepsilon}\right) b^{\varepsilon}+2\left(\partial_{z} a^{\varepsilon} \cdot \partial_{z} b^{\varepsilon}\right) b^{\varepsilon} \\
& +2\left(\nabla_{\tau} U^{3} \cdot \nabla_{\tau} b^{\varepsilon}\right) a^{\varepsilon}+2\left(\nabla_{\tau} a^{\varepsilon} \cdot \nabla_{\tau} b^{\varepsilon}\right) U^{3}-4 \varepsilon\left(\nabla_{\tau} b^{\varepsilon} \cdot \nabla_{\tau} U^{3}\right) b^{\varepsilon}-2 \varepsilon\left(\partial_{z} b^{\varepsilon} \cdot \partial_{z} c^{\varepsilon}\right) a^{\varepsilon} \\
& -2 \varepsilon\left(\nabla_{\tau} c^{\varepsilon} \cdot \nabla_{\tau} U^{3}\right) a^{\varepsilon}+2 \varepsilon^{2}\left(\partial_{z} b^{\varepsilon} \cdot \partial_{z} c^{\varepsilon}\right) b^{\varepsilon}-2 \varepsilon\left(\nabla_{\tau} a^{\varepsilon} \cdot \nabla_{\tau} U^{3}\right) c^{\varepsilon}+2\left(\partial_{z} a^{\varepsilon} \cdot \partial_{z} c^{\varepsilon}\right) a^{\varepsilon} \\
& -2 \varepsilon\left(\partial_{z} a^{\varepsilon} \cdot \partial_{z} b^{\varepsilon}\right) c^{\varepsilon}-2 \varepsilon\left(\partial_{z} a^{\varepsilon} \cdot \partial_{z} c^{\varepsilon}\right) b^{\varepsilon}-2 \varepsilon\left(\nabla_{\tau} a^{\varepsilon} \cdot \nabla_{\tau} c^{\varepsilon}\right) U^{3}+2 \varepsilon^{2}\left(\nabla_{\tau} b^{\varepsilon} \cdot \nabla_{\tau} c^{\varepsilon}\right) U^{3} \\
& +2\left(\nabla_{\tau} a^{\varepsilon} \cdot \nabla_{\tau} c^{\varepsilon}\right) c^{\varepsilon}+2 \varepsilon^{2}\left(\nabla_{\tau} c^{\varepsilon} \cdot \nabla_{\tau} U^{3}\right) b^{\varepsilon}+2 \varepsilon^{2}\left(\nabla_{\tau} b^{\varepsilon} \cdot \nabla_{\tau} U^{3}\right) c^{\varepsilon}-4 \varepsilon\left(\nabla_{\tau} b^{\varepsilon} \cdot \nabla_{\tau} c^{\varepsilon}\right) c^{\varepsilon} \\
& +2\left(\nabla_{\tau} b^{\varepsilon} \cdot \nabla_{\tau} c^{\varepsilon}\right) b^{\varepsilon}+c^{\varepsilon} \wedge \partial_{z}^{2} a^{\varepsilon}+c^{\varepsilon} \wedge \Delta_{\tau} c^{\varepsilon}-\varepsilon c^{\varepsilon} \wedge \partial_{z}^{2} b^{\varepsilon}+a^{\varepsilon} \wedge \partial_{z}^{2} c^{\varepsilon}+b^{\varepsilon} \wedge \partial_{z}^{2} b^{\varepsilon} \\
& +b^{\varepsilon} \wedge \Delta_{\tau} U^{3}+U^{3} \wedge \Delta_{\tau} b^{\varepsilon}-\varepsilon U^{3} \wedge \Delta_{\tau} c^{\varepsilon}-\varepsilon c^{\varepsilon} \wedge \Delta_{\tau} U^{3}-\varepsilon b^{\varepsilon} \wedge \partial_{z}^{2} c^{\varepsilon}+U^{3} \wedge H^{1} \\
& +b^{\varepsilon} \wedge H^{3}+c^{\varepsilon} \wedge H^{2}-U^{3} \wedge\left(a^{\varepsilon} \wedge H^{1}\right)-a^{\varepsilon} \wedge\left(U^{3} \wedge H^{1}\right)-\varepsilon^{4} c^{\varepsilon} \wedge\left(U^{3} \wedge H^{3}\right) \\
& +\varepsilon c^{\varepsilon} \wedge\left(U^{3} \wedge K^{e}\right)+\varepsilon^{3} b^{\varepsilon} \wedge\left(U^{3} \wedge H^{3}\right)-b^{\varepsilon} \wedge\left(a^{\varepsilon} \wedge H^{3}\right)-b^{\varepsilon} \wedge\left(U^{3} \wedge K^{\varepsilon}\right) \\
& -\varepsilon^{4} U^{3} \wedge\left(c^{\varepsilon} \wedge H^{3}\right)+\varepsilon U^{3} \wedge\left(c^{\varepsilon} \wedge K^{e}\right)+\varepsilon^{3} U^{3} \wedge\left(b^{\varepsilon} \wedge H^{3}\right)-U^{3} \wedge\left(b^{\varepsilon} \wedge K^{\varepsilon}\right) \\
& -a^{\varepsilon} \wedge\left(b^{\varepsilon} \wedge H^{3}\right)+\varepsilon^{3} c^{\varepsilon} \wedge\left(c^{\varepsilon} \wedge H^{3}\right)-c^{\varepsilon} \wedge\left(c^{\varepsilon} \wedge K^{\varepsilon}\right)+\varepsilon b^{\varepsilon} \wedge\left(b^{\varepsilon} \wedge H^{3}\right) \\
& -\varepsilon^{2} c^{\varepsilon} \wedge\left(U^{3} \wedge H^{1}\right)-\varepsilon^{3} c^{\varepsilon} \wedge\left(U^{3} \wedge H^{2}\right)-c^{\varepsilon} \wedge\left(a^{\varepsilon} \wedge H^{2}\right)+2 \varepsilon b^{\varepsilon} \wedge\left(U^{3} \wedge H^{1}\right) \\
& +\varepsilon^{2} \beta^{\varepsilon} \wedge\left(U^{3} \wedge H^{2}\right)-\varepsilon^{2} U^{3} \wedge\left(c^{\varepsilon} \wedge H^{1}\right)-\varepsilon^{3} U^{3} \wedge\left(c^{\varepsilon} \wedge H^{2}\right)+a^{\varepsilon} \wedge\left(c^{\varepsilon} \wedge H^{2}\right) \\
& +2 \varepsilon U^{3} \wedge\left(b^{\varepsilon} \wedge H^{1}\right)+\varepsilon^{2} U^{3} \wedge\left(b^{\varepsilon} \wedge H^{2}\right)+\varepsilon^{2} c^{\varepsilon} \wedge\left(c^{\varepsilon} \wedge H^{2}\right)-b^{\varepsilon} \wedge\left(c^{\varepsilon} \wedge H^{1}\right) \\
& +2 \varepsilon c^{\varepsilon} \wedge\left(c^{\varepsilon} \wedge H^{1}\right)-c^{\varepsilon} \wedge\left(b^{\varepsilon} \wedge H^{1}\right)-b^{\varepsilon} \wedge\left(b^{\varepsilon} \wedge H^{2}\right)+\varepsilon b^{\varepsilon} \wedge\left(c^{\varepsilon} \wedge H^{2}\right) \\
& \left.+\varepsilon c^{\varepsilon} \wedge\left(b^{\varepsilon} \wedge H^{2}\right)\right]+a^{\varepsilon} \wedge S^{\varepsilon}-a^{\varepsilon} \wedge\left(a^{\varepsilon} \wedge S^{\varepsilon}\right)
\end{aligned}
$$

\section{Proof of the estimates}

In the following inequalities the operators $\nabla$ and $\Delta$ are the usual differential operators. We then lose some powers in $\varepsilon$ when we perform the estimates in the anisotropic Sobolev spaces:

$$
\begin{aligned}
& \varepsilon|\nabla u|_{\varepsilon, p} \leq\left(\left|\partial_{z} u\right|_{\varepsilon, p}+\left|\varepsilon \nabla_{\tau} u\right|_{\varepsilon, p}\right), \\
& \varepsilon^{2}|\Delta u|_{\varepsilon, p} \leq\left(\left|\partial_{z}^{2} u\right|_{\varepsilon, p}+\left|\varepsilon^{2} \Delta_{\tau} u\right|_{\varepsilon, p}\right) .
\end{aligned}
$$


$\mathbb{L}^{2}$ estimates: we take the scalar product of Eq. (4.2) with $\frac{1}{\left|\omega_{\varepsilon}\right|} r^{\varepsilon}$ to obtain the norm ||$_{\varepsilon, 2}$. The integrations by parts are licit since we consider a Galerkin approximation. We get:

$$
\frac{1}{2} \frac{d}{d t}\left(\left|r^{\varepsilon}\right|_{\varepsilon, 2}^{2}\right)+\varepsilon^{2}\left|\nabla r^{\varepsilon}\right|_{\varepsilon, 2}^{2} \leq \frac{1}{\left|\omega_{\varepsilon}\right|} \int_{\omega_{\varepsilon}}\left(T_{1}+\cdots+T_{8}+F^{\varepsilon}\right) \cdot r^{\varepsilon} d x
$$

We estimate each $\frac{1}{\left|\omega_{\varepsilon}\right|} \int_{\omega_{\varepsilon}} T_{i} r^{\varepsilon} d x$ thanks to Hölder's inequalities then by anisotropic Sobolev's embeddings:

$$
\begin{aligned}
\frac{1}{\left|\omega_{\varepsilon}\right|}\left|\int_{\omega_{\varepsilon}} T_{1} r^{\varepsilon} d x\right| & \leq \varepsilon^{8}\left|r^{\varepsilon}\right|_{\varepsilon, \infty}^{2}\left|\nabla r^{\varepsilon}\right|_{\varepsilon, 2}^{2} \\
& \leq C \varepsilon^{9 / 2}\left|r^{\varepsilon}\right|_{\varepsilon, 2}^{1 / 2}\left\|r^{\varepsilon}\right\|_{\varepsilon, 1}^{2}\left\|\varepsilon r^{\varepsilon}\right\|_{\varepsilon, 2}^{3 / 2} \leq C \varepsilon^{9 / 2} \mathbf{Q}^{2}
\end{aligned}
$$

(thanks to Ineq. (3.5) and Rem. 4.1)

$$
\begin{aligned}
\frac{1}{\left|\omega_{\varepsilon}\right|}\left|\int_{\omega_{\varepsilon}} T_{2} r^{\varepsilon} d x\right| \leq & \varepsilon^{5}\left|a^{\varepsilon}\right|_{\varepsilon, \infty}\left|r^{\varepsilon}\right|_{\varepsilon, 3}\left|\nabla r^{\varepsilon}\right|_{\varepsilon, 3}^{2}+2 \varepsilon^{5}\left|r^{\varepsilon}\right|_{\varepsilon, 3}^{2}\left|\nabla a^{\varepsilon}\right|_{\varepsilon, \infty}\left|\nabla r^{\varepsilon}\right|_{\varepsilon, 3} \\
\leq & C \varepsilon^{2}\left|r^{\varepsilon}\right|_{\varepsilon, 2}^{1 / 2}\left\|r^{\varepsilon}\right\|_{\varepsilon, 1}^{3 / 2}\left\|\varepsilon r^{\varepsilon}\right\|_{\varepsilon, 2}+C \varepsilon^{5 / 2}\left|r^{\varepsilon}\right|_{\varepsilon, 2}\left\|r^{\varepsilon}\right\|_{\varepsilon, 1}^{3 / 2}\left\|\varepsilon r^{\varepsilon}\right\|_{\varepsilon, 2}^{1 / 2} \\
& (\text { thanks to Prop. 4.2, Ineq. (3.4), (3.11) and Rem. 4.1) } \\
\leq & C\left(\varepsilon^{2}+\varepsilon^{5 / 2}\right) \mathbf{Q}^{3 / 2} \\
\frac{1}{\left|\omega_{\varepsilon}\right|}\left|\int_{\omega_{\varepsilon}} T_{3} r^{\varepsilon} d x\right| \leq & \varepsilon^{2}\left|r^{\varepsilon}\right|_{\varepsilon, 2}^{2}\left|\nabla a^{\varepsilon}\right|_{\varepsilon, \infty}^{2}+2 \varepsilon^{2}\left|a^{\varepsilon}\right|_{\varepsilon, \infty}\left|r^{\varepsilon}\right|_{\varepsilon, 2}\left|\nabla r^{\varepsilon}\right|_{\varepsilon, 2}\left|\nabla a^{\varepsilon}\right|_{\varepsilon, \infty} \\
\leq & C\left|r^{\varepsilon}\right|_{\varepsilon, 2}^{2}+C\left|r^{\varepsilon}\right|_{\varepsilon, 2}\left\|r^{\varepsilon}\right\|_{\varepsilon, 1} \leq C \mathbf{Q}
\end{aligned}
$$

(thanks to Prop. 4.2 and Rem. 4.1)

$$
\begin{aligned}
\frac{1}{\left|\omega_{\varepsilon}\right|}\left|\int_{\omega_{\varepsilon}} T_{4} r^{\varepsilon} d x\right| & \leq \varepsilon^{2}\left|r^{\varepsilon}\right|_{\varepsilon, 2}\left|\nabla a^{\varepsilon}\right|_{\varepsilon, \infty}\left|\nabla r^{\varepsilon}\right|_{\varepsilon, 2} \\
& \leq C\left|r^{\varepsilon}\right|_{\varepsilon, 2}\left\|r^{\varepsilon}\right\|_{\varepsilon, 1} \leq C \mathbf{Q}
\end{aligned}
$$

(thanks to Prop. 4.2 and Rem. 4.1)

$$
\begin{aligned}
\frac{1}{\left|\omega_{\varepsilon}\right|}\left|\int_{\omega_{\varepsilon}} T_{5} r^{\varepsilon} d x\right| & \leq\left|a^{\varepsilon}\right|_{\varepsilon, \infty}\left|r^{\varepsilon}\right|_{\varepsilon, 2}\left|R^{\varepsilon}\right|_{\varepsilon, 2} \\
& \leq C\left|r^{\varepsilon}\right|_{\varepsilon, 2}^{2} \leq C \mathbf{Q}
\end{aligned}
$$

(thanks to Prop. 4.2 and Th. 4.1) 


$$
\begin{aligned}
\frac{1}{\left|\omega_{\varepsilon}\right|}\left|\int_{\omega_{\varepsilon}} T_{6} r^{\varepsilon} d x\right| & \leq\left|a^{\varepsilon}\right|_{\varepsilon, \infty}^{2}\left|r^{\varepsilon}\right|_{\varepsilon, 2}\left|R^{\varepsilon}\right|_{\varepsilon, 2}+\left|a^{\varepsilon}\right|_{\varepsilon, \infty}\left|K^{\varepsilon}\right|_{\varepsilon, \infty}\left|r^{\varepsilon}\right|_{\varepsilon, 2}^{2} \\
& \leq C\left|r^{\varepsilon}\right|_{\varepsilon, 2}^{2} \leq C \mathbf{Q}
\end{aligned}
$$

(thanks to Prop. 4.2 and Th. 4.1)

$$
\begin{aligned}
\frac{1}{\left|\omega_{\varepsilon}\right|}\left|\int_{\omega_{\varepsilon}} T_{7} r^{\varepsilon} d x\right| & \leq C \varepsilon^{3}\left|a^{\varepsilon}\right|_{\varepsilon, \infty}\left|r^{\varepsilon}\right|_{\varepsilon, 4}^{2}\left|R^{\varepsilon}\right|_{\varepsilon, 2} \\
& \leq C \varepsilon^{3}\left|r^{\varepsilon}\right|_{\varepsilon, 2}^{3 / 2}\left\|r^{\varepsilon}\right\|_{\varepsilon, 1}^{3 / 2} \leq C \varepsilon^{3} \mathbf{Q}^{3 / 2}
\end{aligned}
$$

(thanks to Prop. 4.2, Ineq. (3.4) and Th. 4.1)

$$
\begin{aligned}
\frac{1}{\left|\omega_{\varepsilon}\right|}\left|\int_{\omega_{\varepsilon}} T_{8} r^{\varepsilon} d x\right|= & 0 \\
\frac{1}{\left|\omega_{\varepsilon}\right|}\left|\int_{\omega_{\varepsilon}} F^{\varepsilon} r^{\varepsilon} d x\right| & \leq\left|F^{\varepsilon}\right|_{\varepsilon, 2}\left|r^{\varepsilon}\right|_{\varepsilon, 2} \\
& \leq C\left(1+\left|r^{\varepsilon}\right|_{\varepsilon, 2}^{2}\right)
\end{aligned}
$$

(thanks to Prop. 4.2 and Young's inequality)

By summing the previous inequalities we obtain the existence of a constant $C$ and a polynomial $P$, both independent from $\varepsilon$ such that:

$$
\frac{d}{d t}\left|r^{\varepsilon}\right|_{\varepsilon, 2}^{2}+\varepsilon^{2}\left|\nabla r^{\varepsilon}\right|_{\varepsilon, 2}^{2} \leq C(1+\mathbf{Q})+\varepsilon^{2} P(\mathbf{Q}),
$$

$\mathbb{H}^{1}$ estimates: By taking the scalar product of the Galerkin approximation of Eq. (4.2) by

$$
\frac{1}{\left|\omega_{\varepsilon}\right|}\left(\partial_{z}^{2} r^{\varepsilon}+\varepsilon^{2} \Delta_{\tau} r^{\varepsilon}\right)
$$

and by integrating by parts we get:

$$
\begin{array}{r}
\frac{1}{2} \frac{d}{d t}\left(\left|\partial_{z} r^{\varepsilon}\right|_{\varepsilon, 2}^{2}+\left|\varepsilon \nabla_{\tau} r^{\varepsilon}\right|_{\varepsilon, 2}^{2}\right)+\varepsilon^{2}\left|\partial_{z}^{2} r^{\varepsilon}\right|_{\varepsilon, 2}^{2}+\left|\varepsilon^{2} \Delta_{\tau} r^{\varepsilon}\right|_{\varepsilon, 2}^{2}+\varepsilon^{2}\left|\nabla_{\tau} \partial_{z} r^{\varepsilon}\right|_{\varepsilon, 2}^{2}+\varepsilon^{4}\left|\nabla_{\tau} \partial_{z} r^{\varepsilon}\right|_{\varepsilon, 2}^{2} \\
\leq \frac{1}{\left|\omega_{\varepsilon}\right|} \int_{\omega_{\varepsilon}}\left(T_{1}+\cdots+T_{8}+F^{\varepsilon}\right) \cdot\left(\partial_{z}^{2} r^{\varepsilon}+\varepsilon^{2} \Delta_{\tau} r^{\varepsilon}\right) d x
\end{array}
$$

and we estimate each term of the second member in the following way:

$$
\begin{aligned}
\frac{1}{\left|\omega_{\varepsilon}\right|}\left|\int_{\omega_{\varepsilon}} T_{1}\left(\partial_{z}^{2} r^{\varepsilon}+\varepsilon^{2} \Delta_{\tau} r^{\varepsilon}\right) d x\right| & \leq \varepsilon^{8}\left|r^{\varepsilon}\right|_{\varepsilon, 6}\left|\nabla r^{\varepsilon}\right|_{\varepsilon, 6}^{2}\left(\left|\partial_{z}^{2} r^{\varepsilon}\right|_{\varepsilon, 2}+\left|\varepsilon^{2} \Delta_{\tau} r^{\varepsilon}\right|_{\varepsilon, 2}\right) \\
& \leq C \varepsilon^{3}\left\|r^{\varepsilon}\right\|_{\varepsilon, 1}\left\|\varepsilon r^{\varepsilon}\right\|_{\varepsilon, 2}^{3} \leq C \varepsilon^{3} \mathbf{Q}^{2}
\end{aligned}
$$

(thanks to Ineq. (3.4), (3.11) and Rem. 4.1)

$$
\begin{aligned}
\frac{1}{\left|\omega_{\varepsilon}\right|}\left|\int_{\omega_{\varepsilon}} T_{2}\left(\partial_{z}^{2} r^{\varepsilon}+\varepsilon^{2} \Delta_{\tau} r^{\varepsilon}\right) d x\right| \leq & \varepsilon^{5}\left|a^{\varepsilon}\right|_{\varepsilon, \infty}\left|\nabla r^{\varepsilon}\right|_{\varepsilon, 4}^{2}\left(\left|\partial_{z}^{2} r^{\varepsilon}\right|_{\varepsilon, 2}+\left|\varepsilon^{2} \Delta_{\tau} r^{\varepsilon}\right|_{\varepsilon, 2}\right) \\
& +2 \varepsilon^{5}\left|r^{\varepsilon}\right|_{\varepsilon, 6}\left|\nabla r^{\varepsilon}\right|_{\varepsilon, 3}\left|\nabla a^{\varepsilon}\right|_{\varepsilon, \infty}\left(\left|\partial_{z}^{2} r^{\varepsilon}\right|_{\varepsilon, 2}+\left|\varepsilon^{2} \Delta_{\tau} r^{\varepsilon}\right|_{\varepsilon, 2}\right) \\
\leq & C \varepsilon^{1 / 2}\left\|r^{\varepsilon}\right\|_{\varepsilon, 1}^{1 / 2}\left\|\varepsilon r^{\varepsilon}\right\|_{\varepsilon, 2}^{5 / 2}+C \varepsilon^{3 / 2}\left\|r^{\varepsilon}\right\|_{\varepsilon, 1}^{3 / 2}\left\|\varepsilon r^{\varepsilon}\right\|_{\varepsilon, 2}^{3 / 2} \\
& (\text { thanks to Prop. } 4.2, \text { Ineq. (3.4), (3.11) and Rem. 4.1) } \\
\leq & C\left(\varepsilon^{1 / 2}+\varepsilon^{3 / 2}\right) \mathbf{Q}^{3 / 2}
\end{aligned}
$$




$$
\begin{aligned}
\frac{1}{\left|\omega_{\varepsilon}\right|}\left|\int_{\omega_{\varepsilon}} T_{3}\left(\partial_{z}^{2} r^{\varepsilon}+\varepsilon^{2} \Delta_{\tau} r^{\varepsilon}\right) d x\right| \leq & \varepsilon^{2}\left|\nabla a^{\varepsilon}\right|_{\varepsilon, \infty}^{2}\left\|r^{\varepsilon}\right\|_{\varepsilon, 1}^{2} \\
& +2 \varepsilon^{2}\left|a^{\varepsilon}\right|_{\varepsilon, \infty}\left(\left|\partial_{z} \nabla a^{\varepsilon}\right|_{\varepsilon, \infty}+\left|\varepsilon \nabla_{\tau} \nabla a^{\varepsilon}\right|_{\varepsilon, \infty}\right)\left|r^{\varepsilon}\right|_{\varepsilon, 2}\left\|r^{\varepsilon}\right\|_{\varepsilon, 1} \\
& +2 \varepsilon^{2}\left|\nabla a^{\varepsilon}\right|_{\varepsilon, \infty}\left(\left|\partial_{z} a^{\varepsilon}\right|_{\varepsilon, \infty}+\left|\varepsilon \nabla_{\tau} a^{\varepsilon}\right|_{\varepsilon, \infty}\right)\left|\nabla r^{\varepsilon}\right|_{\varepsilon, 2}\left\|r^{\varepsilon}\right\|_{\varepsilon, 1} \\
& +2 \varepsilon^{2}\left|a^{\varepsilon}\right|_{\varepsilon, \infty}\left(\left|\partial_{z} \nabla a^{\varepsilon}\right|_{\varepsilon, \infty}+\left|\varepsilon \nabla \nabla_{\tau} \nabla a^{\varepsilon}\right|_{\varepsilon, \infty}\right)\left|r^{\varepsilon}\right|_{\varepsilon, 2}\left\|r^{\varepsilon}\right\|_{\varepsilon, 1} \\
& +2 \varepsilon^{2}\left|a^{\varepsilon}\right|_{\varepsilon, \infty}\left|\nabla a^{\varepsilon}\right|_{\varepsilon, \infty}\left(\left|\partial_{z}^{2} r^{\varepsilon}\right|_{\varepsilon, 2}+\left|\varepsilon \partial_{z} \nabla_{\tau} r^{\varepsilon}\right|_{\varepsilon, 2}\right. \\
& \left.+\left|\partial_{z} \nabla_{\tau} r^{\varepsilon}\right|_{\varepsilon, 2}+\left|\varepsilon \Delta_{\tau} r^{\varepsilon}\right|_{\varepsilon, 2}\right)\left\|r^{\varepsilon}\right\|_{\varepsilon, 1} \\
\leq & C\left(\left\|r^{\varepsilon}\right\|_{\varepsilon, 1}^{2}+\left|r^{\varepsilon}\right|_{\varepsilon, 2}\left\|r^{\varepsilon}\right\|_{\varepsilon, 1}\right) \\
& +C\left\|r^{\varepsilon}\right\|_{\varepsilon, 1}\left(\varepsilon\left|\partial_{z}^{2} r^{\varepsilon}\right|_{\varepsilon, 2}+(1+\varepsilon)\left|\varepsilon \nabla r_{\tau} \nabla r^{\varepsilon}\right|_{\varepsilon, 2}+\left|\varepsilon^{2} \Delta_{\tau} r^{\varepsilon}\right|_{\varepsilon, 2}\right)
\end{aligned}
$$

(thanks to Prop. 4.2 and Rem. 4.1)

$$
\leq C \mathbf{Q}+\frac{1}{18}\left(\varepsilon^{2}\left|\partial_{z}^{2} r^{\varepsilon}\right|_{\varepsilon, 2}^{2}+\left(1+\varepsilon^{2}\right)\left|\varepsilon \nabla_{\tau} \nabla r^{\varepsilon}\right|_{\varepsilon, 2}^{2}+\left|\varepsilon^{2} \Delta_{\tau} r^{\varepsilon}\right|_{\varepsilon, 2}^{2}\right)
$$

To estimate $T_{4}$ we look more precisely the behaviour of the terms with $\Delta r^{\varepsilon}$ thanks to integrations by parts (which are licit thanks to the splitting between the two directions):

$$
\begin{aligned}
A_{1} & =\frac{1}{\left|\omega_{\varepsilon}\right|} \int_{\omega_{\varepsilon}} \varepsilon^{2} a^{\varepsilon} \wedge \Delta r^{\varepsilon} \cdot\left(\partial_{z}^{2} r^{\varepsilon}+\varepsilon^{2} \Delta_{\tau} r^{\varepsilon}\right) d x \\
& =\frac{1}{\left|\omega_{\varepsilon}\right|} \int_{\omega_{\varepsilon}}^{\varepsilon^{2}}\left(a^{\varepsilon} \wedge \partial_{z}^{2} r^{\varepsilon} \cdot \varepsilon^{2} \Delta_{\tau} r^{\varepsilon}+a^{\varepsilon} \wedge \Delta_{\tau} r^{\varepsilon} \cdot \partial_{z}^{2} r^{\varepsilon}\right) d x \\
& =\frac{1-\varepsilon^{2}}{\left|\omega_{\varepsilon}\right|}\left(\int_{\omega_{\varepsilon}}-\varepsilon^{2} \partial_{z} a^{\varepsilon} \wedge \Delta_{\tau} r^{\varepsilon} \cdot \partial_{z} r^{\varepsilon} d x+\int_{\omega_{\varepsilon}} \varepsilon^{2} \nabla_{\tau} a^{\varepsilon} \wedge \nabla_{\tau} \partial_{z} r^{\varepsilon} \cdot \partial_{z} r^{\varepsilon} d x\right) \\
& \leq\left|\partial_{z} a^{\varepsilon}\right|_{\varepsilon, \infty}\left\|r^{\varepsilon}\right\|_{\varepsilon, 1}\left|\varepsilon^{2} \Delta_{\tau} r^{\varepsilon}\right|_{\varepsilon, 2}+\left|\varepsilon \nabla_{\tau} a^{\varepsilon}\right|_{\varepsilon, \infty}\left\|r^{\varepsilon}\right\|_{\varepsilon, 1}\left|\varepsilon \partial_{z} \nabla_{\tau} r^{\varepsilon}\right|_{\varepsilon, 2} \\
& \leq C\left\|r^{\varepsilon}\right\|_{\varepsilon, 1}^{2}+\frac{\varepsilon^{2}}{18}\left(\left|\varepsilon^{2} \Delta_{\tau} r^{\varepsilon}\right|_{\varepsilon, 2}^{2}+\left|\varepsilon \partial_{z} \nabla_{\tau} r^{\varepsilon}\right|_{\varepsilon, 2}^{2}\right) \\
A_{2} & =\frac{1}{\left|\omega_{\varepsilon}\right|} \int_{\omega_{\varepsilon}} \varepsilon^{2} r^{\varepsilon} \wedge \Delta r^{\varepsilon} \cdot\left(\partial_{z}^{2} r^{\varepsilon}+\varepsilon^{2} \Delta_{\tau} r^{\varepsilon}\right) d x \\
& =\frac{1}{\left|\omega_{\varepsilon}\right|} \int_{\omega_{\varepsilon}}^{\varepsilon^{5}}\left(r^{\varepsilon} \wedge \partial_{z}^{2} r^{\varepsilon} \cdot \varepsilon^{2} \Delta_{\tau} r^{\varepsilon}+r^{\varepsilon} \wedge \Delta_{\tau} r^{\varepsilon} \cdot \partial_{z}^{2} r^{\varepsilon}\right) d x \\
& =\frac{1-\varepsilon^{2}}{\left|\omega_{\varepsilon}\right|} \int_{\omega_{\varepsilon}} \varepsilon^{5} \partial_{z} r^{\varepsilon} \wedge \nabla_{\tau} r^{\varepsilon} \cdot \partial_{z} \nabla_{\tau} r^{\varepsilon} d x \\
& \leq C \varepsilon^{3}\left|\partial_{z} r^{\varepsilon}\right|_{\varepsilon, 4}\left|\varepsilon \nabla_{\tau} r^{\varepsilon}\right|_{\varepsilon, 4}\left|\varepsilon \partial_{z} \nabla_{\tau} r^{\varepsilon}\right|_{\varepsilon, 2} .
\end{aligned}
$$

Back in the estimates of $T_{4}$ we obtain:

$$
\begin{aligned}
\frac{1}{\left|\omega_{\varepsilon}\right|}\left|\int_{\omega_{\varepsilon}} T_{4}\left(\partial_{z}^{2} r^{\varepsilon}+\varepsilon^{2} \Delta_{\tau} r^{\varepsilon}\right) d x\right| \leq & \varepsilon^{2}\left|\Delta a^{\varepsilon}\right|_{\varepsilon, \infty}\left\|r^{\varepsilon}\right\|_{\varepsilon, 1}^{2} \\
& +\varepsilon^{2}\left(\left|\partial_{z} \Delta a^{\varepsilon}\right|_{\varepsilon, 3}+\left|\varepsilon \nabla_{\tau} \Delta a^{\varepsilon}\right|_{\varepsilon, 3}\right)\left|r^{\varepsilon}\right|_{\varepsilon, 6}\left\|r^{\varepsilon}\right\|_{\varepsilon, 1} \\
\leq & C\left\|r^{\varepsilon}\right\|_{\varepsilon, 1}^{2}+C \varepsilon^{1 / 2}\left\|r^{\varepsilon}\right\|_{\varepsilon, 1}^{1 / 2}\left\|\varepsilon r^{\varepsilon}\right\|_{\varepsilon, 2}^{3 / 2}\left(\varepsilon\left|\varepsilon \partial_{z} \nabla_{\tau} r^{\varepsilon}\right|_{\varepsilon, 2}\right) \\
& +\frac{1}{18}\left(\left|\varepsilon^{2} \Delta_{\tau} r^{\varepsilon}\right|_{\varepsilon, 2}^{2}+\left|\varepsilon \partial_{z} \nabla_{\tau} r^{\varepsilon}\right|_{\varepsilon, 2}^{2}\right) \\
& (\text { thanks to Prop. } 4.2, \text { Ineq. (3.4) and Rem. 4.1) } \\
\leq & C \mathbf{Q}+C \varepsilon^{1 / 2} \mathbf{Q}^{3 / 2}+\frac{1}{18}\left(\left|\varepsilon^{2} \Delta_{\tau} r^{\varepsilon}\right|_{\varepsilon, 2}^{2}+\left|\varepsilon \partial_{z} \nabla_{\tau} r^{\varepsilon}\right|_{\varepsilon, 2}^{2}\right)
\end{aligned}
$$




$$
\begin{aligned}
& \frac{1}{\left|\omega_{\varepsilon}\right|}\left|\int_{\omega_{\varepsilon}} T_{5}\left(\partial_{z}^{2} r^{\varepsilon}+\varepsilon^{2} \Delta_{\tau} r^{\varepsilon}\right) d x\right| \leq\left\|a^{\varepsilon}\right\|_{\mathbb{W} \mathbb{E}^{1, \infty}}\left|R^{\varepsilon}\right|_{\varepsilon, 2}\left\|r^{\varepsilon}\right\|_{\varepsilon, 1}+\left|a^{\varepsilon}\right|_{\varepsilon, \infty}\left\|R^{\varepsilon}\right\|_{\varepsilon, 1}\left\|r^{\varepsilon}\right\|_{\varepsilon, 1} \\
& +\left\|K^{\varepsilon}\right\|_{\mathbb{W}_{\varepsilon}^{1, \infty}}\left|r^{\varepsilon}\right|_{\varepsilon, 2}\left\|r^{\varepsilon}\right\|_{\varepsilon, 1} \\
& +\varepsilon^{3}\left|r^{\varepsilon}\right|_{\varepsilon, 6}\left\|R^{\varepsilon}\right\|_{\varepsilon, 1}\left(\left|\partial_{z} r^{\varepsilon}\right|_{\varepsilon, 3}+\left|\varepsilon \nabla_{\tau} r^{\varepsilon}\right|_{\varepsilon, 3}\right) \\
& \leq C\left\|r^{\varepsilon}\right\|_{\varepsilon, 1}^{2}+C \varepsilon^{5 / 2}\left\|r^{\varepsilon}\right\|_{\varepsilon, 1}^{5 / 2}\left\|\varepsilon r^{\varepsilon}\right\|_{\varepsilon, 2}^{1 / 2} \\
& \text { (thanks to Prop. 4.2, Th. } 4.1 \text { and Ineq. 3.4) } \\
& \leq C \mathbf{Q}+C \varepsilon^{5 / 2} \mathbf{Q}^{3 / 2} \\
& \frac{1}{\left|\omega_{\varepsilon}\right|}\left|\int_{\omega_{\varepsilon}} T_{6}\left(\partial_{z}^{2} r^{\varepsilon}+\varepsilon^{2} \Delta_{\tau} r^{\varepsilon}\right) d x\right| \leq 2\left\|a^{\varepsilon}\right\|_{\mathbb{W}_{\varepsilon}^{1, \infty}}\left|a^{\varepsilon}\right|_{\varepsilon, \infty}\left|R^{\varepsilon}\right|_{\varepsilon, 2}\left\|r^{\varepsilon}\right\|_{\varepsilon, 1}+\left|a^{\varepsilon}\right|_{\varepsilon, \infty}^{2}\left\|R^{\varepsilon}\right\|_{\varepsilon, 1}\left\|r^{\varepsilon}\right\|_{\varepsilon, 1} \\
& +2\left\|a^{\varepsilon}\right\|_{\mathbb{W}_{\varepsilon}^{1, \infty}}\left|K^{\varepsilon}\right|_{\varepsilon, \infty}\left|r^{\varepsilon}\right|_{\varepsilon, 2}\left\|r^{\varepsilon}\right\|_{\varepsilon, 1} \\
& +2\left\|\left.K^{\varepsilon}\right|_{\mathbb{W}_{\varepsilon}^{1, \infty}}\left|a^{\varepsilon}\right|_{\varepsilon, \infty}\left|r^{\varepsilon}\right|_{\varepsilon, 2}|| r^{\varepsilon}\right\|_{\varepsilon, 1} \\
& +\left|a^{\varepsilon}\right|_{\varepsilon, \infty}\left|K^{\varepsilon}\right|_{\varepsilon, \infty}\left\|r^{\varepsilon}\right\|_{\varepsilon, 1}^{2} \\
& \leq C\left(\left|r^{\varepsilon}\right|_{\varepsilon, 2}+\left\|r^{\varepsilon}\right\|_{\varepsilon, 1}\right)\left\|r^{\varepsilon}\right\|_{\varepsilon, 1} \leq C \mathbf{Q} \\
& \text { (thanks to Prop. } 4.2 \text { and Th. 4.1) }
\end{aligned}
$$$$
\begin{aligned}
\frac{1}{\left|\omega_{\varepsilon}\right|}\left|\int_{\omega_{\varepsilon}} T_{7}\left(\partial_{z}^{2} r^{\varepsilon}+\varepsilon^{2} \Delta_{\tau} r^{\varepsilon}\right) d x\right| \leq & \varepsilon^{3}\left(\left|r^{\varepsilon}\right|_{\varepsilon, 4}^{2}\left|K^{\varepsilon}\right|_{\varepsilon, \infty}+2\left|a^{\varepsilon}\right|_{\varepsilon, \infty}\left|r^{\varepsilon}\right|_{\varepsilon, 4}\left|R^{\varepsilon}\right|_{\varepsilon, 4}\right) \\
& \times\left(\left|\partial_{z}^{2} r^{\varepsilon}\right|_{\varepsilon, 2}+\left|\varepsilon^{2} \Delta_{\tau} r^{\varepsilon}\right|_{\varepsilon, 2}\right) \\
\leq & C \varepsilon^{2}\left|r^{\varepsilon}\right|_{\varepsilon, 2}^{1 / 2}\left\|r^{\varepsilon}\right\|_{\varepsilon, 1}^{3 / 2}\left\|\varepsilon r^{\varepsilon}\right\|_{\varepsilon, 2} \leq C \varepsilon^{2} \mathbf{Q}^{3 / 2}
\end{aligned}
$$

(thanks to Prop. 4.2, Th. 4.1 and Ineq. (3.4))

$$
\begin{aligned}
\frac{1}{\left|\omega_{\varepsilon}\right|}\left|\int_{\omega_{\varepsilon}} T_{8}\left(\partial_{z}^{2} r^{\varepsilon}+\varepsilon^{2} \Delta_{\tau} r^{\varepsilon}\right) d x\right| \leq & \varepsilon^{6}\left|r^{\varepsilon}\right|_{\varepsilon, 6}^{2}\left|R^{\varepsilon}\right|_{\varepsilon, 6}\left(\left|\partial_{z}^{2} r^{\varepsilon}\right|_{\varepsilon, 2}+\left|\varepsilon^{2} \Delta_{\tau} r^{\varepsilon}\right|_{\varepsilon, 2}\right) \\
\leq & C \varepsilon^{5}\left\|r^{\varepsilon}\right\|_{\varepsilon, 1}^{3}\left\|\varepsilon r^{\varepsilon}\right\|_{\varepsilon, 2} \leq C \varepsilon^{5} \mathbf{Q}^{2} \\
& \text { (thanks to Th. 4.1 and Ineq. (3.4)) }
\end{aligned}
$$

$$
\begin{aligned}
\frac{1}{\left|\omega_{\varepsilon}\right|}\left|\int_{\omega_{\varepsilon}} F^{\varepsilon}\left(\partial_{z}^{2} r^{\varepsilon}+\varepsilon^{2} \Delta_{\tau} r^{\varepsilon}\right) d x\right| & \leq\left\|F^{\varepsilon}\right\|_{\varepsilon, 1}\left\|r^{\varepsilon}\right\|_{\varepsilon, 1} \\
& \leq C(1+\mathbf{Q})
\end{aligned}
$$

(thanks to Prop. 4.2 and Young's Inequality)

By summing the previous estimates and absorbing the terms that appeared during the estimate of $T_{4}$, we get:

$$
\begin{aligned}
\frac{1}{2} \frac{d}{d t}\left(\left|\partial_{z} r^{\varepsilon}\right|_{\varepsilon, 2}^{2}+\left|\varepsilon \nabla_{\tau} r^{\varepsilon}\right|_{\varepsilon, 2}^{2}\right)+\frac{1}{2}\left(\varepsilon^{2}\left|\partial_{z}^{2} \varepsilon^{\varepsilon}\right|_{\varepsilon, 2}^{2}+\left|\varepsilon^{2} \Delta_{\tau} r^{\varepsilon}\right|_{\varepsilon, 2}^{2}\right. & \left.+\left(1+\varepsilon^{2}\right)\left|\varepsilon \partial_{z} \nabla_{\tau} r^{\varepsilon}\right|_{\varepsilon, 2}^{2}\right) \\
& \leq C(1+\mathbf{Q})+\varepsilon^{2} P(\mathbf{Q}),
\end{aligned}
$$

where $C$ is a constant and $P$ a polynomial, both independent from $\varepsilon$.

$\mathbb{H}^{2}$ estimates: We now estimate $\varepsilon^{2}\left(\left|\partial_{z}^{2} r^{\varepsilon}\right|_{\varepsilon, 2}^{2}+\left|\varepsilon \partial_{z} \nabla_{\tau} r^{\varepsilon}\right|_{\varepsilon, 2}+\left|\varepsilon^{2} \Delta_{\tau} r^{\varepsilon}\right|_{\varepsilon, 2}^{2}\right)$ by taking the scalar product of (4.2) by $\frac{\varepsilon^{2}}{\left|\omega_{\varepsilon}\right|}\left(\partial_{z}^{4}+\varepsilon^{2} \partial_{z}^{2} \Delta_{\tau}+\varepsilon^{4} \Delta_{\tau}^{2}\right) r^{\varepsilon}=\frac{\varepsilon^{2}}{\left|\omega_{\varepsilon}\right|} \mathbf{B}$. For each term we perform an integration by parts, use Hölder's inequality, anisotropic Sobolev embeddings and finally Young's inequality to isolate the term

$$
\varepsilon^{4} \mathbf{A}^{2}=\varepsilon^{4}\left|\partial_{z}^{3} r^{\varepsilon}\right|_{\varepsilon, 2}^{2}+\varepsilon^{2}\left|\varepsilon \partial_{z}^{2} \nabla_{\tau} r^{\varepsilon}\right|_{\varepsilon, 2}^{2}+\varepsilon^{2}\left|\varepsilon^{2} \partial_{z} \Delta_{\tau} r^{\varepsilon}\right|_{\varepsilon, 2}^{2}+\varepsilon^{2}\left|\varepsilon^{3} \nabla_{\tau} \Delta_{\tau} r^{\varepsilon}\right|_{\varepsilon, 2}^{2} .
$$


We finally have:

$$
\begin{aligned}
\frac{1}{\left|\omega_{\varepsilon}\right|}\left|\int_{\omega_{\varepsilon}} \varepsilon^{2} T_{1} \mathbf{B} d x\right| \leq & \varepsilon^{10}\left|\nabla r^{\varepsilon}\right|_{\varepsilon, 6}^{2}\left(2\left|\partial_{z} r^{\varepsilon}\right|_{\varepsilon, 6}+\left|\varepsilon \nabla_{\tau} r^{\varepsilon}\right|_{\varepsilon, 6}\right) \mathbf{A} \\
& +\varepsilon^{10}\left|r^{\varepsilon}\right|_{\varepsilon, 12}\left(2\left|\partial_{z} \nabla r^{\varepsilon}\right|_{\varepsilon, 3}+\left|\varepsilon \nabla_{\tau} \nabla r^{\varepsilon}\right|_{\varepsilon, 3}\right)\left|\nabla r^{\varepsilon}\right|_{\varepsilon, 12} \mathbf{A} \\
\leq & C \varepsilon^{8}\left\|r^{\varepsilon}\right\|_{\varepsilon, 1}^{3 / 4}\left\|r^{\varepsilon}\right\|_{\varepsilon, 2}^{3 / 2}\left(\left\|r^{\varepsilon}\right\|_{\varepsilon, 2}^{1 / 4}+\mathbf{A}^{1 / 4}\right)\left(\left\|r^{\varepsilon}\right\|_{\varepsilon, 2}^{1 / 2}+\mathbf{A}^{1 / 2}\right) \mathbf{A} \\
& +C \varepsilon^{5} \mathbf{A}\left\|\varepsilon r^{\varepsilon}\right\|_{\varepsilon, 2}^{3}
\end{aligned}
$$

(thanks to Ineq. (3.4), (3.6), (3.11), (3.12) and Rem. 4.1)

$$
\leq \frac{1}{18} \varepsilon^{4} \mathbf{A}^{2}+C \varepsilon^{6} \mathbf{Q}^{3}+C \varepsilon^{28 / 3} \mathbf{Q}^{11 / 3}+C \varepsilon^{13} \mathbf{Q}^{5}+C \varepsilon^{24} \mathbf{Q}^{9}
$$

$$
\begin{aligned}
\frac{1}{\left|\omega_{\varepsilon}\right|}\left|\int_{\omega_{\varepsilon}} \varepsilon^{2} T_{2} \mathbf{B} d x\right| \leq & \varepsilon^{7}\left(2\left|\partial_{z} a^{\varepsilon}\right|_{\varepsilon, \infty}+\left|\varepsilon \nabla_{\tau} a^{\varepsilon}\right|_{\varepsilon, \infty}\right)\left|\nabla r^{\varepsilon}\right|_{\varepsilon, 4}^{2} \mathbf{A} \\
& +2 \varepsilon^{7}\left|a^{\varepsilon}\right|_{\varepsilon, \infty}\left|\nabla r^{\varepsilon}\right|_{\varepsilon, 6}\left(2\left|\partial_{z} \nabla r^{\varepsilon}\right|_{\varepsilon, 3}+\left|\varepsilon \nabla_{\tau} \nabla r^{\varepsilon}\right|_{\varepsilon, 3}\right) \mathbf{A} \\
& +2 \varepsilon^{7}\left|\nabla a^{\varepsilon}\right|_{\varepsilon, \infty}\left|\nabla r^{\varepsilon}\right|_{\varepsilon, 4}\left(2\left|\partial_{z} r^{\varepsilon}\right|_{\varepsilon, 4}+\left|\varepsilon \nabla_{\tau} r^{\varepsilon}\right|_{\varepsilon, 4}\right) \mathbf{A} \\
& +2 \varepsilon^{7}\left(2\left|\partial_{z} \nabla a^{\varepsilon}\right|_{\varepsilon, \infty}+\left|\varepsilon \nabla_{\tau} a^{\varepsilon}\right|_{\varepsilon, \infty}\right)\left|r^{\varepsilon}\right|_{\varepsilon, 6}\left|\nabla r^{\varepsilon}\right|_{\varepsilon, 3} \mathbf{A} \\
& +2 \varepsilon^{7}\left|\nabla a^{\varepsilon}\right|_{\varepsilon, \infty}\left|r^{\varepsilon}\right|_{\varepsilon, 6}\left(2\left|\partial_{z} \nabla r^{\varepsilon}\right|_{\varepsilon, 3}+\left|\varepsilon \nabla_{\tau} \nabla r^{\varepsilon}\right|_{\varepsilon, 3}\right) \mathbf{A} \\
\leq & C \varepsilon^{7 / 2}\left\|r^{\varepsilon}\right\|_{\varepsilon, 1}^{1 / 2}\left\|\varepsilon r^{\varepsilon}\right\|_{\varepsilon, 2}^{3 / 2} \mathbf{A}+C \varepsilon^{5}\left\|r^{\varepsilon}\right\|_{\varepsilon, 2}^{3 / 2}\left(\left\|r^{\varepsilon}\right\|_{\varepsilon, 2}^{1 / 2}+\mathbf{A}^{1 / 2}\right) \mathbf{A} \\
& +C \varepsilon^{7 / 2}\left\|r^{\varepsilon}\right\|_{\varepsilon, 1}^{1 / 2}\left\|\varepsilon r^{\varepsilon}\right\|_{\varepsilon, 2}^{3 / 2} \mathbf{A}+C \varepsilon^{9 / 2}\left\|r^{\varepsilon}\right\|_{\varepsilon, 1}^{3 / 2}\left\|\varepsilon r^{\varepsilon}\right\|_{\varepsilon, 2}^{1 / 2} \mathbf{A} \\
& +C \varepsilon^{5}\left\|r^{\varepsilon}\right\|_{\varepsilon, 1}\left\|r^{\varepsilon}\right\|_{\varepsilon, 2}^{1 / 2}\left(\left\|r^{\varepsilon}\right\|_{\varepsilon, 2}^{1 / 2}+\mathbf{A}^{1 / 2}\right) \mathbf{A}
\end{aligned}
$$

(thanks to Ineq. (3.4), (3.11), (3.5), Prop. 4.2 and Rem. 4.1)

$$
\leq \frac{1}{18} \varepsilon^{4} \mathbf{A}^{2}+C \varepsilon^{2} \mathbf{Q}^{2}+C \varepsilon^{2} \mathbf{Q}^{3}
$$

To obtain the estimates of $T_{3}$ we integrate by parts several times the term:

$$
\begin{aligned}
A=\frac{2 \varepsilon^{4}}{\left|\omega_{\varepsilon}\right|} \int_{\omega_{\varepsilon}}\left(\nabla_{\tau} a^{\varepsilon} \cdot \partial_{z} \nabla_{\tau} r^{\varepsilon}\right)\left(a^{\varepsilon} \cdot \partial_{z}^{3} r^{\varepsilon}\right) d x \leq & 2 \varepsilon^{4}\left|\Delta_{\tau} a^{\varepsilon}\right|_{\varepsilon, \infty}\left|a^{\varepsilon}\right|_{\varepsilon, \infty}\left|\partial_{z} r^{\varepsilon}\right|_{\varepsilon, 2}\left|\partial_{z}^{3} r^{\varepsilon}\right|_{\varepsilon, 2} \\
& +2 \varepsilon^{4}\left|\nabla_{\tau} a^{\varepsilon}\right|_{\varepsilon, \infty}^{2}\left|\partial_{z} r^{\varepsilon}\right|_{\varepsilon, 2}\left|\partial_{z}^{3} r^{\varepsilon}\right|_{\varepsilon, 2}\left|\partial_{\tau}^{2} \nabla_{\tau} r^{\varepsilon}\right|_{\varepsilon, 2} \\
& +2 \varepsilon^{4}\left|a^{\varepsilon}\right|_{\varepsilon, \infty}\left|\partial_{z} \nabla_{\tau} a^{\varepsilon}\right|_{\varepsilon, \infty}\left|\partial_{z} r^{\varepsilon}\right|_{\varepsilon, 2}\left|\partial_{z}^{2} \nabla_{\tau} r^{\varepsilon}\right|_{\varepsilon, 2} \\
& +2 \varepsilon^{4}\left|\nabla_{\tau} a^{\varepsilon}\right|_{\varepsilon, \infty}\left|\partial_{z} a^{\varepsilon}\right|_{\varepsilon, \infty}\left|\partial_{z} r^{\varepsilon}\right|_{\varepsilon, 2}\left|\partial_{z}^{2} \nabla^{2}\right|_{\varepsilon, 2} \\
& +2 \varepsilon^{4}\left|\nabla_{\tau} a^{\varepsilon}\right|_{\varepsilon, \infty}\left|a^{\varepsilon}\right|_{\varepsilon, \infty}\left|\partial_{z}^{2} r^{\varepsilon}\right|_{\varepsilon, 2}\left|\partial_{z}^{2} \nabla_{\tau} r^{\varepsilon}\right|_{\varepsilon, 2} \\
\leq & C \mathbf{Q}^{1 / 2}\left(\varepsilon^{2} \mathbf{A}\right)
\end{aligned}
$$


Back in the estimate of $T_{3}$,

$$
\begin{aligned}
\frac{1}{\left|\omega_{\varepsilon}\right|}\left|\int_{\omega_{\varepsilon}} \varepsilon^{2} T_{3} \mathbf{B} d x\right| \leq & \varepsilon^{4}\left|\nabla a^{\varepsilon}\right|_{\varepsilon, \infty}^{2}\left(\left|\partial_{z} r^{\varepsilon}\right|_{\varepsilon, 2}\left|\partial_{z}^{3} r^{\varepsilon}\right|_{\varepsilon, 2}+\left|\partial_{z} r^{\varepsilon}\right|_{\varepsilon, 2}\left|\varepsilon^{2} \partial_{z} \Delta_{\tau} r^{\varepsilon}\right|_{\varepsilon, 2}\right. \\
& \left.+\left|\varepsilon \nabla_{\tau} r^{\varepsilon}\right|_{\varepsilon, 2}\left|\varepsilon^{3} \nabla_{\tau} \Delta_{\tau} r^{\varepsilon}\right|_{\varepsilon, 2}\right)+2 \varepsilon^{4}\left|\nabla a^{\varepsilon}\right|_{\varepsilon, \infty}\left|r^{\varepsilon}\right|_{\varepsilon, 2}\left(\left|\partial_{z} \nabla a^{\varepsilon}\right|_{\varepsilon, \infty}\left|\partial_{z}^{3} r^{\varepsilon}\right|_{\varepsilon, 2}\right. \\
& \left.+\left|\partial_{z} \nabla a^{\varepsilon}\right|_{\varepsilon, \infty}\left|\varepsilon^{2} \partial_{z} \Delta_{\tau} r^{\varepsilon}\right|_{\varepsilon, 2}+\left|\varepsilon \nabla_{\tau} \nabla a^{\varepsilon}\right|_{\varepsilon, \infty}\left|\varepsilon^{3} \nabla_{\tau} \Delta_{\tau} r^{\varepsilon}\right|_{\varepsilon, 2}\right) \\
& +2 \varepsilon^{4}\left|\nabla a^{\varepsilon}\right|_{\varepsilon, \infty}\left|\nabla r^{\varepsilon}\right|_{\varepsilon, 2}\left(\left|\partial_{z} a^{\varepsilon}\right|_{\varepsilon, \infty}\left|\partial_{z}^{3} r^{\varepsilon}\right|_{\varepsilon, 2}\right. \\
& \left.+\left|\partial_{z} a^{\varepsilon}\right|_{\varepsilon, \infty}\left|\varepsilon^{2} \partial_{z} \Delta_{\tau} r^{\varepsilon}\right|_{\varepsilon, 2}+\left|\varepsilon \nabla_{\tau} a^{\varepsilon}\right|_{\varepsilon, \infty}\left|\varepsilon^{3} \nabla_{\tau} \Delta_{\tau} r^{\varepsilon}\right|_{\varepsilon, 2}\right) \\
& +2 \varepsilon^{4}\left|a^{\varepsilon}\right|_{\varepsilon, \infty}\left|\nabla r^{\varepsilon}\right|_{\varepsilon, 2}\left(\left|\partial_{z} \nabla a^{\varepsilon}\right|_{\varepsilon, \infty}\left|\partial_{z}^{3} r^{\varepsilon}\right|_{\varepsilon, 2}+\left|\partial_{z} \nabla a^{\varepsilon}\right|_{\varepsilon, \infty}\left|\varepsilon^{2} \partial_{z} \Delta_{\tau} r^{\varepsilon}\right|_{\varepsilon, 2}\right. \\
& \left.+\left|\varepsilon \nabla_{\tau} \nabla a^{\varepsilon}\right|_{\varepsilon, \infty}\left|\varepsilon^{3} \nabla_{\tau} \Delta_{\tau} r^{\varepsilon}\right|_{\varepsilon, 2}\right)+2 \varepsilon^{4}\left|a^{\varepsilon}\right|_{\varepsilon, \infty}\left|\nabla a^{\varepsilon}\right|_{\varepsilon, \infty}\left(\left|\partial_{z}^{2} r^{\varepsilon}\right|_{\varepsilon, 2}\left|\partial_{z}^{3} r^{\varepsilon}\right|_{\varepsilon, 2}\right. \\
& \left.+\left|\partial_{z} \nabla r^{\varepsilon}\right|_{\varepsilon, 2}\left|\varepsilon^{2} \partial_{z} \Delta_{\tau} r^{\varepsilon}\right|_{\varepsilon, 2}+\left|\varepsilon \nabla_{\tau} \nabla r^{\varepsilon}\right|_{\varepsilon, 2}\left|\varepsilon^{3} \nabla_{\tau} \Delta_{\tau} r^{\varepsilon}\right|_{\varepsilon, 2}\right)+A \\
\leq & C\left(\left\|r^{\varepsilon}\right\|_{\varepsilon, 1}+\left\|\varepsilon r^{\varepsilon}\right\|_{\varepsilon, 2}\right)\left(\varepsilon^{2} \mathbf{A}\right) \\
& (\text { thanks to Ineq. (3.4), (3.11), Prop. 4.2 and Rem. 4.1) } \\
\leq & \frac{1}{18} \varepsilon^{4} \mathbf{A}^{4}+C \mathbf{Q}
\end{aligned}
$$

To obtain the estimates of $T_{4}$, we integrate by parts several times each terms:

$$
\begin{aligned}
A & =\frac{\varepsilon^{4}}{\left|\omega_{\varepsilon}\right|} \int_{\omega_{\varepsilon}} r^{\varepsilon} \wedge \Delta a^{\varepsilon} \cdot\left(\partial_{z}^{4} r^{\varepsilon}+\varepsilon^{2} \partial_{z}^{2} \Delta_{\tau} r^{\varepsilon}+\varepsilon^{4} \Delta_{\tau}^{2} r^{\varepsilon}\right) d x \\
& \leq\left|\Delta a^{\varepsilon}\right|_{\varepsilon, \infty}\left(\left|\partial_{z} r^{\varepsilon}\right|_{\varepsilon, 2}\left|\partial_{z}^{3} r^{\varepsilon}\right|_{\varepsilon, 2}+\left|\partial_{z} r^{\varepsilon}\right|_{\varepsilon, 2}\left|\varepsilon^{2} \partial_{z} \Delta_{\tau} r^{\varepsilon}\right|_{\varepsilon, 2}+\left|\varepsilon \nabla_{\tau} r^{\varepsilon}\right|_{\varepsilon, 2}\left|\varepsilon^{3} \nabla_{\tau} \Delta_{\tau} r^{\varepsilon}\right|_{\varepsilon, 2}\right) \\
B & =\frac{\varepsilon^{4}}{\left|\omega_{\varepsilon}\right|} \int_{\omega_{\varepsilon}} a^{\varepsilon} \wedge\left(\partial_{z}^{2} r^{\varepsilon}+\Delta_{\tau} r^{\varepsilon}\right) \cdot\left(\partial_{z}^{4} r^{\varepsilon}+\varepsilon^{2} \partial_{z}^{2} \Delta_{\tau} r^{\varepsilon}+\varepsilon^{4} \Delta_{\tau}^{2} r^{\varepsilon}\right) d x \\
& =B_{1}+B_{2}+B_{3}+B_{4}+B_{5}+B_{6},
\end{aligned}
$$

where

$$
\begin{aligned}
B_{1}=\frac{\varepsilon^{4}}{\left|\omega_{\varepsilon}\right|} \int_{\omega_{\varepsilon}} a^{\varepsilon} \wedge \partial_{z}^{2} r^{\varepsilon} \cdot \partial_{z}^{4} r^{\varepsilon} d x \leq & \varepsilon^{4}\left|\partial_{z} a^{\varepsilon}\right|_{\varepsilon, \infty}\left|\partial_{z}^{2} r^{\varepsilon}\right|_{\varepsilon, 2}\left|\partial_{z}^{3} r^{\varepsilon}\right|_{\varepsilon, 2} \\
\leq & \frac{1}{200} \varepsilon^{4} \mathbf{A}^{2}+C \varepsilon^{2}\left\|\varepsilon r^{\varepsilon}\right\|_{\varepsilon, 2}^{2} \\
B_{2}=\frac{\varepsilon^{4}}{\left|\omega_{\varepsilon}\right|} \int_{\omega_{\varepsilon}} a^{\varepsilon} \wedge \Delta_{\tau} r^{\varepsilon} \cdot \partial_{z}^{2} r^{\varepsilon} d x= & \frac{\varepsilon^{4}}{\left|\omega_{\varepsilon}\right|} \int_{\omega_{\varepsilon}} \partial_{z} a^{\varepsilon} \wedge \Delta_{\tau} r^{\varepsilon} \cdot \partial_{z}^{3} r^{\varepsilon} d x \\
& +\frac{\varepsilon^{4}}{\left|\omega_{\varepsilon}\right|} \int_{\omega_{\varepsilon}} a^{\varepsilon} \wedge \partial_{z} \Delta_{\tau} r^{\varepsilon} \cdot \partial_{z}^{3} r^{\varepsilon} d x \\
\leq & \left.\varepsilon^{4}\left|\partial_{z} \nabla_{\tau} a_{\varepsilon, \infty}\right| \nabla_{\tau} r^{\varepsilon}\right|_{\varepsilon, 2}\left|\partial_{z}^{3} r^{\varepsilon}\right|_{\varepsilon, 2} \\
& +\varepsilon^{4}\left|\partial_{z}^{2} a^{\varepsilon}\right|_{\varepsilon, \infty}\left|\nabla_{\tau} r^{\varepsilon}\right|_{\varepsilon, 2}\left|\partial_{z}^{2} \nabla_{\tau} r^{\varepsilon}\right|_{\varepsilon, 2} \\
& +\varepsilon^{4}\left|\partial_{z} a^{\varepsilon}\right|_{\varepsilon, \infty}\left|\partial_{z} \nabla_{\tau} r^{\varepsilon}\right|_{\varepsilon, 2}\left|\partial_{z}^{2} \nabla_{\tau} r^{\varepsilon}\right|_{\varepsilon, 2} \\
& +\varepsilon^{4}\left|\nabla_{\tau} a^{\varepsilon}\right|_{\varepsilon, \infty}\left|\partial_{z} \nabla_{\tau} r^{\varepsilon}\right|_{\varepsilon, 2}\left|\partial_{z}^{3} r^{\varepsilon}\right|_{\varepsilon, 2} \\
& +\varepsilon^{4}\left|\partial_{z} a^{\varepsilon}\right|_{\varepsilon, \infty}\left|\partial_{z} \nabla_{\tau} r^{\varepsilon}\right|_{\varepsilon, 2}\left|\partial_{z}^{2} \nabla_{\tau} r^{\varepsilon}\right|_{\varepsilon, 2} \\
\leq & \frac{1}{200} \varepsilon^{4} \mathbf{A}^{2}+C \mathbf{Q}
\end{aligned}
$$




$$
\begin{aligned}
B_{3}=\frac{\varepsilon^{6}}{\left|\omega_{\varepsilon}\right|} \int_{\omega_{\varepsilon}} a^{\varepsilon} \wedge \partial_{z}^{2} r^{\varepsilon} \cdot \partial_{z}^{2} \Delta_{\tau} r^{\varepsilon} d x \leq & \varepsilon^{6}\left|\nabla_{\tau} a^{\varepsilon}\right|_{\varepsilon, \infty}\left|\partial_{z}^{2} r^{\varepsilon}\right|_{\varepsilon, 2}\left|\partial_{z}^{2} \nabla_{\tau} r^{\varepsilon}\right|_{\varepsilon, 2} \\
\leq & \frac{1}{200} \varepsilon^{4} \mathbf{A}^{2}+C \varepsilon^{4} \mathbf{Q} \\
B_{4}=\frac{\varepsilon^{6}}{\left|\omega_{\varepsilon}\right|} \int_{\omega_{\varepsilon}} a^{\varepsilon} \wedge \Delta_{\tau} r^{\varepsilon} \cdot \partial_{z}^{2} \Delta_{\tau} r^{\varepsilon} d x \leq & \left|\partial_{z} a^{\varepsilon}\right|_{\varepsilon, \infty}\left|\Delta_{\tau} r^{\varepsilon}\right|_{\varepsilon, 2}\left|\partial_{z} \Delta_{\tau} r^{\varepsilon}\right|_{\varepsilon, 2} \\
\leq & \frac{1}{200} \varepsilon^{4} \mathbf{A}^{2}+C \mathbf{Q} \\
B_{5}=\frac{\varepsilon^{8}}{\left|\omega_{\varepsilon}\right|} \int_{\omega_{\varepsilon}} a^{\varepsilon} \wedge \partial_{z}^{2} r^{\varepsilon} \cdot \Delta_{\tau}^{2} r^{\varepsilon} d x \leq & \varepsilon^{8}\left|\nabla_{\tau} a^{\varepsilon}\right|_{\varepsilon, \infty}\left|\partial_{z}^{2} r^{\varepsilon}\right|_{\varepsilon, 2}\left|\nabla_{\tau} \Delta_{\tau} r^{\varepsilon}\right|_{\varepsilon, 2} \\
& +\varepsilon^{8}\left|\partial_{z} a^{\varepsilon}\right|_{\varepsilon, \infty}\left|\partial_{z} \nabla_{\tau} r^{\varepsilon}\right|_{\varepsilon, 2}\left|\nabla_{\tau} \Delta_{\tau} r^{\varepsilon}\right|_{\varepsilon, 2} \\
& +\varepsilon^{8}\left|\nabla_{\tau} a^{\varepsilon}\right|_{\varepsilon, \infty}\left|\partial_{z} \nabla_{\tau} r^{\varepsilon}\right|_{\varepsilon, 2}\left|\partial_{z} \Delta_{\tau} r^{\varepsilon}\right|_{\varepsilon, 2} \\
\leq & \frac{1}{200} \varepsilon^{4} \mathbf{A}^{2}+C \varepsilon^{4} \mathbf{Q} \\
B_{6}=\frac{\varepsilon^{8}}{\left|\omega_{\varepsilon}\right|} \int_{\omega_{\varepsilon}} a^{\varepsilon} \wedge \Delta_{\tau} r^{\varepsilon} \cdot \Delta_{\tau}^{2} r^{\varepsilon} d x \leq & \varepsilon^{8}\left|\nabla_{\tau} a^{\varepsilon}\right|_{\varepsilon, \infty}\left|\Delta_{\tau} r^{\varepsilon}\right|_{\varepsilon, 2}\left|\nabla_{\tau} \Delta_{\tau} r^{\varepsilon}\right|_{\varepsilon, 2} \\
\leq & \frac{1}{200} \mathbf{A}+C \varepsilon^{2}\left\|\varepsilon r^{\varepsilon}\right\|_{\varepsilon, 2}^{2} .
\end{aligned}
$$

By the same way,

$$
\begin{aligned}
C= & \frac{\varepsilon^{7}}{\left|\omega_{\varepsilon}\right|} \int_{\omega_{\varepsilon}} r^{\varepsilon} \wedge\left(\partial_{z}^{2} r^{\varepsilon}+\Delta_{\tau} r^{\varepsilon}\right) \cdot\left(\partial_{z}^{4} r^{\varepsilon}+\varepsilon^{4} \Delta_{\tau}^{2} r^{\varepsilon}\right) d x \\
\leq & 2 \varepsilon^{7}\left(\left|\partial_{z} r^{\varepsilon}\right|_{\varepsilon, 6}+\left|\varepsilon \nabla_{\tau} r^{\varepsilon}\right|_{\varepsilon, 6}\right)\left|\Delta r^{\varepsilon}\right|_{\varepsilon, 3} \mathbf{A}+\varepsilon^{7}\left|\nabla_{\tau} r^{\varepsilon}\right|_{\varepsilon, 6}\left|\nabla_{\tau} \partial_{z} r^{\varepsilon}\right|_{\varepsilon, 3}\left|\partial_{z}^{3} r^{\varepsilon}\right|_{\varepsilon, 2} \\
& +\varepsilon^{7}\left|\partial_{z} r^{\varepsilon}\right|_{\varepsilon, 6}\left|\nabla_{\tau} \partial_{z} r^{\varepsilon}\right|_{\varepsilon, 3}\left|\partial_{z}^{2} \nabla_{\tau} r^{\varepsilon}\right|_{\varepsilon, 2}+\varepsilon^{11}\left|\partial_{z} r^{\varepsilon}\right|_{\varepsilon, 6}\left|\nabla_{\tau} \partial_{z} r^{\varepsilon}\right|_{\varepsilon, 3}\left|\nabla_{\tau} \Delta_{\tau} r^{\varepsilon}\right|_{\varepsilon, 2} \\
& +\varepsilon^{11}\left|\nabla_{\tau} r^{\varepsilon}\right|_{\varepsilon, 6}\left|\nabla_{\tau} \partial_{z} r^{\varepsilon}\right|_{\varepsilon, 3}\left|\partial_{z} \Delta_{\tau} r^{\varepsilon}\right|_{\varepsilon, 2} \\
\leq & C \varepsilon^{3} \mathbf{Q} \mathbf{A}+C \varepsilon^{7 / 2} \mathbf{Q}^{3 / 4} \mathbf{A}^{3 / 2} \leq \frac{1}{200} \varepsilon^{4} \mathbf{A}^{2}+C \varepsilon^{2} \mathbf{Q}^{2}+C \varepsilon^{2} \mathbf{Q}^{3}
\end{aligned}
$$

Back in the estimate of $T_{4}$,

$$
\begin{aligned}
& \frac{1}{\left|\omega_{\varepsilon}\right|}\left|\int_{\omega_{\varepsilon}} \varepsilon^{2} T_{4} \mathbf{B} d x\right| \leq A+B+C \\
& \leq \frac{1}{18} \varepsilon^{4} \mathbf{A}^{2}+C \mathbf{Q}+C \varepsilon^{2} \mathbf{Q}^{2}+C \varepsilon^{2} \mathbf{Q}^{3} \\
& \frac{1}{\left|\omega_{\varepsilon}\right|}\left|\int_{\omega_{\varepsilon}} \varepsilon^{2} T_{5} \mathbf{B} d x\right| \leq \varepsilon^{2}\left\|r^{\varepsilon}\right\|_{\varepsilon, 1}\left|K^{\varepsilon}\right|_{\varepsilon, \infty} \mathbf{A}+\varepsilon^{2}\left|r^{\varepsilon}\right|_{\varepsilon, 2}\left\|K^{\varepsilon}\right\|_{\mathbb{W}_{\varepsilon}^{1, \infty}} \mathbf{A} \\
& +\varepsilon^{2}\left\|a^{\varepsilon}\right\|_{\mathbb{W}_{\varepsilon}^{1, \infty}}\left|R^{\varepsilon}\right|_{\varepsilon, 2} \mathbf{A}+\varepsilon^{2}\left|a^{\varepsilon}\right|_{\varepsilon, \infty}\left\|R^{\varepsilon}\right\|_{\varepsilon, 1} \mathbf{A} \\
& +\varepsilon^{5}\left(\left|\partial_{z} r^{\varepsilon}\right|_{\varepsilon, 3}+\left|\varepsilon \nabla_{\tau} r^{\varepsilon}\right|_{\varepsilon, 3}\right)\left|R^{\varepsilon}\right|_{\varepsilon, 6} \mathbf{A} \\
& +\varepsilon^{5}\left|r^{\varepsilon}\right|_{\varepsilon, 6}\left(\left|\partial_{z} R^{\varepsilon}\right|_{\varepsilon, 3}+\left|\varepsilon \nabla_{\tau} R^{\varepsilon}\right|_{\varepsilon, 3}\right) \mathbf{A} \\
& \leq C \varepsilon^{2}\left\|r^{\varepsilon}\right\|_{\varepsilon, 1} \mathbf{A}+C \varepsilon^{5}\left\|r^{\varepsilon}\right\|_{\varepsilon, 1}\left\|r^{\varepsilon}\right\|_{\varepsilon, 2}^{1 / 2}\left(\left\|r^{\varepsilon}\right\|_{\varepsilon, 2}^{1 / 2}+\mathbf{A}^{1 / 2}\right) \mathbf{A} \\
& \text { (thanks to Ineq. (3.4), Prop. 4.2, Th. } 4.1 \text { and Rem. 4.1) } \\
& \leq C \mathbf{Q}^{1 / 2}\left(\varepsilon^{2} \mathbf{A}\right)+C \varepsilon^{2} \mathbf{Q}\left(\varepsilon^{2} \mathbf{A}\right)+C \varepsilon^{3 / 2} \mathbf{Q}^{3 / 4}\left(\varepsilon^{2} \mathbf{A}\right)^{3 / 2} \\
& \leq \frac{1}{18} \varepsilon^{4} \mathbf{A}^{2}+C \mathbf{Q}+C \varepsilon^{4} \mathbf{Q}^{2}+C \varepsilon^{6} \mathbf{Q}^{3}
\end{aligned}
$$




$$
\begin{aligned}
\frac{1}{\left|\omega_{\varepsilon}\right|}\left|\int_{\omega_{\varepsilon}} \varepsilon^{2} T_{6} \mathbf{B} d x\right| \leq & 2 \varepsilon^{2}\left\|a^{\varepsilon}\right\|_{\mathbb{W}_{\varepsilon}^{1, \infty}}\left|a^{\varepsilon}\right|_{\varepsilon, \infty}\left|r^{\varepsilon}\right|_{\varepsilon, 2} \mathbf{A}+\varepsilon^{2}\left|a^{\varepsilon}\right|_{\varepsilon, \infty}^{2}\left\|r^{\varepsilon}\right\|_{\varepsilon, 1} \mathbf{A} \\
& +2 \varepsilon^{2}\left\|a^{\varepsilon}\right\|_{\mathbb{W}_{\varepsilon}^{1, \infty}}\left|K^{\varepsilon}\right|_{\varepsilon, \infty}\left|r^{\varepsilon}\right|_{\varepsilon, 2} \mathbf{A} \\
& +2 \varepsilon^{2}\left\|K^{\varepsilon}\right\|_{\mathbb{W}_{\varepsilon}^{1, \infty}\left|a^{\varepsilon}\right|_{\varepsilon, \infty}\left|r^{\varepsilon}\right|_{\varepsilon, 2} \mathbf{A}} \\
& +2 \varepsilon^{2}\left|a^{\varepsilon}\right|_{\varepsilon, \infty}\left|K^{\varepsilon}\right|_{\varepsilon, \infty}\left\|r^{\varepsilon}\right\|_{\varepsilon, 1} \mathbf{A} \\
\leq & C \varepsilon^{2}\left\|r^{\varepsilon}\right\|_{\varepsilon, 1} \mathbf{A} \leq \frac{1}{18} \varepsilon^{4} \mathbf{A}^{2}+C \mathbf{Q}
\end{aligned}
$$

(thanks to Prop. 4.2 and Th. 4.1)

$$
\begin{aligned}
& \frac{1}{\left|\omega_{\varepsilon}\right|}\left|\int_{\omega_{\varepsilon}} \varepsilon^{2} T_{7} \mathbf{B} d x\right| \leq 2 \varepsilon^{5}\left|a^{\varepsilon}\right|_{\varepsilon, \infty}\left|r^{\varepsilon}\right|_{\varepsilon, 6}\left(\left|\partial_{z} R^{\varepsilon}\right|_{\varepsilon, 3}+\left|\varepsilon \nabla_{\tau} R^{\varepsilon}\right|_{\varepsilon, 3}\right) \mathbf{A} \\
& +2 \varepsilon^{5}\left|a^{\varepsilon}\right|_{\varepsilon, \infty}\left|R^{\varepsilon}\right|_{\varepsilon, 6}\left(\left|\partial_{z} r^{\varepsilon}\right|_{\varepsilon, 3}+\left|\varepsilon \nabla_{\tau} r^{\varepsilon}\right|_{\varepsilon, 3}\right) \mathbf{A} \\
& +2 \varepsilon^{5}\left\|a^{\varepsilon}\right\|_{\mathbb{W}_{\varepsilon}^{1, \infty}}\left|r^{\varepsilon}\right|_{\varepsilon, 4}\left|R^{\varepsilon}\right|_{\varepsilon, 4} \mathbf{A} \\
& +2 \varepsilon^{5}\left|K^{\varepsilon}\right|_{\varepsilon, \infty}\left|r^{\varepsilon}\right|_{\varepsilon, 6}\left(\left|\partial_{z} r^{\varepsilon}\right|_{\varepsilon, 3}+\left|\varepsilon \nabla_{\tau} r^{\varepsilon}\right|_{\varepsilon, 3}\right) \mathbf{A} \\
& +\varepsilon^{5}\left\|K^{\varepsilon}\right\|_{\mathbb{W}^{1, \infty}}\left|r^{\varepsilon}\right|_{\varepsilon, 4}^{2} \mathbf{A} \\
& \leq C \varepsilon^{5 / 2}\left\|r^{\varepsilon}\right\|_{\varepsilon, 1}^{3 / 2}\left\|\varepsilon r^{\varepsilon}\right\|_{\varepsilon, 2}^{1 / 2}\left(\varepsilon^{2} \mathbf{A}\right)+C \varepsilon^{3}\left|r^{\varepsilon}\right|_{\varepsilon, 2}^{1 / 2}\left\|r^{\varepsilon}\right\|_{\varepsilon, 1}^{3 / 2}\left(\varepsilon^{2} \mathbf{A}\right) \\
& \text { (thanks to Ineq. (3.4), Prop. } 4.2 \text { and Th. 4.1) } \\
& \leq \frac{1}{18} \varepsilon^{2} \mathbf{A}^{4}+C\left(\varepsilon^{5}+\varepsilon^{6}\right) \mathbf{Q}^{2} \\
& \frac{1}{\left|\omega_{\varepsilon}\right|}\left|\int_{\omega_{\varepsilon}} \varepsilon^{2} T_{8} \mathbf{B} d x\right| \leq 2 \varepsilon^{8}\left|r^{\varepsilon}\right|_{\varepsilon, 6}\left(\left|\partial_{z} r^{\varepsilon}\right|_{\varepsilon, 6}+\left|\varepsilon \nabla_{\tau} r^{\varepsilon}\right|_{\varepsilon, 6}\right)\left|R^{\varepsilon}\right|_{\varepsilon, 6} \mathbf{A} \\
& +\varepsilon^{8}\left|r^{\varepsilon}\right|_{\varepsilon, 6}^{2}\left(\left|\partial_{z} R^{\varepsilon}\right|_{\varepsilon, 6}+\left|\varepsilon \nabla_{\tau} R^{\varepsilon}\right|_{\varepsilon, 6}\right) \mathbf{A} \\
& \leq C \varepsilon^{5}\left\|r^{\varepsilon}\right\|_{\varepsilon, 1}^{2}\left\|\varepsilon r^{\varepsilon}\right\|_{\varepsilon, 2}\left(\varepsilon^{2} \mathbf{A}\right)
\end{aligned}
$$

(thanks to Ineq. (3.4) and Th. 4.1)

$$
\leq \frac{1}{18} \varepsilon^{4} \mathbf{A}^{2}+C \varepsilon^{10} \mathbf{Q}^{3}
$$

$$
\begin{aligned}
\frac{1}{\left|\omega_{\varepsilon}\right|}\left|\int_{\omega_{\varepsilon}} \varepsilon^{2} F^{\varepsilon} \mathbf{B} d x\right| & \leq\left\|F^{\varepsilon}\right\|_{\varepsilon, 1}\left(\varepsilon^{2} \mathbf{A}\right) \\
& \leq \frac{1}{18} \varepsilon^{4} \mathbf{A}^{2}+C \\
& \text { (thanks to Prop. 4.2) }
\end{aligned}
$$

By summing the previous inequalities we get:

$$
\frac{d}{d t}\left(\varepsilon^{2}\left|\partial_{z}^{2} r^{\varepsilon}\right|_{\varepsilon, 2}^{2}+\varepsilon^{2}\left|\varepsilon \partial_{z} \nabla_{\tau} r^{\varepsilon}\right|_{\varepsilon, 2}^{2}+\varepsilon^{2}\left|\varepsilon^{2} \Delta_{\tau} r^{\varepsilon}\right|_{\varepsilon, 2}^{2}\right)+\varepsilon^{4} \mathbf{A}^{2} \leq C(1+\mathbf{Q})+\varepsilon^{2} P(\mathbf{Q}),
$$

where $P$ is an $\varepsilon$-independent polynomial.

By summing the inequalities (C.1), (C.2) and (C.3) we finally obtain:

$$
\frac{d}{d t} \mathbf{Q} \leq C(1+\mathbf{Q})+\varepsilon^{2} P(\mathbf{Q}),
$$

wich implies the inequality (4.3) and then the main theorems. 


\section{References}

[1] R.A. Adams, Sobolev Spaces, Pure and Applied Math., Vol 65, Academic press, 1975.

[2] P. Bolley and J. Camus, Quelques résultats sur les espaces de Sobolev avec poids, Publication des séminaires de mathématiques, Rennes, 1968-1969.

[3] H. Brézis, Analyse fonctionnelle, Masson, 1993.

[4] W.F. Brown, Micromagnetics, Interscience Publishers, 1963.

[5] V. Bruneau, G. Carbou, Spectral asymptotic in the large coupling limit, Asymptotic Anal. $\mathbf{2 9}(2)$ (2002), 91-113.

[6] G. Carbou, Thin layers in micromagnetism, M3AS, Vol. 11, No. 9 (2001) 1529-1546.

[7] G. Carbou, P. Fabrie, Regular Solutions for Landau-Lifschitz Equation in a Bounded domain, Differential and Integral Equations, 14 (2001), 213-229.

[8] G. Carbou, P. Fabrie, Time average in Micromagnetism, Journal of Differential Equations 147 (1998), 383-409.

[9] G. Carbou, P. Fabrie, O. Guès, Couche limite dans un modèle de ferromagnétisme, Commun. Partial Differ. Equations, Vol. 27, No. 7-8 (2002), 1467-1495.

[10] R. Dautray et J.L. Lions, Analyse mathématique et calcul numérique pour les sciences et les techniques, Tomes 1, 2 et 3, Coll. du C.E.A., Masson.

[11] C. Foias, R. Temam, Remarque sur les équations de Navier-Stokes stationnaires et les phénomènes successifs de bifurcation, Ann. Sc. Norm. Super. Pisa, Cl. Sci., IV. Ser. 5 (1978), 29-63.

[12] E. Grenier, O. Guès, Boundary layers for viscous perturbations of noncharacteristic quasilinear hyperbolic problems, Journal of Differential Equations 143 (1998), 110-146.

[13] H. Haddar, Modèle asymptotique en ferromagnétisme: Couches minces et homogénéisation, Thesis (2000), ENPC.

[14] O.A. Ladyzhenskaya, The boundary value problems of mathematical physics, SpringerVerlag Applied Math. Sciences, Vol. 49, 1985.

[15] L. Landau and E. Lifshitz, On the theory of magnetic permeability in ferromagnetic body, Physik. Z. Soviet Union 8 (1935) 153-169.

[16] M.N. Le Roux, Méthode d'éléments finis pour la résolution numérique de problèmes extérieurs en dimension 2, RAIRO Anal. Numér. 11 (1977), no. 1, 27-60.

[17] J.L. Lions, Quelques méthodes de résolution des problèmes aux limites non linéaires, Gauthier-Villars, 1969.

[18] W. McLean, Strongly Elliptic Systems and Boundary Integral Equations, Cambridge University Press, 2000.

[19] J. Necas, Les méthodes directes en théorie des équations elliptiques, Masson, 1967. 
[20] J.C. Nédélec, Acoustic and Electromagnetic Equations- Integral Representations for Harmonic Problems, Applied Mathematical Sciences 144, Springer, 2001.

[21] D. Sanchez, Phénomène de couche limite dans un modèle de ferromagnétisme, Ann. Fac. Sci. Toulouse VI. Ser. Math., 9 (2), 2002, 239-261.

[22] D. Sanchez, Behaviour of Landau-Lifschitz Equation in a periodic thin layer, Asymptotic Analysis 41(1) (2005), 41-69.

[23] D. Sanchez, Thin layer for Landau-Lifschitz equation, preprint MAB, 2003.

[24] R. Temam, M. Ziane, Navier-Stokes equations in three-dimensional thin domains with various boundary conditions, Adv. Differential Equations, 1(4); 499-546.

[25] A. Thiaville, Extensions of the geometric solution of the two dimensional coherent magnetization rotation model, J. Magn. Magn. Mater. 182 (1998), 5-18.

[26] A. Thiaville, J.M. García, J. Miltat, Domain wall dynamics in nanowires, J. Magn. Magn. Mater. 242-245 (2002), 1061-1063. 